Portland State University

PDXScholar

\title{
The Boiling Springs Lake Metavirome: Charting the Viral Sequence-Space of an Extreme Environment Microbial Ecosystem
}

Geoffrey Scott Diemer

Portland State University

Follow this and additional works at: https://pdxscholar.library.pdx.edu/open_access_etds

Part of the Genomics Commons, and the Viruses Commons Let us know how access to this document benefits you.

\section{Recommended Citation}

Diemer, Geoffrey Scott, "The Boiling Springs Lake Metavirome: Charting the Viral Sequence-Space of an Extreme Environment Microbial Ecosystem" (2014). Dissertations and Theses. Paper 1640.

https://doi.org/10.15760/etd.1639

This Dissertation is brought to you for free and open access. It has been accepted for inclusion in Dissertations and Theses by an authorized administrator of PDXScholar. Please contact us if we can make this document more accessible: pdxscholar@pdx.edu. 
The Boiling Springs Lake Metavirome: Charting the Viral Sequence-Space of an Extreme Environment Microbial Ecosystem

$$
\text { by }
$$

\title{
Geoffrey Scott Diemer
}

A dissertation submitted in the partial fulfillment of the requirements for the degree of

\author{
Doctor of Philosophy \\ in \\ Biology
}

Dissertation Committee:

Kenneth Stedman, Chair

Valerian Dolja

Susan Masta

John Perona

Rahul Raghavan

Portland State University

2014 
(C) 2014 Geoffrey Scott Diemer 


\begin{abstract}
Viruses are the most abundant organisms on Earth, yet their collective evolutionary history, biodiversity and functional capacity is not well understood. Viral metagenomics offers a potential means of establishing a more comprehensive view of virus diversity and evolution, as vast amounts of new sequence data becomes available for comparative analysis.

Metagenomic DNA from virus-sized particles (smaller than 0.2 microns in diameter) was isolated from approximately 20 liters of sediment obtained from Boiling Springs Lake (BSL) and sequenced. BSL is a large, acidic hot-spring (with a $\mathrm{pH}$ of 2.2, and temperatures ranging from $50^{\circ} \mathrm{C}$ to $96^{\circ} \mathrm{C}$ ) located in Lassen Volcanic National Park, USA. BSL supports a purely microbial ecosystem comprised largely of Archaea and Bacteria, however, the lower temperature regions permit the growth of acid- and thermotolerant Eukarya. This distinctive feature of the BSL microbial ecosystem ensures that virus types infecting all domains of life will be present. The metagenomic sequence data was used to characterize the types of viruses present within the microbial ecosystem, to ascertain the extent of genetic diversity and novelty comprising the BSL virus assemblage, and to explore the genomic and structural modalities of virus evolution.

Metagenomic surveys of natural virus assemblages, including the survey of BSL, have revealed that the diversity within the virosphere far exceeds what has currently been determined through the detailed study of viruses that are relevant to human health and agriculture. The number of as-yet-uncharacterized virus protein families present in the
\end{abstract}


BSL assemblage was estimated by clustering analysis. Genomic context analysis of the predicted viral protein sequences in the BSL dataset indicates that most of the putative uncharacterized proteins are endemic or unique to BSL, and are largely harbored by known virus types. A comparative metagenomic analysis approach identified a set of conserved, yet uncharacterized BSL protein sequences that are commonly found in other similar and dissimilar environments.

New sequence data from metagenomic surveys of natural virus assemblages was also used to better characterize and define known virus protein families, as some of the viruses found in the BSL environment represent distant relatives of well-characterized isolates. By comparing viral genes and protein sequences from these highly divergent species, it is possible to better understand the dynamics of adaptation and evolution in the virosphere. Additionally, as structures of virus proteins continue to be experimentally determined by X-ray crystallography and cryo-electron microscopy, a merger of structural and metagenomic sequence data allows the opportunity to observe the structural dynamics underlying virus protein evolution.

Capsid (structural) proteins from two distinct Microviridae strains; a globally ubiquitous and highly sequence-diverse virus family, were identified in, and isolated from the BSL metagenomic DNA sample. These BSL capsid protein sequences, along with several other homologous sequences derived from metagenomic surveys and laboratory isolates, were mapped to the solved structure of a closely related capsid protein from the Spiroplasma phage-4 microvirus. Patterns of amino acid sequence conservation, unveiled by structure-based homology modeling analysis, revealed that the 
protein sequences within this family exhibit a remarkable level of plasticity, while remaining structurally and functionally congruent.

Lateral gene transfer is thought to have had a significant impact on the genomic evolution and adaptation of virus families. Genomic context analysis was also utilized to identify interviral gene transfer within the BSL virus assemblage. An ostensibly rare interviral gene transfer event, having transpired between single-stranded RNA and DNA virus types, was detected in the BSL metagenome. Similar genomes were subsequently detected in other ecosystems around the globe. The discovery of this new virus genome dramatically underscores the scope and importance of genetic mobility and genomic mosaicism as major forces driving the evolution of viruses.

The analyses conducted herein demonstrate the many ways in which viral metagenomic sequence data may be utilized to not only evaluate the composition of a natural virus assemblage, but to discover new viral genes, and to better understand the dynamics of both genomic and structural evolution within the virosphere. 


\section{ACKNOWLEDGEMENTS AND FUNDING}

The Boiling Springs Lake Microbial Observatory project is supported by the National Science Foundation grant MCB0702020. Samples were acquired with a research permit from the National Park Service (LAVO-2008-SCI-0030). Metagenomic sequencing was funded by the Gordon and Betty Moore Foundation through a grant to the Broad Institute. Boiling Springs Lake virus assemblage samples GAIR4 and GNX3R were sequenced at the Broad Institute.

First, I would like to extend my sincere appreciation to my advisor, Dr. Ken Stedman, and to the members of my doctoral committee; Drs. Valerian Dolja, Susan Masta, John Perona and Rahul Raghavan, for contributing their valuable time serving on this committee, and their guidance throughout the course of this research project. I would also like to thank Drs. Mike Bartlett, Radu Popa and Sherry Cady for their assistance with developing the research prospectus for the body of work presented herein.

I would also like to thank Drs. Eugene Koonin, Mart Krupovic and Arcady Mushegian for their advice and critiques of the publication: Diemer GS \& Stedman KM. A novel virus genome discovered in an extreme environment suggests recombination between unrelated groups of RNA and DNA viruses. Biology Direct. 2012, 7:13. PMID: 22515485.

Several of the findings presented herein were made possible in part by Simon Roux, through his development of the MetaVir web server, which was used to both process and analyze much of the metagenomic sequence dataset. His collegial manner and willingness to collaborate in implementing custom tools for the MetaVir program 
facilitated the often arduous process of data analysis, and his contributions in this regard are greatly appreciated. 


\section{TABLE OF CONTENTS}

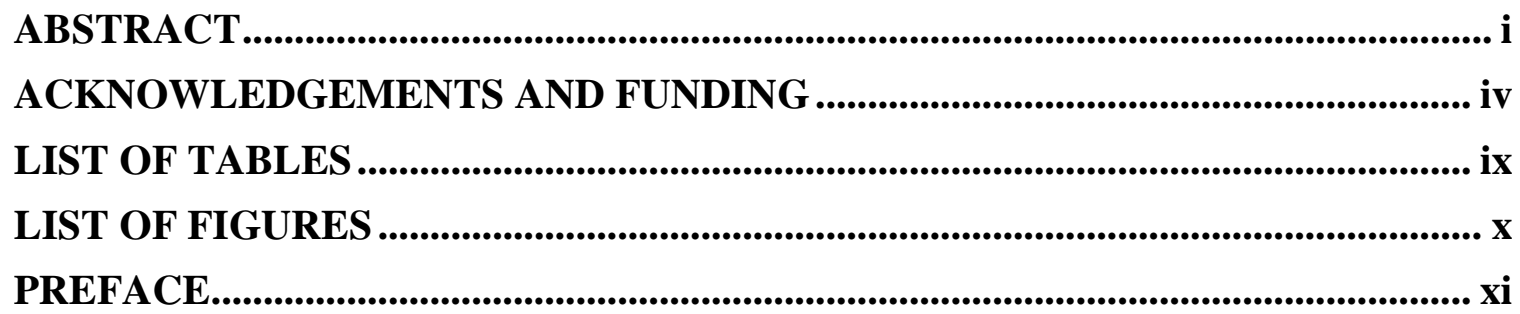

CHAPTER 1:_Comparative Metagenomics of the Boiling Springs Lake Virus

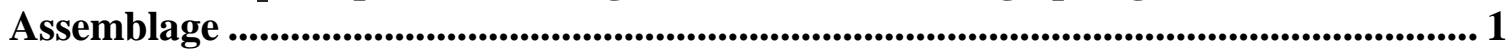

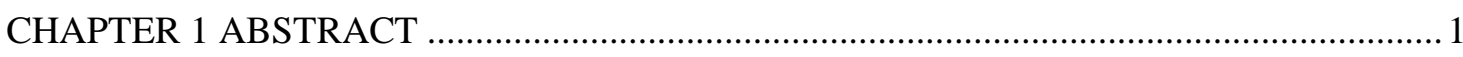

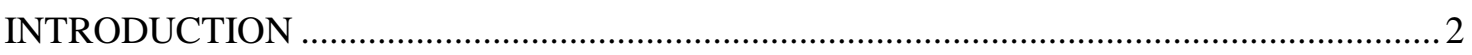

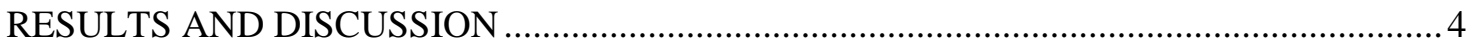

I. Boiling Springs Lake metagenomic data processing results: ........................................... 4

II. Genetic and genomic diversity in the Boiling Springs Lake virus assemblage: ................. 7

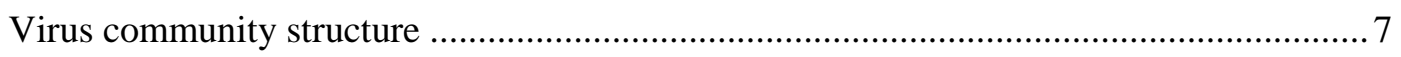

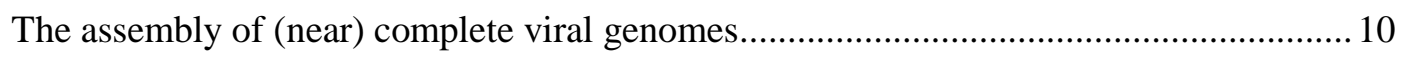

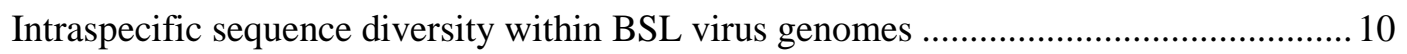

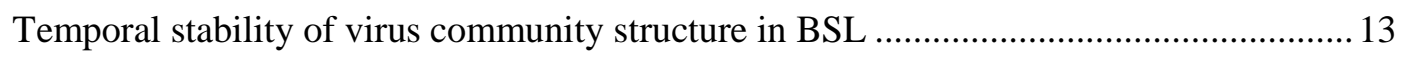

III. A contextual evaluation of BSL gene content utilizing a comparative metagenomics

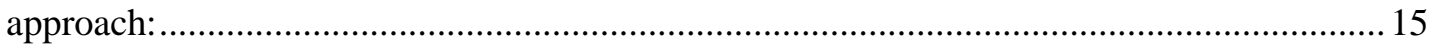

BSL metavirome sequences reflect an "extreme" environment assemblage ..................... 15

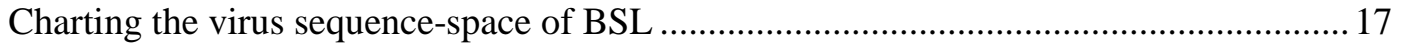

ORF rarefaction yields a more conservative estimate of virus protein families in BSL .... 19

Comparative metagenomics as a screen for identifying extreme environment-specific

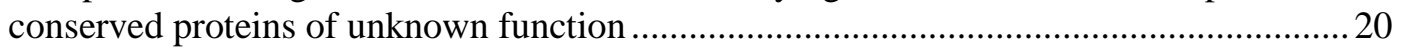

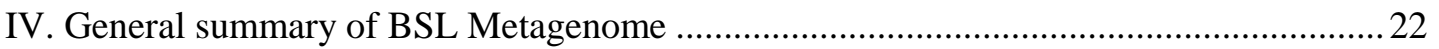

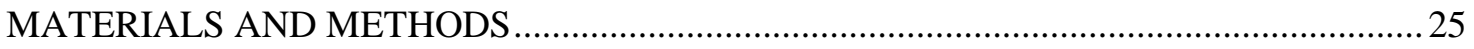

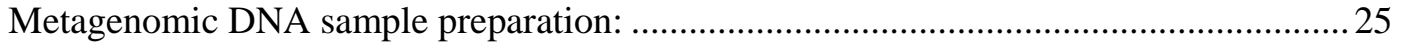

Metagenomic sequence processing, contig assembly and ORF prediction: .......................27

Taxonomic composition of the BSL virus assemblage based on sequence data: .............. 28

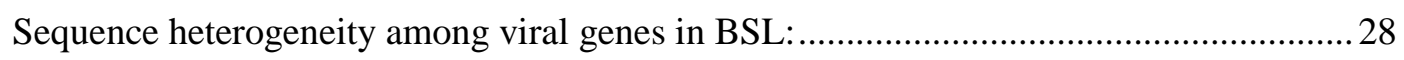

Comparative rarefaction analysis of BSL sequences and other viral metagenomes:.........29

Long-term detection of viral genes in BSL sediment samples by PCR: ...........................29

Defining ORF-types in the BSL contig dataset: ............................................................. 32 


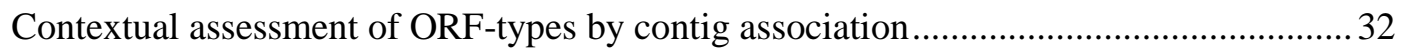

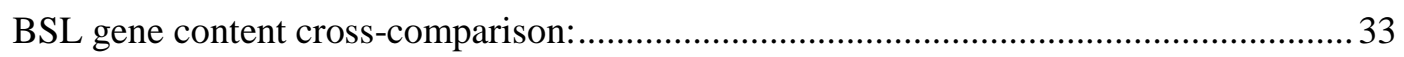

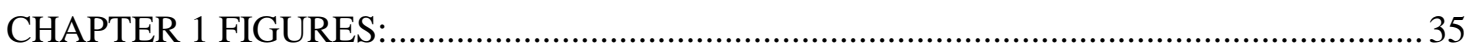

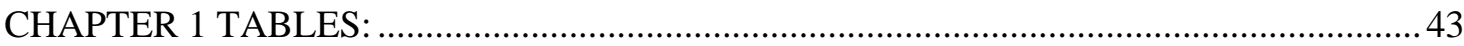

CHAPTER 2:_Utilizing Boiling Springs Lake Viral Metagenomic Sequence Data to Model the Structural Dynamics of Gokushovirinae Capsid Protein Evolution......... 45

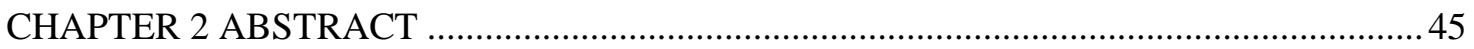

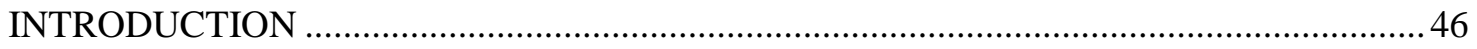

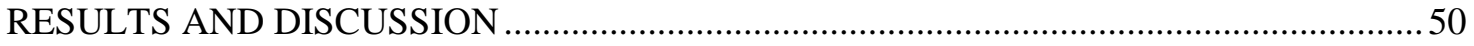

Phylogenetic assessment of the BSL microvirus major capsid proteins ...........................50

The structure of the major capsid protein and its assembly into virions .............................52

Homology modeling of Gokushovirinae, Pichovirinae and Alpavirinae capsid proteins.. 53

Identifying highly conserved regions within the microvirus major capsid protein.............55

Investigating potential capsid protein adaptations that are unique to BSL microviruses ..58

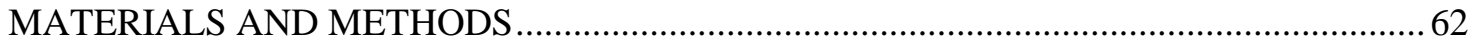

Microviridae sequences utilized in the sequence and structure analyses:.........................62

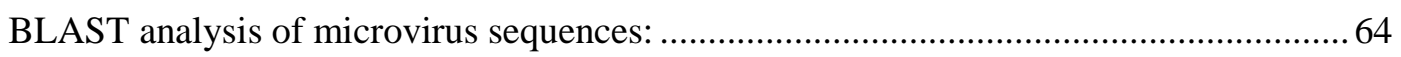

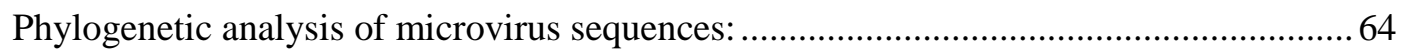

Generating structure homology models of BSL and other capsid protein sequences: ........65

Structural evaluation of the gokushovirus capsid protein monomers: ...............................66

Illustrating virion axes of symmetry and the assembly of MCPs: ....................................6 66

Generating the biological assembly file of a BSL-1436 MCP trimer showing 2-, 3- and 5-

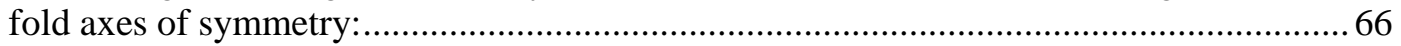

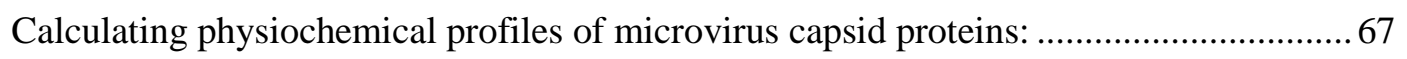

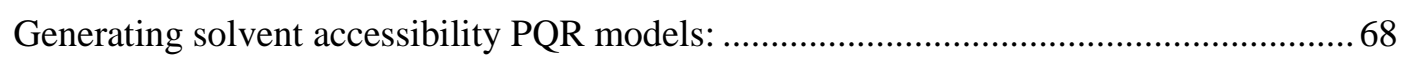

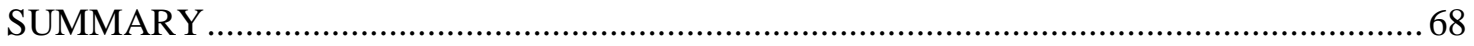

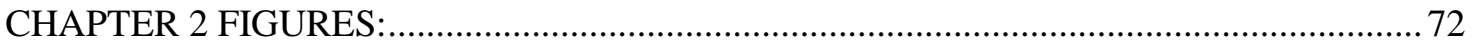

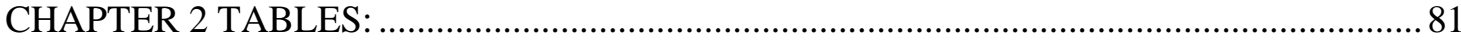

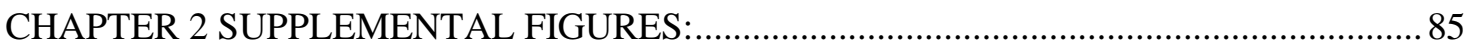

CHAPTER 3:_A novel virus genome discovered in an extreme environment suggests recombination between unrelated groups of RNA and DNA viruses ........................ 91

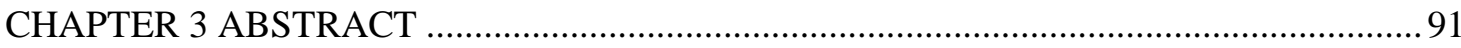

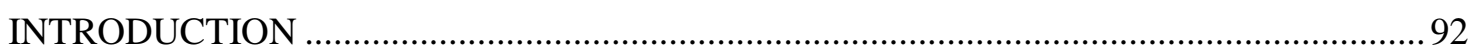




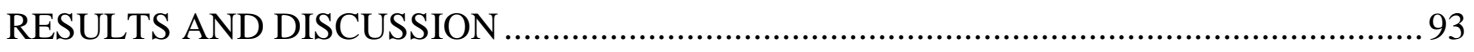

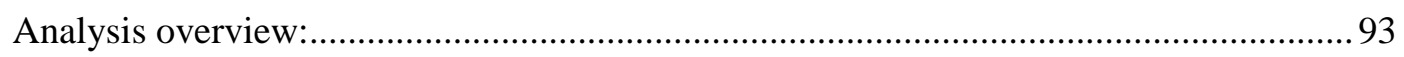

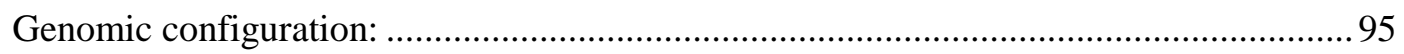

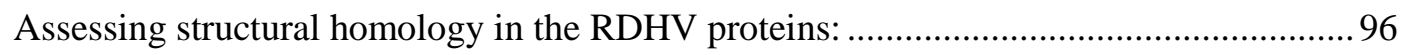

Identifying similar viruses in other environments: .......................................................... 98

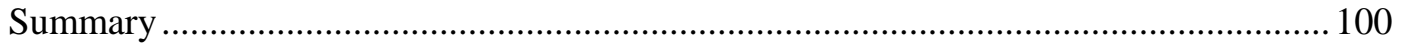

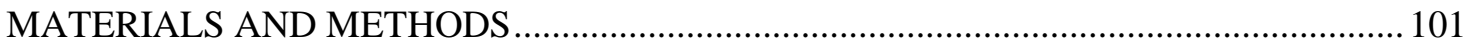

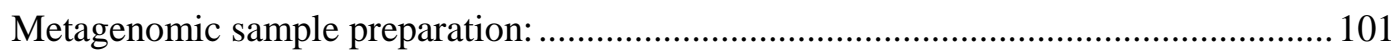

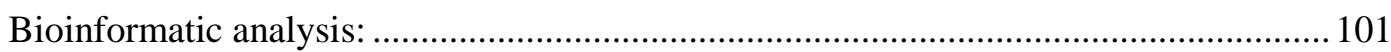

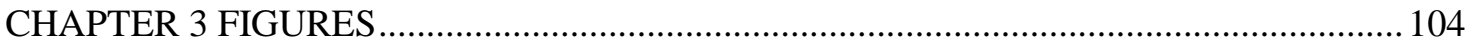

CHAPTER 4:_Synopsis and Discussion ............................................................................. 113

The advantages of sequencing low-complexity virus assemblages: .............................. 114

The BSL virus assemblage harbors few "genera", but many "species": .......................... 115

Low-complexity facilitates the charting of unknown virus sequence-space: ................... 116

Metagenomic sequence data enhances our understanding of virus protein family diversity

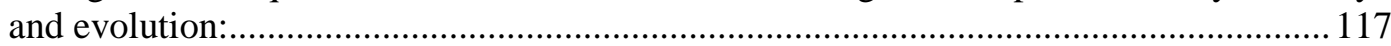

Genomic context analysis expands the potential modalities of virus evolution: ............. 119

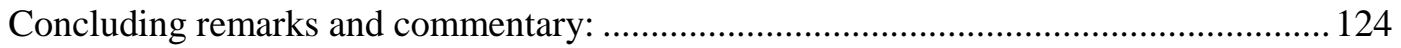

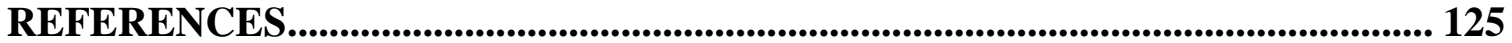

APPENDIX: Chapter 3 Copyright Information ...............................137 


\section{LIST OF TABLES}

\section{CHAPTER 1 TABLES:}

Table 1.1 Reference Metagenomes in the CAMERA Database Utilized in the BSL Comparative Analysis .................................................. 43

Table 1.2. BSL Virus Primer Information................................... 44

\section{CHAPTER 2 TABLES:}

Table 2.1 BLAST comparisons to the Spiroplasma phage-4 (SpV-4) major

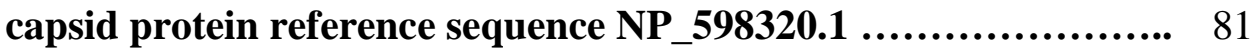

Table 2.2 Capsid protein physiochemical properties as computed by the ExPASy ProtParam tool 


\section{LIST OF FIGURES}

\section{CHAPTER 1 FIGURES:}

Figure 1.1 Predicted taxonomic composition of the BSL virus assemblage .. 35

Figure 1.2 Recruitment plot data of select virus-affiliated sequences ........ 36

Figure 1.3 Comparative rarefaction analysis of multiple metaviromes ..... 37

Figure 1.4 Long-term PCR testing for virus-like sequences in BSL ......... 38

Figure 1.5 BSL gene homologs found in other metagenomes ............... 39

Figure 1.6 ORF content of largest BSL contigs ........................... 40

Figure 1.7 Estimating the number of protein families in the BSL virus assemblage ........................................................ 41

Figure 1.8 BSL "gray matter" ORFans sorted according to environment

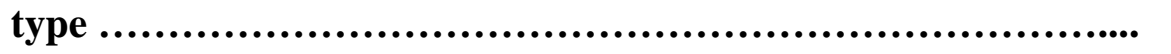

\section{CHAPTER 2 FIGURES:}

Figure 2.1 Evolutionary relationships of microvirus capsid proteins ........ 72

Figure 2.2 Homology model of the BSL-1436 capsid protein threaded to SpV-4 1KPV ...................................................... 73

Figure 2.3 Gokushovirus capsid protein assembly axes .................... 74

Figure 2.4 Structure of the complete $\mathrm{SpV-4}$ virion ......................... 75

Figure 2.5 Structural alignment of 17 Microviridae MCPs .................. 76

Figure 2.6 Spatial conservation of amino acids in CP model structures among three Microviridae clades................................. 77

Figure 2.7 Spatially conserved amino acids partially exposed on the surface of the MCP ................................................
Figure 2.8 Spatial patterns of amino acid sequence conservation in the BSL-1436 MCP assembly ................................ 79

Figure 2.9 PQR solvent accessibility mapping of capsid proteins ........... 80

Supplemental Figure S-2.1 PROMALS3D colored structure-based alignment of 17 microvirus capsid protein sequences, based on SpV-4 1KVP structure file

\section{CHAPTER 3 FIGURES}

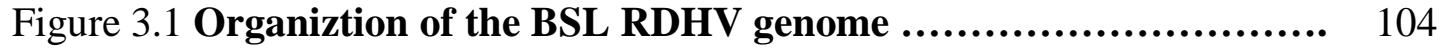

Figure 3.2 BLASTp data for Rep amino acid sequences ..................... 105

Figure 3.3 Rolling circle replicase (Rep) and capsid protein (CP) phylogenies

Figure 3.4 Rolling circle replicase (Rep) amino acid multiple sequence alignment

Figure 3.5 BLASTp data for capsid protein (CP) amino acid sequences ..... 110

Figure 3.6 Capsid protein (CP) amino acid multiple sequence alignment ... 111

Figure 3.7 BSL RDHV predicted protein structures ........................ 112 


\section{PREFACE}

The two publications listed below constitute a substantial component of the work completed during my doctoral course of study, in addition to the research findings presented in this dissertation:

- Diemer GS, Kyle JE, Stedman KM. Counting viruses using polycarbonate Track Etch ${ }^{\mathrm{TM}}$ membrane filters as an alternative to Anodisc ${ }^{\mathrm{TM}}$ membrane filters. Online Resource. Posted in July, 2012. (originally intended for publication in the journal, Limnology and Oceanography Methods) http://web.pdx.edu/ kstedman/PCTE_virus_counting_protocol.pdf

- Stedman KM, Kosmicki NR, and Diemer GS. Codon Usage Frequency of RNA Virus Genomes from High-Temperature Acidic-Environment Metagenomes. J. Virol. 2013.

CHAPTER 3 has already been published in the journal, Biology Direct (see Appendix):

- Diemer GS \& Stedman KM. A novel virus genome discovered in an extreme environment suggests recombination between unrelated groups of RNA and DNA viruses. Biology Direct. 2012, 7:13. PMID: 22515485.

This publication was chosen from all BioMed Central's 250 scientific journals as the winner of the $7^{\text {th }}$ Annual BioMed Central Research Award, and also received BioMed Central's best paper award in the "Computational and High-throughput Studies in Genomics and Systems Biology" category. 


\section{CHAPTER 1: \\ Comparative Metagenomics of the Boiling Springs Lake Virus Assemblage}

\section{CHAPTER 1 ABSTRACT}

Viral metagenomics is employed herein as a tool to evaluate the genetic and genomic diversity of viruses in an extreme environment microbial ecosystem. The diversity of viral gene content, including novel and undefined genes, is characterized using a comparative metagenomics approach. The Boiling Springs Lake virus assemblage, based on taxonomic assignment of sequences that are similar to known virus genes, appears to be comprised of a relatively small number of virus families or groups. An unexpectedly low complexity of the sample resulted in a greater depth of sequencing than was anticipated, which revealed extensive sequence heterogeneity within many of the predominant putative virus groups. The most prevalent and closely related homologous sequences shared with other metagenomic surveys are found in sites with similar physiochemical profiles, however, the novel gene content comprising the virus assemblage is extensive and largely endemic to this particular biome. Most, if not all, viral metagenomes or "metaviromes" are comprised largely of unidentifiable sequences, which are sometimes presumed to represent as-yet-undiscovered viral taxa. However, a substantial component of the unknown gene content in this extreme-environment virus community appears to be harbored by known virus groups, and to a lesser extent, is comprised of conserved proteins of unknown function that are found in other biomes. 
Comparative metagenomic analysis identifies common, conserved unknown protein families as a target for future research and bioprospecting efforts.

\section{INTRODUCTION}

Boiling Springs Lake (BSL) is an acidic, fumarole-heated lake located in Lassen Volcanic National Park, USA [1, 2]. This highly oligotrophic hot spring environment maintains a consistent minimum temperature of approximately $52+/-3^{\circ} \mathrm{C}$ with a $\mathrm{pH}$ of approximately 2.2 throughout, however regions in the vicinity of exposed and shallow fumaroles reach as high as $93^{\circ} \mathrm{C}$ [2]. The temperature throughout the majority of the water column and shallow sediments allows a small consortium of acid- and thermotolerant eukaryal protists [3] to co-exist with thermoacidophilic Archaea and Bacteria originating from the high $\left(73-83^{\circ} \mathrm{C}\right)$ and extreme temperature regions $\left(93^{\circ} \mathrm{C}\right)$ of the lake $[2,4]$. Upwelling from a series of submerged fumaroles results in a thorough mixing of the water column, as well as the convective dispersal of microbial organisms and fine sediment particles [2]. The lake itself is also believed to be quite young geologically. While BSL is known to predate 1864 [5], and is considered to be relatively stable, similar geothermal environments are rapidly created and destroyed on a geologic timescale. For example, Frying Pan Lake, located in Waimangu Volcanic Rift Valley, New Zealand is a geothermal lake quite similar to BSL that is known to have formed in 1918 [6].

Viruses play vital ecological roles in microbial ecosystems, and are often the key predator and driver of nutrient cycling, particularly in oligotrophic environments [7-9]. 
Viruses are also thought to play a part in ecosystem-wide adaptation processes by providing, and possibly generating, novel genes that are shuttled among host organisms [10-15]. Moreover, virus-mediated gene transfer is thought to have been particularly relevant in early-earth microbial ecosystems and perhaps even crucial for the emergence of autonomous cellular life forms [16-18]. The effect viruses have on the adaptation and evolution of purely microbial ecosystems, particularly in geologically young environments like BSL, however, remains unknown.

The number of virus-like particles (VLPs) in the BSL water column, like other high-temperature, acidic environments $[19,20]$, is too low to be accurately calculated with current methods. However there are approximately $1 \times 10^{6}$ VLPs per cubic centimeter of sediment, as determined by epifluorescent microscopy [21]. A metagenomic survey of virus-sized particles extracted from BSL sediment was conducted to better understand the role viruses play in this highly oligotrophic and purely microbial ecosystem.

Viral metagenomic data is first utilized in this study to identify virus families present in the system, to characterize the uniqueness of the BSL virus community, and to ascertain the overall level of sequence divergence between identifiable viral genes in BSL and their counterparts in other annotated sequence databases. A low diversity of viruses in the BSL sample fortuitously resulted in a greater depth of sequencing than was anticipated, which allowed many large contigs to be assembled at high stringency. It was, therefore, possible to predict many large and complete open reading frames (ORFs) within the set of contigs, and to conservatively estimate the diversity of viral protein families present in BSL. 
A common feature of viral metagenomes is that a large majority of the sequence reads (and ORFs) have no identifiable homolog in public databases [22-25]. Unfortunately, this extensive reservoir of unknown sequence-space is frequently ignored when the community structure of uncultivated viruses is analyzed. Conserved proteins of unknown function were identified by cross-comparing BSL ORFs to other metagenomic datasets. These common, conserved ORFs of unknown function, as well as completely novel putative ORFs unique to BSL, could then be evaluated contextually on the basis of their co-localization with other genes within large contigs containing two or more ORFs. Cross-comparison of BSL to other metagenomic datasets was also done as the basis for evaluating whether the virus community structure and genes of viral origin in BSL are biome-specific, or similar to what has been found in other extreme environments.

\section{RESULTS AND DISCUSSION}

\section{Boiling Springs Lake metagenomic data processing results:}

The BSLM $\phi 29$ amplified DNA was shown to contain only trace amounts of microbial DNA, exhibiting roughly 50,000 times less $16 \mathrm{~S}$ rRNA gene content than E. coli genomic DNA by weight based on 16s rRNA PCR, prior to sequencing. Contig assembly of the 390,208 2009 BSLM pyrosequencing reads using Newbler v.2.0 (Roche 454 sequencing) was carried out following quality-filtering using the GenomeQuest (www.genomequest.com/) read processing workflow. This resulted in the assembly of 
$83.6 \%$ of the reads, with $6 \%$ of the unassembled reads designated as "singletons". The remaining $10.4 \%$ were designated as "repeats", "outliers" and "too short", and were removed. The average read length was 320 bases, and the longest unassembled singleton is 1,022 bases. The assembly resulted in the formation of 4,640 contigs, with a maximum size of 15,046 bases, however, only $15 \%$ of the contigs were longer than 500 bases. A set of 925 contigs and singleton reads longer than 500 bases was manually compiled and uploaded to MetaVir (MetaVir ${ }^{2}$-Beta) [26] for open reading frame (ORF) prediction. This process predicted 2,073 whole and partial ORFs from the 925 sequences longer than 500 bases. The average ORF size is 107 amino acids (AA), with a minimum of 19AA, a maximum of 955AA, and a median value of 81AA. Partial ORFs account for $50 \%$ of the total ORFs predicted. Forty percent of all ORFs are larger than 100AA, and 78\% are larger than 50AA in length. Even though the majority of the complete and partial ORFs predicted are relatively small, and not ideal for accurately estimating whether an ORF is actually expressed as a protein, sequence similarity observed within the commonlyoccurring ORFs and ORF fragments was utilized as a means of determining cluster-based patterns of genetic heterogeneity within the virus assemblage as a whole.

PHACCS analysis [27] (http://phage.sdsu.edu/phaccs) calculated the presence of 90 genotypes in the sample, with an evenness score of 0.88 (on a 0 to 1 scale) with the most abundant genotype constituting $13.3 \%$ of the predicted 90 genotypes. The ShannonWiener Index score of 3.95, also calculated by PHACCS, indicates a fairly even distribution of genotypes within the sample. 
Rarefaction of the 390,208 total BSLM reads in MetaVir resulted in the formation of 163,368 clusters at $98 \%$ stringency, 62,153 clusters formed at $90 \%$, and 27,019 clusters formed at 75\% stringency. The calculated read-per-cluster ratios are thus; 2.4, 6.3 and 14.4 reads-per-cluster, respectively.

The taxonomic composition tool in MetaVir ${ }^{2}$-Beta revealed whether an individual ORF is identifiable as a known viral gene, based on tBLASTn comparison to the MetaVir virus sequence database. This search, conducted in November of 2012, found that 97 of the 2,073 predicted ORFs matched a sequence in the virus sequence database used by MetaVir at the time. The unaffiliated (presumably non-viral) BSL ORF sequences were then uploaded to the CAMERA-2.0 portal [28] (http://camera.calit2.net) and queried against the CAMERA virus sequence database using the "BLAST workflow" tool. This search identified 44 additional ORFs that were not originally characterized by MetaVir as viral. The unaffiliated ORFs were also searched against the CAMERA reference sequence database to identify possible cellular or "host-associated" genes. This search yielded a total of 27 putative host-associated genes. The remaining 1,905 unknown ORF were considered putative "dark matter" [29] ORFs or "ORFans" [30]. To further refine this ORFan dataset, putative dark matter ORFans were searched against 12 metagenomes (summarized in Table 1.1) in the CAMERA database using tBLASTn applying a $10^{-3} \mathrm{E}-$ value cutoff. This search yielded 61 hits to unannotated sequences found in other environmental metavirome surveys. These common, conserved hypothetical proteins are herein designated as "gray matter" ORFans. The resulting relative abundance of nonredundant, non-clustered metavirome ORFs is; 97 (4.7\%) MetaVir virus database, 44 
(2.1\%) CAMERA virus database, 27 (1.3\%) CAMERA reference sequence database, or "host-associated" ORFs, 61 (2.9\%) gray matter ORFans and 1844 (89\%) dark matter ORFans.

\section{Genetic and genomic diversity in the Boiling Springs Lake virus assemblage:}

Virus community structure

Based on automated taxonomic composition assessment using MetaVir, ORFs found to be similar to those of known single-stranded and double-stranded DNA virus families were detected in the Boiling Springs Lake (BSL) metavirome (Figure 1.1). The putative virus groups detected mirror the diversity of microbial host genera found in the BSL microbial ecosystem $[3,4]$, such that there appear to be a relatively large number of archaeal viruses and bacteriophage, and a small number of virus groups that infect eukaryotes. Eukaryal organisms found in the lower temperature zones of Boiling Springs Lake include several Viridiplantae (green algae) species, Rhodophyta (mainly the red algae Cyanidium and Cyanidioschyzon), Stramenopiles and Alveolata (diatoms, flagellates and ciliates), Opisthokonts (mainly fungi) and the Euamoebida (Hartmannella, Echinamoeba and Acanthamoeba species) $[3,31]$ Of the viruses known to infect eukaryotes, sequence homologs from algal and amoebal viruses predominate in BSL.

The identification of sequences similar to common virus families in the BSL virus assemblage using automated "best-hit" taxonomic composition tools featured by MetaVir and MG-RAST [32] (MG-RAST 2.0 generated similar taxonomic composition data that 
are not shown as part of this analysis) do not indicate how similar the metagenomic sequences are relative to reference genomes, or indicate the number of distinct genes detected per virus reference genome. Recruitment plot data from MetaVir was therefore utilized to illustrate the overall extent of divergence between viral protein sequences isolated from BSL and homologous sequences from known cultured isolates. In many instances, only a small number of BSL reads will actually map to a given reference genome, or alternatively, many reads will canvas only small, isolated regions of a given reference genome. Recruitment plot figures published by Schoenfeld et. al. [33] from circum-neutral hot springs are very similar to those produced from BSL data, in that large reference genomes containing tens or hundreds of genes recruit reads matching very small regions, and often canvas as few as one or two genes within each reference genome.

Reference sequences from twelve known virus groups, spanning all host domains, were used to generate recruitment plots of BSL metavirome sequence data using MetaVir. The quality of the sequence match between a given BSL sequence and the cognate reference sequence based on the tBLASTx E-value was plotted with the total number of BSL reads recruited and coverage of the mapped reference viral genome sequence. These data indicate that most of the BSL virus genomes are quite distinct by comparison to the reference sequences (Figure 1.2). Not only is there exceedingly low coverage of the reference genome in most cases, such that only one or two reference genes per genome recruit homologous BSL reads (plot data not shown), but only 5 out of the 16 regions mapped by BSL reads are greater than $50 \%$ identical to the reference 
sequence at the amino acid level. While taxonomic composition data imply that several well-characterized virus families are represented in BSL, upon more detailed inspection, very few BSL viruses identified using this tool actually appear to be closely related, on a genetic or genomic level, to any other known virus.

One notable example from the BSL DNA metavirome data illustrates the inherent shortcomings of short read-based taxonomic composition analysis. A capsid proteinencoding gene within a $c a .4 .3 \mathrm{~kb}$ contig was detected in the BSL assembly that demonstrated sequence-similarity to the capsid protein gene of the Tombusviridae group of linear (+)ssRNA plant viruses and non-classified ssRNA fungal viruses. A detailed analysis later revealed that this ssRNA virus capsid protein-like gene was located within a small, circular DNA genome encoding a replication initiation protein (Rep) gene similar to those found in the Circoviridae. The ssRNA virus capsid protein gene was most likely acquired by the circovirus-like genome, possibly by interviral RNA-DNA recombination, and has hence been provisionally dubbed an "RNA-DNA Hybrid Virus" (RDHV) [34]. Several other examples of this novel group of virus genomes have since been detected in other microbial and viral metagenomic datasets [35], including reclaimed water in Florida [36, 37], Korean air [38], deep sea sediments in the Pacific [39] and off the coast of Greenland [40], as well as freshwater lake systems in New York state [41], Antarctica [42] and France [35] (see CHAPTER 3 for detailed analysis). 
The assembly of (near) complete viral genomes

Four complete genomes of small, circular DNA virus genomes have been identified in the set of assembled BSL contigs (all complete genomes described within this section will be submitted to GenBank). All four genomes have been subsequently amplified by inverse PCR from the 2009 BSLM DNA sample. One of these is a 1,551nucleotide genome encoding a putative 244 amino acid capsid protein (CP) ORF yielding a top BLASTp E-value of $4 \times 10^{-6}$ by comparison to an uncultured marine virus, and $3 \times 10^{-5}$ by comparison to a Diporeia species-associated circular DNA virus [43]. The other putative 193 amino acid ORF matches a bat circovirus-like replication initiation protein (Rep) with an E-value of 9x10-9. Another small circular virus, 1,977 nucleotides in length, encodes only one identifiable 284 amino acid ORF, yielding a top BLASTp Evalue of $2 \times 10^{-63}$ by comparison to a circovirus-like Rep from an uncultured marine virus, however other searches match geminivirus-associated alphasatellite Rep sequences. Additionally, two distinct gokushovirus genomes within the Microviridae group have been identified in the contig dataset. These gokushoviruses exhibit gene content and synteny mirroring the well-characterized chlamydiaphage and other environmental isolates from marine and freshwater sources [44, 45] (see CHAPTER 2 for detailed analysis).

Intraspecific sequence diversity within BSL virus genomes

Multiple amino acid sequence variants of viral genes appear to be commonplace in the BSL metavirome. For example, six different contigs harbor archaeal Halorubrum 
pleomorphic virus (HRPV)-1-like Rep protein ORFs. HRPVs infect the halophilic archaeon, Halorubrum, and have large (in the 7 to $10 \mathrm{~kb}$ range), circular, ssDNA genomes [46-48]. Several attempts to amplify a complete BSL HRPV-like genome by inverse PCR have been unsuccessful. However, all attempts at amplifying segments of the HRPV-like sequence from BSL DNA have succeeded, implying that the BSL HRPVlike virus genomes are not circular. As BSL is not a hypersaline environment, the BSL moieties probably represent a distant relative of HRPV that infects a non-halophilic archaeon, or a novel virus type that acquired an HRPV-like Rep through horizontal gene transfer. All six HRPV-like contigs, while not identical, are very similar in terms of their gene content and synteny, signifying the presence of a diverse population of novel HRPV-like viruses in the BSL virus assemblage.

In addition to the genotypic variation found in the aforementioned BSL gokushovirus sequences and the HRPV-1-like contigs, five distinct contigs in the BSL metavirome contain sequences similar to the Acidianus filamentous virus (AFV) DNA binding protein sequence.

This common theme illustrates another remarkable attribute of the BSL metavirome, and possibly of the BSL viral community itself: While a low diversity of virus genera or groups is observed within BSL according to PHACCS assessment and taxonomic composition assignment (Figure 1.1), there is an unexpectedly high degree of sequence heterogeneity within these groups, in that some of the viruses comprising the assemblage appear to be represented by several distinct genotypes. This pattern has also been observed in metagenomic surveys of circum-neutral hot spring environments by 
Schoenfeld et. al. [33]. In that case, however, the pattern of genotypic heterogeneity was extrapolated based on the observation that loosening the stringency settings of the assembly to $50 \%$ identity increased the formation of long contigs. Conversely, BSL metagenomic reads could readily be assembled at very high stringency (98\% identity spanning 20 nucleotides) into large contigs, and intraspecific diversity was not extrapolated in this case, but observed directly through the alignment of homologous contigs and ORFs.

To determine whether this pattern of low $\alpha$-diversity coupled with high intraspecific sequence diversity is unique to BSL or whether it is common in other environments, rarefaction curves were generated for the BSL metavirome and several other published viral metagenomes available for analysis in MetaVir. These metagenomes were selected to represent a broad range of physiochemical environments and ecotypes (Figure 1.3). The ratio of sequence reads per number of clusters formed by rarefaction illustrates contrasting patterns of species richness and intraspecific heterogeneity among virus assemblages isolated from different environments. Rarefaction has the most pronounced effect on the BSL metagenomic reads when compared to other viral metagenomes analyzed, in that the read-per-cluster ratio is inversely proportional to rarefaction stringency. The BSL metagenome has the smallest proportion of unclustered reads and the largest proportion of reads in low stringency clusters.

If the sample is either low-diversity or thoroughly sequenced, clusters formed at low stringency (75\%) are expected to be composed of a larger number of reads, on 
average, than would be formed at high sequence stringency (98\%). By contrast, most of the sequence reads from highly diverse virus assemblages or under-sampled metagenomes are unique and will not be binned into homologous clusters, thus at both high and low stringencies the read-per-cluster ratios will be close to unity (see GOS MOVE-858 dataset in Figure 1.3). While there is no clear relationship in rarefaction clustering patterns between metaviromes with respect to physical environment, the most similar rarefaction patterns to BSL appear to be derived from oligotrophic environments. Low $\alpha$-diversity coupled with high intraspecific or strain diversity may, therefore, be a pattern that depends more on the trophic state of the system than on physiochemical parameters, however an extensive analysis of oligotrophic systems would be required to substantiate this hypothesis.

\section{Temporal stability of virus community structure in BSL}

Several of the virus sequences identified in the 2009 metagenomic sequence data were detected by PCR in samples collected from BSL sediment in 2010, 2011 and 2012 (Figure 1.4). The only viral sequence detected every year in BSL since 2009 is a novel archaeal Acidianus two-tailed virus (ATV)-like sequence. Moreover, the ATV sequence is the only one detected in DNA isolated from the high-temperature $\left(93^{\circ} \mathrm{C}\right)$ region of the lake. This is consistent with expected results, as ATV infects the hyperthermophilic archaeon, Acidianus [20] however, the host for the BSL ATV-like virus remains unidentified. Ribosomal RNA sequences corresponding to hyperthermophilic Archaea have been found in both the hot $\left(93^{\circ} \mathrm{C}\right)$ and cooler $\left(53^{\circ} \mathrm{C}\right)$ regions of the lake $[2,4]$, and 
efforts to cultivate archaeal species from BSL in vitro are currently underway, with the intent of isolating viruses identified from the metagenomic data. The second most frequently detected sequence throughout the 4-year sampling period comes from a Halorubrum pleomorphic virus (HRPV)-like sequence. The HPRV-like genome, which may or may not be single stranded in the virion, has also been detected in 2010 and 2012 . Small, circular single-stranded (ss) DNA viruses detected in the original 2009 metagenomic sequence data have not been detectable by PCR in samples collected in subsequent years.

Sequences related to the giant algal Phycodnaviridae (ATCV and OtV) and amoebal Mimiviridae (APMV and MGVC) virus genes have also been detected in the BSL sequence dataset, despite the fact that the metagenomic DNA was extracted from particles smaller than 200 nanometers in diameter. One of the genes detected encodes the enzyme GDP-D-mannose 4,6 dehydratase. This gene is common to both virus and host and is located immediately downstream of another host-associated glycosyltransferase gene. Moreover, these two genes are found in the algae-infecting Ostreococcus taurii Virus-2 (OtV-2), and are similarly co-localized within the same region of the putative BSL viral genome.

Surprisingly, no virus sequences were detected in samples from 2011 from site-A. One explanation for this lack of data may be attributable to the different manner in which samples from that year were prepared (see Methods section). In 2011, virus-sized particles were extracted from site-A and site-D sediment samples using a filter-based extraction technique instead of by tangential flow filtration (TFF), as was used for the 
2009, 2010 and 2012 samples. While DNA was recovered using the filter-based method, it is possible that the DNA is derived largely from microbial sources, or that few virus particles were actually retained by the filter prior to DNA extraction.

Unfortunately there have been relatively few long-term studies on environmental viral ecosystems. The 4-year PCR study (Figure 1.4) indicates that sporadic changes in the virus assemblage may be common, and this pattern has been observed by others [ 42 , 49-52]. Small circular ssDNA viruses are known to have relatively high mutation rates [53] thus, it is not surprising that detection of sequences from these virus types has not been possible since the metagenomic sample was collected in 2009. Conversely, the large, double-stranded DNA bacteriophages (Caudovirales), amoebal and algal viruses (Mimivirus and Phycodnavirus) and archaeal viruses (SSV-K/ ASV-1, AFV, ATV), appear to be relatively stable within the BSL ecosystem (Figure 1.4).

\section{A contextual evaluation of BSL gene content utilizing a comparative metagenomics approach:}

BSL metavirome sequences reflect an "extreme" environment assemblage

A comparative metagenomics approach was used to address the assumption that certain environments will select for particular viruses and viral genes [11, 54], by comparing a set of 229 virus, host-associated and gray matter open reading frames from the BSL metavirome contigs against a collection of publically available metagenomes in CAMERA in which BSL-like sequences were found by tBLASTn searches. Putative 
genes from BSL producing tBLASTn E-values smaller than $1 \times 10^{-3}$ were plotted according to the other metagenome in which a matching sequence is found, in an $\mathrm{X}-\mathrm{Y}$ bubble plot. The diameter of the bubble is proportional to the E-value of the sequence match between the BSL and associated metagenome (Figure 1.5).

Sequences homologs to BSL sequences are found in both similar and dissimilar environments, however there are generally more BSL ORF homologs in "extreme" and freshwater environments than are present in marine environments. Moreover, the most similar sequences (by tBLASTn) are found in a variety of "extreme" hypersaline, acidic or high-temperature environments. As the physiochemical nature of these extremes are each quite distinct, it is difficult to account for sequence commonalities observed between these otherwise dissimilar sites without a detailed cross-comparison of the host organisms present in each system. This analysis also indicates that while BSL contains sequences found in extreme, freshwater and even marine samples, there is a lesser degree of overlap among ORFs found within particular environments, as well as a lesser degree of sequence similarity between differing physiochemical environment types. Overall, the viral gene content of BSL most closely resembles that found in other "extreme" environments; namely acid mine drainage, hypersaline and hydrothermal sites, and qualitatively supports the premise that virome gene content is biome-specific. $[11,25,33$, 55, 56] (Figure 1.5).

The functional capacity and the extent of genetic diversity within the virosphere as a whole has not yet been quantitatively defined $[57,58]$. Taxonomic assignment of viral metagenomic sequences generally indicates that upwards of $90 \%$ of the sequence 
reads in any given dataset are not identifiable when compared to annotated sequence databases $[11,23-25,57,59,60]$. This vast, unknown component of viral metagenomes has been appropriately dubbed the "dark matter of sequence-space" [29], and is often disregarded during metagenomic characterization of a virus assemblage. However, with great strides being made in the development of bioinformatics pipelines [61], and as substantial contributions of metagenomic survey data are deposited into public sequence databases, it is now possible to begin charting this unknown sequence terrain.

The low diversity of viruses in the BSL sample resulted in a thorough sequencing of the metagenome which, in turn, allowed large contigs to be assembled at high stringency. Thus, it was possible to predict open reading frames with high confidence. Not only could some ORFs be identified as viral genes based on sequence database comparisons (Figure 1.2), some of the unknown ORFs could be characterized as conserved proteins of unknown function based on whether homologs were present in other metagenomic datasets. Rarefaction analysis was also performed on the entire set of ORFs in order to produce a more conservative estimate of viral protein family diversity in BSL. The abundance of relatively large contigs allowed these ORFs to be analyzed contextually with respect to their placement within each contig, and according to their colocalization with other identifiable viral genes (Figure 1.6).

\section{Charting the virus sequence-space of BSL}

Sequence reads from the BSL metavirome were assembled into contigs, and the ORFs were classified as being either an "affiliated" (i.e., identifiable) viral gene or an 
"unaffiliated" (i.e., non-viral) gene by comparison to MetaVir, CAMERA and GenBank databases. Unidentifiable "dark matter" ORFs are commonly referred to as "ORFans" [30]. The identification of common yet unidentified, conserved BSL ORFans was carried out by cross-comparing the BSL "dark matter" ORFans to other publically available metagenomic and metavirome sequence databases using tBLASTn. Any homolog of a BSL metavirome ORFan identified in another metagenomic dataset is referred to herein as a "gray matter" ORFan. This process identified 3\% of the 2,073 BSL metavirome ORFs as being "gray matter" ORFans.

The BSL virus and host-associated ORFs, in addition to the dark matter and gray matter ORFans, were mapped to their contig of origin in order to provide a genomic context for assessing the distribution of ORF types. Strikingly, many of the dark and gray matter ORFans are located in contigs containing virus-affiliated gene homologs, indicating that much of the "dark matter of sequence space" (at least in BSL) is harbored by contigs also containing ORFs similar to those found in known virus families (Figure 1.6). Based on the 7 largest BSL contigs, each containing greater than ten ORFs, $24 \%$ of all ORFs are affiliated with known viruses and $71 \%$ are ORFans (61\% dark matter and $10 \%$ gray matter). This conservatively suggests that the majority of all predicted genes in BSL virus assemblage are unique, and roughly an additional ten percent of BSL genes are commonly found in other environments but are as yet uncharacterized. Even though 3 out of the 7 largest BSL contigs are comprised entirely of ORFans, each possibly representing novel taxa, the co-localization of known viral genes and ORFans in the remaining 4 contigs suggests that the viruses in BSL are largely comprised of ORFans. 
These results very closely resemble the findings of Pedulla et. al. [29], which indicate that the majority of genes found in a survey of 10 novel mycobacteriophage genomes were ORFans. Similar findings have been recently reported in metagenomic contigs derived from marine virus assemblages that have been fractionated to reduce the complexity of the viruses sampled prior to metagenomic sequencing [62]. Contextual analyses such as these underscore the importance of sequencing low-diversity or lowcomplexity assemblages, as the sequence data produced is more readily assembled into large contigs, which in turn allows for a much more thorough analysis than is possible for highly complex metaviromes. Thus, it appears that the "dark matter" filling the virosphere is largely harbored by known virus groups, and not necessarily indicative of there being vast numbers of novel virus "species" in the environment that are yet to be discovered $[25,58,63]$.

ORF rarefaction yields a more conservative estimate of virus protein families in $B S L$

The BSL ORF categories were also clustered by rarefaction at $75 \%$ amino acid sequence identity in order to determine the relative abundance of unique protein families within each category, thus generating a more conservative estimate of genetic novelty in BSL [22]. Clustering each ORF category independently indicates that the percentage of novel dark matter ORFans in BSL is substantially lower than is indicated prior to rarefaction (from $89 \%$ to $78 \%$ ), whereas gray matter ORFans and virus- and hostassociated genes are not as substantially reduced (Figure 1.7). While greatly reducing the total number of putative proteins comprising by the virus assemblage, the relative 
percentages of each subset are not significantly altered, in that the dark matter ORFans (possibly unique to the BSL virus assemblage) still predominate. The high proportion of true dark matter ORFans, relative to the gray matter ORFans, illustrates the extraordinary degree of genetic novelty contained specifically within the BSL viral ecosystem, and underscores the role viral genetic novelty undoubtedly plays in the adaptation and community structure of both viral and host communities.

Comparative metagenomics as a screen for identifying extreme environment-specific conserved proteins of unknown function

One rationale for conducting metagenomic surveys of extreme-environment virus assemblages is the search of novel thermostable enzymes that might prove useful as molecular tools [64]. By first identifying gray matter ORFans using a comparative metagenomics approach, each of these ORFs may be further analyzed individually with respect to the physiochemical environment in which they are most commonly found. The identification of conserved unknown proteins common to high temperature environments, for example, would allow a researcher to conduct an efficient search for novel thermostable proteins in silico, before any coding sequences are cloned, expressed and assayed for a given function.

A Cytoscape network diagram showing the distribution of gray matter ORFans, constructed from Venn analysis data, maps homologs of the common, conserved unknown BSL protein sequences (i.e., gray matter ORFans) to other environments (Figure 1.8). Of the 61 non-redundant BSL gray matter ORFans, 23 are found in multiple 
locations and 38 are found only in one other location. Interestingly, a majority of the commonly occurring gray matter ORFans are in reclaimed and freshwater samples; many of which are also present in multiple sites. Many of the gray matter ORFans in BSL are found in highly acidic mine drainage and circum-neutral terrestrial hot spring environments, whereas very few are found in hypersaline and hydrothermal extreme environments. In contrast to ORFans found in reclaimed and freshwater samples, the acidic and terrestrial hot spring sites contain only three BSL ORFan homologs that are shared among multiple environments, whereas most are found in only one other site besides BSL. This may indicate that many of these genes are specifically adapted to, or selected by physiochemical factors in the environment. Unexpectedly, very few BSL gray matter ORFans are found in hypersaline and hydrothermal environments, despite the fact that many virus-affiliated ORF homologs are found in these sites (Figures 1.5 and 1.8). Moreover, the degree of sequence similarity is higher overall between BSL and the terrestrial hot spring, reclaimed and freshwater sequences, and much less so in acidic or marine environments. Until these gray matter genes can be isolated from the environment and expressed for functional analysis, it will be difficult to completely explain their observed distribution patterns. However, using a comparative approach to identify gray matter ORFans could help expedite the search for novel proteins that may be useful as molecular tools or in nanotechnology applications $[64,65]$. 


\section{General summary of BSL Metagenome}

Coarse taxonomic assessment of the BSL viral metagenome indicates that many of the virus groups one might expect to find in an extreme environment microbial ecosystem, composed of host organisms from all domains of life, are present within the assemblage (Figure 1.1).

Low-complexity data allowed for large contigs to be assembled at high stringency, and for either complete or large open reading frames to be predicted and mapped to their contig of origin. Doing so provided a contextual basis for determining the genomic and genotypic similarity between metagenomic sequence data and a cognate virus reference genome (Figure 1.6). When ORFs are predicted from high-stringency contig assemblies, rarefaction or protein clustering assessment yields a more realistic estimation of genetic diversity and intraspecific variety possessed by a natural virus assemblage than is possible from fragmentary sequence reads (Figures 1.3 and 1.7). Comparative rarefaction of multiple metagenomic datasets also suggests that while $\alpha$ diversity may primarily be a function of the trophic state index of an environment, such that oligotrophic sites appear to sustain lower levels of virus subgroup diversity, it is also possible to detect a high level of intraspecific diversity in these subgroups.

The long-term PCR detection of viral metagenomic sequences over a four year period in BSL indicates that large, double-stranded DNA viruses and bacteriophage are the most stable over time, whereas natural fluctuations in the population, or possibly 
higher mutation rates in small, single-stranded DNA viruses, makes them more difficult to detect over extended periods of time (Figure 1.4).

Comparative metagenomic analysis of ORF sequences spanning multiple environments demonstrates that the gene content, or the types of viruses present in a given environment appear to be determined by physiochemical factors (Figures $\mathbf{1 . 5}$ and 1.8). For example, particular sets of ORFs are common to similar environments, and these sets vary in composition and sequence-similarity according to differing environmental conditions. Not only do some of the genes found in BSL exhibit a higher degree of sequence similarity to those found in similar extreme and freshwater environments, there are also a higher number of them, relative to what is found in marine environments (Figure 1.5).

While trophic state index appears to influence the level of virus subgroup diversity within the host ecosystem, some of the virus groups within the low-diversity population appear to possess a high degree of intraspecific sequence heterogeneity such that there may be many diverse strains or moieties within each group. The temporal stability of certain viral sequences in an environment, and the influence physiochemical factors have on determining the types of viruses and viral genes present in a particular environment, are intriguing yet highly complex questions worthy of further detailed study.

New metagenomic surveys of low-diversity extreme environments like BSL may also help provide a framework for generating hypotheses that address the role viruses play in generating novel genes and their influence on the evolution and ecological 
structure of host communities. Current BLAST-based assessment of sequence reads in a viral metagenome (possibly quite substantially) overestimates the true extent of diversity within the virosphere, because some of this sequence "dark matter", while uncharacterized, is not in fact unique. Upon closer inspection, many of the unknown genes in the BSL virome appear to be co-localized with identifiable viral genes in the same contig (Figure 1.6) While such genes may certainly be novel, unknown genes or sequences should not be summarily misconstrued a priori as being indicative of novel virus genera or species. Nevertheless, the dark matter of virus sequence-space remains the predominant component of BSL and other natural virus assemblages (Figure 1.7), and this observation upholds the conclusion that viruses represent vast reservoirs of genetic novelty in the biosphere.

The purpose served by maintaining such a vast stockpile of novel viral genes in the environment, the origin of these unique viral sequences, and the mechanisms by which they are generated all remain central unresolved questions in the field of virus ecology [57, 66-69]. As more viral metagenomic surveys are conducted and made available in public databases, and as data analysis pipeline technology progresses, researchers in the field of virus ecology and genomics will be better poised to address these and other complex questions. 


\section{MATERIALS AND METHODS}

\section{Metagenomic DNA sample preparation:}

In late September 2009, approximately 20 liters of sediment slurry (roughly composed of 50\% solids) was collected from approximately one meter below the water surface at the north "Site-A" shoreline of Boiling Springs Lake (BSL) [2]. At this site, the water column temperatures are consistently $52+/-3^{\circ} \mathrm{C}$, with a pH of approximately 2.2 . The sediment was placed in a sterile carboy, then stored overnight at ambient air temperature. Sediment was transported for approximately 10 hours at ambient temperature before being placed in storage at $4^{\circ} \mathrm{C}$. Two liters of sterile PBS salt solution was then added to the $20 \mathrm{~L}$ of sediment slurry and mixed by shaking to detach viruses from the fine clay sediment particles. After allowing the BSL sediment-PBS salt solution to settle overnight at $4^{\circ} \mathrm{C}$, the aqueous layer (pore water) was siphoned off the top of the sediment. This procedure was repeated four more times, and the pore water-PBS was pooled in a separate carboy. The pore water-PBS was then pre-filtered with an inline 5 micron pore-size filter (Pall Profile II) prior to concentration by tangential flow filtration (using hollow-fiber TFF) with a $10 \mathrm{kDa}$ cutoff (Pall Microza). The native pore water-PBS was concentrated to approximately $70 \mathrm{~mL}$ prior to buffer exchange, which was carried out by the gradual addition of $500 \mathrm{~mL}$ of SM phage buffer $(100 \mathrm{mM} \mathrm{NaCl}, 10 \mathrm{mM}$ $\mathrm{MgCl}_{2}$ and $50 \mathrm{mM}$ Tris $\mathrm{pH}$ 7.0) while continuing the TFF concentration process, this time using a Pall Ultrasette TFF with a $100 \mathrm{kDa}$ cutoff until a final volume of $30 \mathrm{~mL}$ was reached. The virus concentrate was then split into two $15 \mathrm{~mL}$ aliquots. One $15 \mathrm{~mL}$ aliquot 
of BSL virus concentrate was passed through a Minisart $200 \mathrm{~nm}$ pore-size SFCA syringe filter (Sartorius-Stedim Biotech), prior to DNA extraction. The supernatant was then DNase-treated (final concentration of $2.5 \mathrm{U} / \mu \mathrm{L}$ for 30 minutes at $37^{\circ} \mathrm{C}$ ) to remove unencapsidated DNA. Virus-sized particles were disrupted by treatment in 10\% SDS and $2 \mathrm{mg} / \mathrm{mL}$ Proteinase $\mathrm{K}$ for 1 hour at $55^{\circ} \mathrm{C}$. A $1: 15$ volume of CTAB solution $(0.125 \mathrm{~g} / \mathrm{mL}$ hexadecyltrimethyl ammonium bromide in $2 \mathrm{M} \mathrm{NaCl}$ ) was added to remove dissolved polysaccharides and heated to $65^{\circ} \mathrm{C}$ for 10 minutes. Following CTAB treatment, phenol:chloroform:isoamyl alcohol (25:24:1) extraction of DNA was performed, and DNA was precipitated from the aqueous layer using 0.6 volumes of $-20^{\circ} \mathrm{C}$ isopropanol. The DNA extracted from virus-sized particles $(<200 \mathrm{~nm})$ was amplified with $\phi 29$ polymerase [70] (GenomiPhi v2, GE Healthcare Life Sciences). The amplified DNA was tested by PCR for microbial DNA contamination using the 515F/1492R universal 16S rRNA gene primer set [71] Reaction conditions as follows: 0.5 pmol of each primer was added to a PCR master mix. Sterile ddI $\mathrm{H}_{2} \mathrm{O}$ used in the master mix was used as a negative "no template" control. A ten-fold serial dilution of genomic DNA extracted from the Archaea, $S$ islandicus was used between $10 \mathrm{ng}$ to $1 \mathrm{pg} /$ reaction as a positive control. A crude lysate of $E$. coli was also used as a positive control for amplification of Bacteria and in the presence of contaminants that may inhibit the reaction. A ten-fold serial dilution of the BSLM $\phi 29$ amplified DNA from $10 \mathrm{ng}$ to $1 \mathrm{pg} /$ reaction was prepared to test for the presence of microbial DNA in the BSLM sample. All control and unknown sample tubes were run simultaneously in the thermocycler for; 10 minutes at $95^{\circ} \mathrm{C}$ initial denaturation, followed by $30 \mathrm{X}\left[94^{\circ} \mathrm{C}\right.$ for $30 \mathrm{sec} ., 55^{\circ} \mathrm{C}$ for $30 \mathrm{sec}, 72^{\circ} \mathrm{C}$ for 1 
min.], with a final extension for $5 \mathrm{~min}$. at $72^{\circ} \mathrm{C}$. One microgram of $\phi 29$ amplified BSLM DNA was ran on the gel directly, next to the PCR reactions, as a control to show that DNA was present in the BSLM reactions. Upon confirmation that the BSLM virus DNA sample contained very little microbial DNA, the sample was sequenced at the Broad Institute using Roche 454 FLX Titanium reagents on a half-plate as part of the Gordon and Betty Moore Foundation's Marine Microbiology Initiative [72]. The metagenomic data resulting from this sequencing run is referred to herein as "2009 BSLM-A1". After approximately two months in storage at $-20^{\circ} \mathrm{C}$, another portion of the DNA sample was amplified using $\phi 29$ polymerase, and sequenced as above to generate dataset "2009 BSLM-A2". BSLM-A1 (GAIR4WK04) and BSLM-A2 (GNX3RNF02) represent datasets from two independent $\phi 29$ amplifications of the same original BSL DNA sample. The BSL viral metagenomic sequences from both BSLM-A1 and A2 were combined into a single FASTA text file in silico, resulting in a combined sequence dataset herein referred to as "2009 BSLM".

Metagenomic sequence processing, contig assembly and ORF prediction:

The raw "2009 BSLM" metagenomic data was quality-filtered and de-duplicated using GenomeQuest read processing workflow (www.genomequest.com), resulting in the retention of $92 \%$ of the original reads. Quality-filtered reads were assembled using Newbler v2.0 (Roche 454 sequencing) requiring a minimum sequence match identity of $98 \%$ spanning an overlap length of 20 nucleotides. 
A dataset comprising 696 contigs and 229 singleton reads longer than 500 bases was manually extracted and uploaded to MetaVir ${ }^{2}$-Beta [26], which predicts both whole and partial ORFs from fragmentary metagenomic sequence data. Sequences shorter than 500 nucleotides were manually removed from the dataset.

Taxonomic composition of the BSL virus assemblage based on sequence data:

The PHACCS tool [27] was used to estimate the number and distribution of genotypes within the sample. Taxonomic composition of the BSL virus community was inferred by sequence-similarity of contig-derived ORFs to known viral genes. Automated taxonomic assessment was conducted using MetaVir ${ }^{2}$-Beta [26] which implements a tBLASTn-based query [73] of ORFs against the NCBI viral genomes database [74]. An E-value threshold of $10^{-3}$ was used to establish the presence of BSL ORFs matching known viruses. The resulting data was downloaded from MetaVir ${ }^{2}$-Beta and manually reconfigured in Excel to generate a stacked bar graph illustrating the most abundant, identifiable viral sequences in the metagenomic dataset arranged according to host domain, genomic configuration and putative virus family of origin.

Sequence heterogeneity among viral genes in BSL:

MetaVir [26] was used to map BSL metavirome sequence reads to known viral reference genomes using the "recruitment plot" tool. Fourteen pertinent reference genomes matching reads from the BSL metavirome were selected for analysis in order to illustrate the relatedness between the viruses found in BSL and their counterparts in the 
NCBI virus database [74]. The data generated by MetaVir includes the position and span of mapped reads within the context of the reference genome, as well as tBLASTx [73] Evalues. These data were used to generate a box-and-whisker histogram plot of various viral genes, indicating the coverage or span of the region of the reference genome that BSL sequence reads were mapped to, and the amino acid sequence identity shared among the query and reference sequences.

\section{Comparative rarefaction analysis of BSL sequences and other viral metagenomes:}

To view contrasting patterns of metagenomic sequence heterogeneity spanning multiple ecotypes, rarefaction curves from publically available viral metagenomes in MetaVir were compared to those from BSL. Individual metagenomic sequence reads were clustered by rarefaction by applying $98 \%, 90 \%$ and $75 \%$ sequence identity stringency cut-off values. Rarefaction endpoints, or "read-per-cluster" ratios are calculated by dividing the total number of raw reads in the metagenome by the number of clusters formed by rarefaction at a given stringency. The read-per-cluster ratios for each selected metagenome, calculated at each stringency level, were plotted in a 100\% stacked bar graph to illustrate intraspecific sequence heterogeneity within, and differences in sequence richness among the various ecotypes.

Long-term detection of viral genes in BSL sediment samples by PCR:

DNA from BSL sediment samples collected in 2010, 2011 and 2012 was isolated and tested for the presence of virus sequences derived from the 2009 BSLM dataset by 
PCR. The 2009 BSLM DNA used for metagenome sequencing was used as a positive control. The 2010 and 2012 samples were prepared in a similar manner to that of the 2009 BSLM sample, except that virus particles were detached from BSL sediment by increasing the $\mathrm{pH}$ of the sample as follows: Unbound virus particles were first harvested from 1 liter of BSL sediment-pore water slurry ( $c a .1: 1)$ obtained from BSL Site-A, from approximately one meter below the water surface. The liter of sediment slurry was divided among sixteen $50 \mathrm{~mL}$ conical centrifuge tubes and all tubes were centrifuged for 10 minutes in a Jouan CR412 swinging bucket rotor centrifuge at 3,000 r.p.m.. Virus particles still adhered to the compacted, centrifuged sediment were subsequently detached by resuspending the sediment in a pH 9.0 SM phage buffer containing $100 \mathrm{mM}$ $\mathrm{MgCl}_{2}(100 \mathrm{mM} \mathrm{NaCl}, 100 \mathrm{mM} \mathrm{MgCl} 2$ and $50 \mathrm{mM}$ Tris, $\mathrm{pH}$ 9.0). Resuspension of the sediment by stirring and vigorous shaking of the conical $50 \mathrm{~mL}$ tubes by hand was followed by another round of centrifugation as described above. The SM buffer supernatant was concentrated by TFF separately from the native pore water to avoid precipitation of dissolved iron compounds that occurs if the $\mathrm{pH}$ of the acidic native pore water is raised. Following TFF concentration, both adherent and non-adherent virus particle fractions were filtered through a Minisart $200 \mathrm{~nm}$ pore-size SFCA syringe filter (Sartorius-Stedim Biotech). Concentrated non-adherent viruses were concentrated further using a $15 \mathrm{~mL}$ disposable ultrafiltration device (Vivaspin® PES, $100 \mathrm{kDa}$ cutoff, Sartorius-Stedim Biotech). SM phage buffer at a pH of 7.0 was then added to the concentrate remaining in the ultrafiltration column, which was then re-centrifuged to exchange the buffer in both virus suspensions. Both adherent and non-adherent SM 
buffer-exchanged suspensions were combined prior to DNA extraction using the method previously described for the preparation of the 2009 BSLM-A1 and A2 samples.

Samples collected in 2011, however, were obtained from both site-A and "siteD". BSL site-D is in the vicinity of several exposed and shallow submerged fumaroles and has a temperature of approximately $93^{\circ} \mathrm{C}$ and a $\mathrm{pH}$ of approximately 2.0 [2]. The 2011 sediment samples were prepared using a filter-based approach, such that the 0.2 micron pre-filtered native pore water and SM buffer supernatants were passed through a 25 nanometer pore-size mixed cellulose ester filter (Millipore VSWP), using a frit and funnel apparatus to extract virus particles instead of using TFF. The captured material was then rinsed off the filter using a wash solution $(400 \mathrm{mM} \mathrm{NaCl}, 750 \mathrm{mM}$ Sucrose, 20 mM EDTA, $50 \mathrm{mM}$ Tris-BASE, 1\% v/v Triton X-100 and 1\% w/v SDS adjusted to $\mathrm{pH}$ 7). DNA was then extracted from the wash solution using the same protocol as described above for the 2009 BSLM, 2010 and 2012 samples.

DNA samples prepared from site-A are; "2010 BSL vDNA-A", "2011 BSL vDNA-A" and "2012 BSL vDNA-A". The only sample obtained from site-D is "2011 BSL vDNA-D”. All DNA samples were amplified with $\phi 29$ polymerase prior to use in PCR. A series of all samples, including the positive control and water blanks, were aliquoted and stored at $-20^{\circ} \mathrm{C}$ prior to PCR testing for the presence of putative viral sequences. A master mix containing a specific primer set was added to a freshly thawed DNA sample series, and the reaction tubes were ran simultaneously in the thermocycler using the same cycling parameters. 
PCR primers were designed manually based on 2009 BSLM sequence data. PCR primer sequences and amplicon lengths of each of the sets used in this analysis are shown in Table 1.2.

Defining ORF-types in the BSL contig dataset:

Translated ORF sequences that did not result in a sequence match to the NCBI virus database by tBLASTn were designated by MetaVir'-Beta as "'unaffiliated". The unaffiliated BSL ORF sequences were then uploaded to the CAMERA-2.0 portal (http://camera.calit2.net) [28] and queried against the CAMERA virus database in order to identify additional putative viral ORFs. The unaffiliated ORFs were also searched against the CAMERA reference sequence database to identify possible cellular or "hostassociated" genes. The remaining unknown ORF were considered putative "dark matter" ORFs [29] or "ORFans" [30], indicating that the sequence has no detectable similarity to other coding sequences in any database. Putative dark matter ORFans were searched against 12 metagenomes (summarized in Table 1.1) in the CAMERA database using tBLASTn to identify common, conserved hypothetical proteins designated herein as "gray matter" ORFans.

Contextual assessment of ORF-types by contig association

To visualize the distribution and co-occurrence of ORF types within specific putative virus genomes and genome fragments, BSL contigs were first sorted by size. For 
the largest 7 contigs, the number of ORFs of the following type; virus, host-associated, gray matter, and dark matter, were plotted in a $100 \%$ stacked bar graph.

Rarefaction or "protein clustering" of the ORF amino acid sequences was done to remove redundancy and to estimate the number of protein families in BSL according to ORF-type. Category-specific ORF amino acid sequences were uploaded separately into MetaVir, and rarefaction was performed at low stringency (75\% identity) on each of the individual ORF-type datasets. The resulting number of protein clusters formed per ORFtype was plotted in relation to the total number of predicted ORFs per category in a bar graph.

BSL gene content cross-comparison:

The set of 229 viral, host-associated and gray matter BSL ORFan nucleotide sequences producing a significant tBLASTn hit in at least one of the 12 selected CAMERA metagenomes were annotated according to the BSL ORF sequence identifier, the location in which a homolog was found, and the $-\mathrm{LOG}_{10}$ E-values of the tBLASTn hit. These data were plotted in an X-Y bubble plot to illustrate the distribution of and general sequence similarity between BSL ORFs and their cognate sequences in other biomes.

The "Data Overlapping and Area-Proportional Venn Diagram" tool at BioInfoRx (http://apps.bioinforx.com) was used to specifically analyze the BSL gray matter ORFans. This allowed for a more detailed assessment of the conserved proteins of unknown function with respect to the physiochemical environments in which they are 
found. These gray matter ORFan Venn data were used to generate a Cytoscape [75] plot (www.cytoscape.org/) showing the $-\mathrm{LOG}_{10} \mathrm{E}$-value of the BSL sequence match, and the other environments in which a homolog was found. The BSL ORFan sequence identifier was used as the "source interaction" data, and the metagenome in which a similar sequence was found was used as the "target interaction" data. The $-\mathrm{LOG}_{10} \mathrm{E}$-value of each hit was used as an "edge attribute" so that the thickness of the connecting line could be modified to depict the quality of each match. Node attributes were modified manually to indicate whether the subject database was a microbial or viral metagenome. 


\section{CHAPTER 1 FIGURES:}

Figure 1.1 Predicted taxonomic composition of the BSL virus assemblage

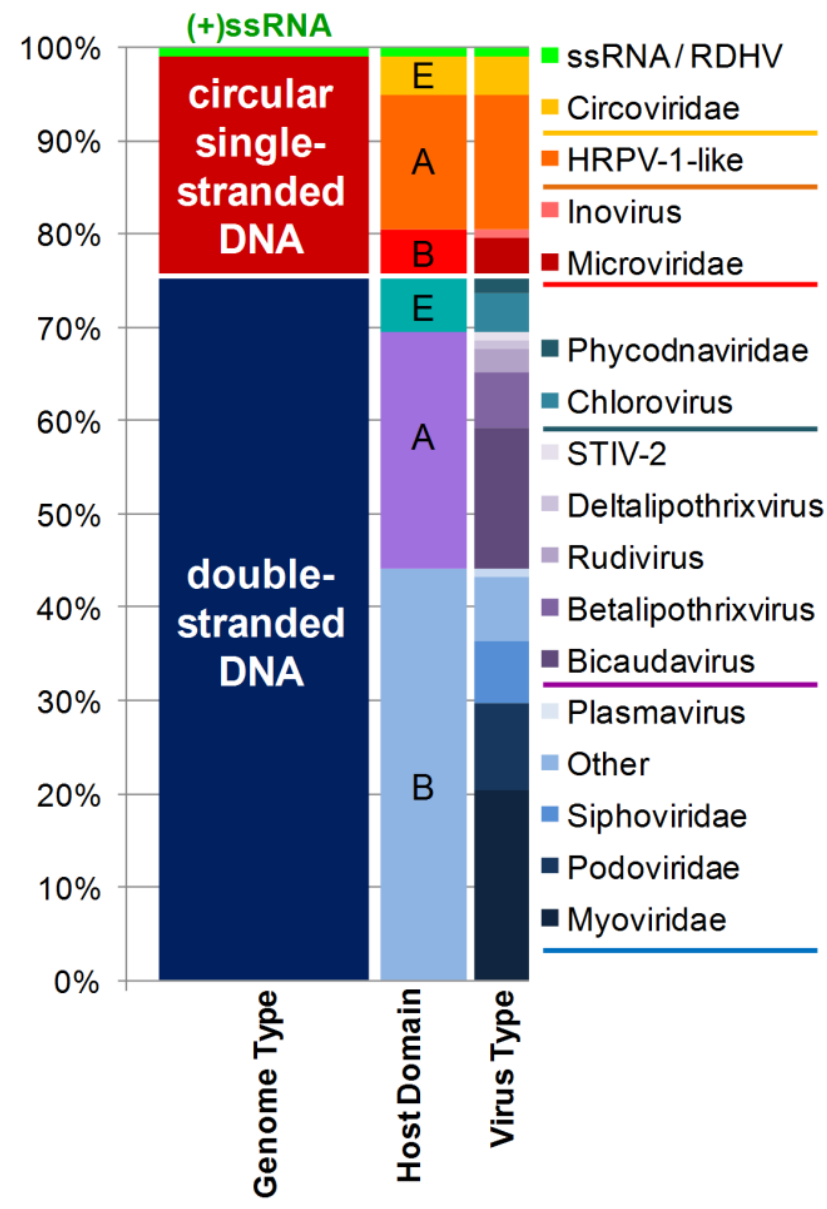

Taxonomic composition of the 2009 BSLM virus community was inferred by tBLASTn sequence-similarity of contig-derived ORFs to known viral genes. Automated taxonomic assessment was conducted using MetaVir ${ }^{2}$-Beta. The principal virus genome types identified based on similarity to known virus genes are: dsDNA viruses (navy blue), circular ssDNA viruses (red) and ssRNA viruses (green). Each genome type is further subdivided according to "host domain", indicating whether the virus matched infects organisms from the domain Bacteria (B), Archaea (A) or Eukarya (E). Host domains are subdivided and color coded by "virus type". Horizontal colored lines separate the virus types and are color-coded according to the host domain they are known to infect. 
Figure 1.2 Recruitment plot data of select virus-affiliated sequences

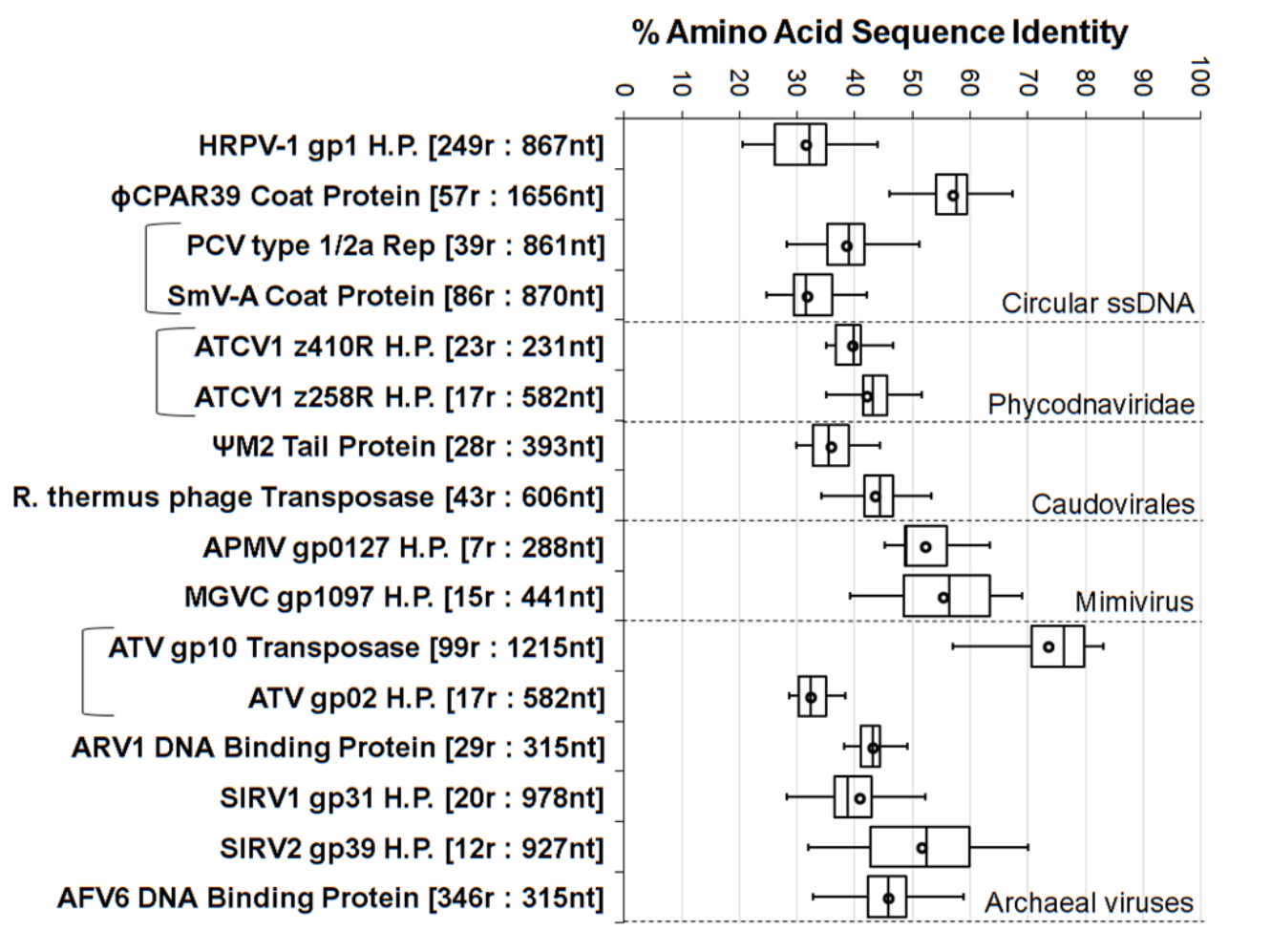

Box-and-whisker plots represent the overall amino acid sequence identity, based on local alignment, between translated BSL sequence reads and cognate reference genes from the MetaVir virus sequence database. The number of BSL reads mapping to a reference sequence is shown in brackets with the suffix (r) and the second number labeled with the suffix (nt) indicates the number of bases spanned by the recruited BSL reads along the reference sequence. Brackets encompassing two sequence names indicate that the genes are from the same virus. (e.g., PCV Rep and SmV-A CP are both associated with RDHV - see CHAPTER 3). Hypothetical Protein reference sequences are indicated by the abbreviation "H.P.". Low whisker = minimum observation below upper fence $[1.5 \mathrm{x}$ Inter Quartile Range (IQR)], low box $=25^{\text {th }}$ percentile of IQR (middle half of data), box divider $=$ median, upper box $=75^{\text {th }}$ percentile of IQR, upper whisker $=$ max observation below upper fence [1.5xIQR], 'o'= mean. (HRPV-1: Halorubrum pleomorphic virus-1, phiCPAR39: Microviridae, PCV: Porcine circovirus, SmV-A: Sclerophthora macrospora virus-A, ATCV1: Acanthocystis turfacea chlorella virus-1, psiM2: Methanobacterium phage, APMV: Acanthamoeba polyphaga mimivirus, MGVC: Megavirus chilensis, ATV: Acidianus two-tailed virus, ARV1: Acidianus rod-shaped virus-1, SIRV1 and 2: Sulfolobus islandicus rod-shaped virus-1 and-2, AFV6: Acidianus filamentous virus-6.) 
Figure 1.3 Comparative rarefaction analysis of multiple metaviromes

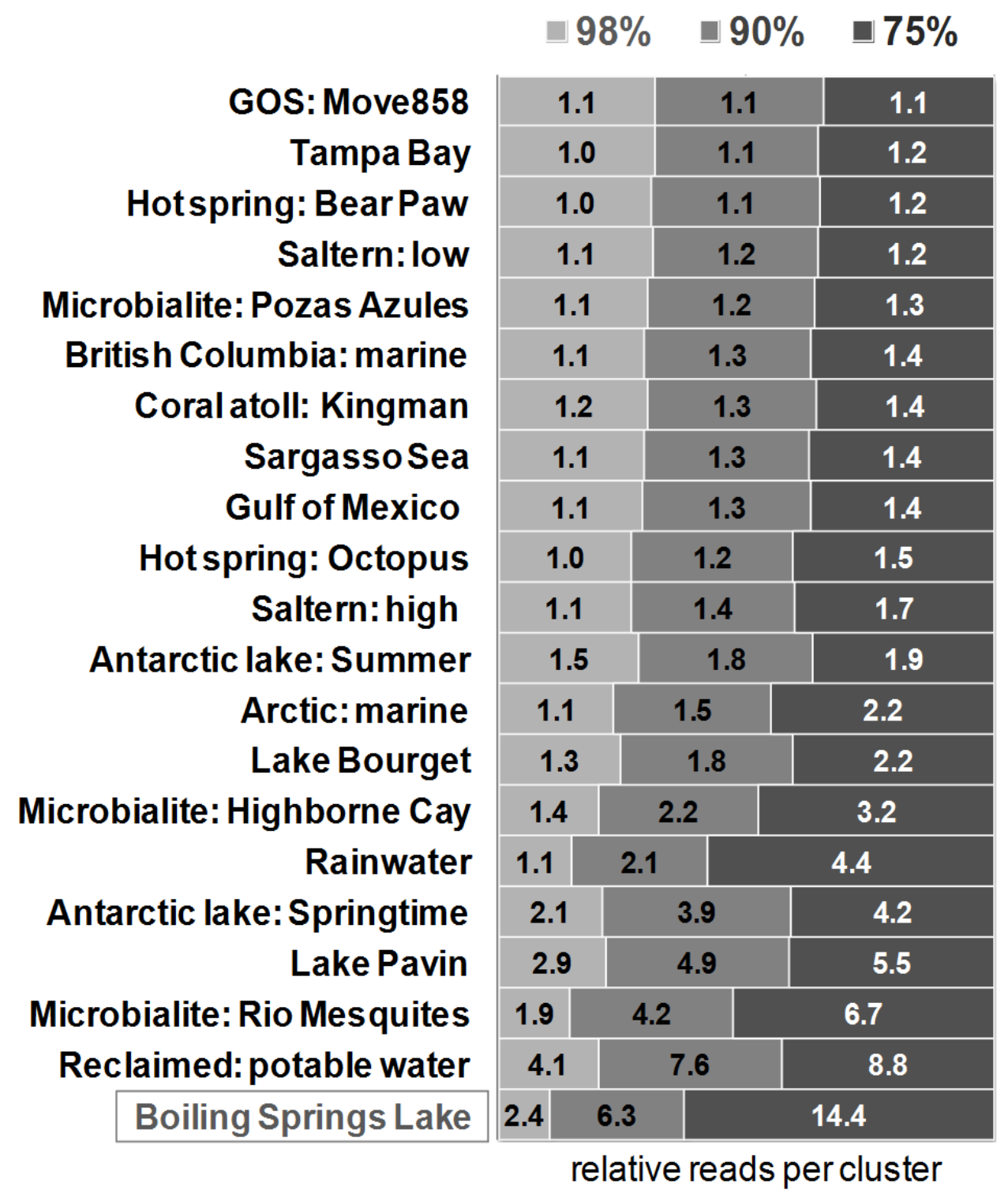

Metavirome sequence diversity as indicated by rarefaction. Read-per-cluster ratios are calculated manually based on MetaVir rarefaction data by dividing the total number of metagenomic sequence reads by the number of homologous clusters formed by rarefaction at three different nucleotide sequence identity stringencies; $98 \%, 90 \%$ and $75 \%$. Ratios are proportional to the level of intraspecific heterogeneity, whereas a near equal ratio at all stringencies indicates that most sequence reads are unique. Ratios are plotted in a stacked bar graph format indicating the relative differences in the read-percluster ratios formed at each stringency setting. Numbers within each bar indicate the read-per-cluster ratio at each rarefaction stringency. 
Figure 1.4 Long-term PCR testing for virus-like sequences in BSL

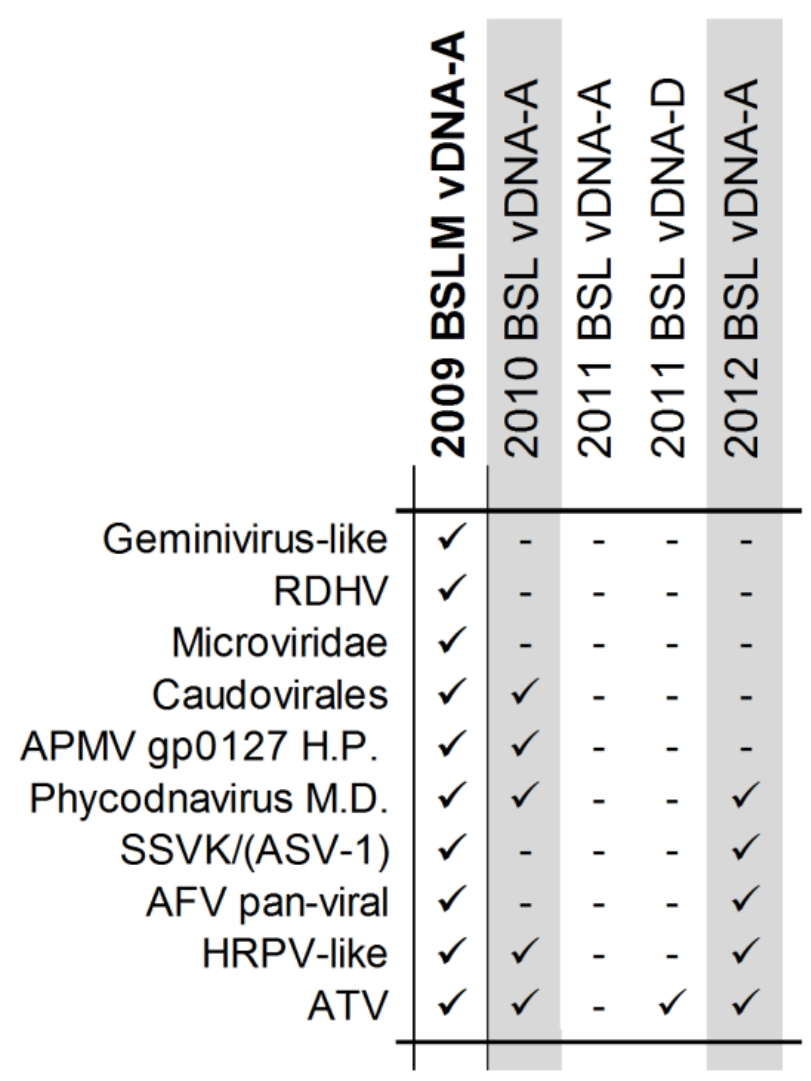

PCR primer sets were designed to amplify ten different virus-affiliated gene sequences from the BSL metavirome. Each PCR reaction contained $10 \mathrm{ng}$ of DNA derived from virus-sized particles (vDNA) collected in 2010, 2011 and 2012 from BSL sites " $A$ " and "D". The DNA used for metagenomic sequencing (2009 BSLM) served as the positive control template for all samples. A $(\checkmark)$ indicates that a PCR product of the correct size was generated. Negative results, characterized by a lack of amplification when the positive control and other samples produced an appropriate amplicon, are indicated by a (-) symbol. Primer sequences and amplicon lengths are provided in Table $\mathbf{1 . 2}$ 
Figure 1.5 BSL gene homologs found in other metagenomes

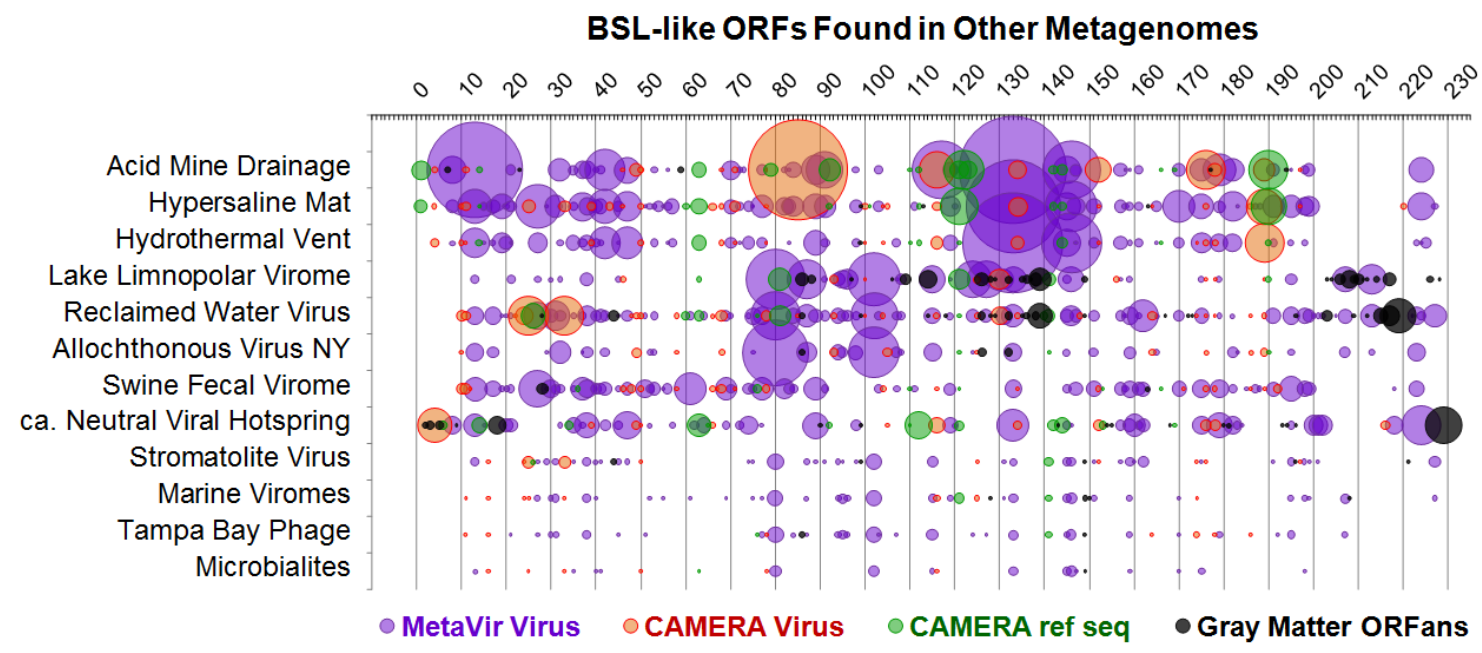

A total of 229 BSLM dataset ORFs with homologs in other metagenomes were queried against other non-annotated environmental virus metagenomes using tBLASTn. Sequences yielding E-values less than $1 \times 10^{-3}$ are plotted in this X-Y bubble plot. Of the 229 BSL ORFs screened, 141 are viral, 27 are "host-associated", and 61 are "gray matter" ORFans. A bubble denotes whether a similar sequence is present in another metavirome. The Y-axis identifies the metavirome dataset in which a homolog of the BSL ORF is found. The diameter of the bubble is proportional to the $-\mathrm{LOG}_{10} \mathrm{tBLASTn}$ E-value of the match between the BSL ORF and the reference sequence, such that larger bubbles indicate a more significant match. 


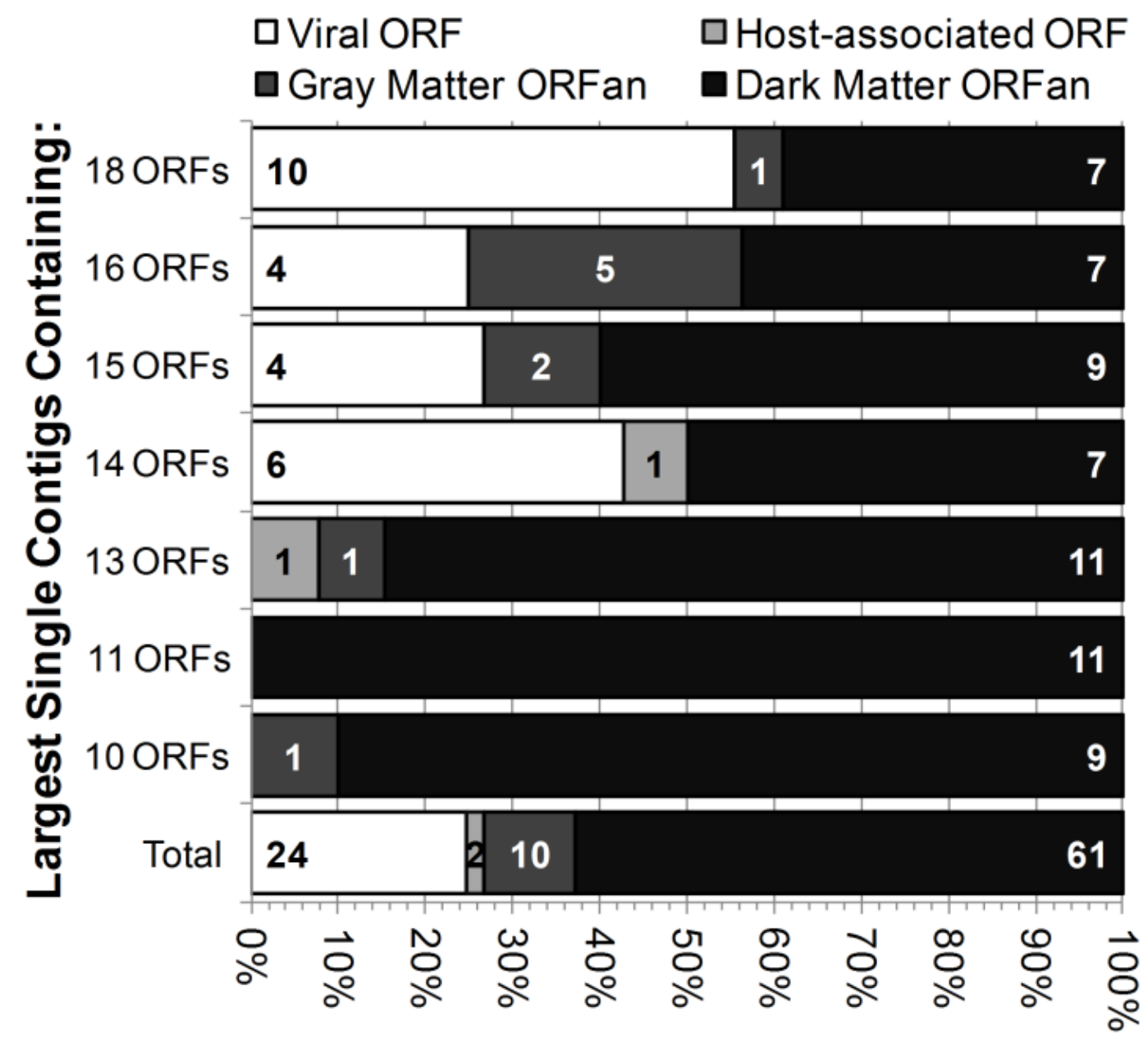

ORFs predicted within the 7 largest BSL metavirome contigs are labeled according to ORF classification to illustrate the co-localization of affiliated genes and ORFans on the same contig. White ORFs are similar to sequences in known viral genomes, "host associated" ORFs are similar to ORFs found in the CAMERA reference sequence database, "gray matter" ORFans are similar to ORFs found in other metagenomes, and "dark matter" ORFans have no detectable homolog in any sequence database. The X-axis of the $100 \%$ stacked-bar graph indicates the percentage of each ORF type within a given contig. Numbers within the bars indicate how many ORFs of a given type are found in a particular contig. The bottom bar is a summation of the contigs shown above, and the relative percentage of ORF types contained within all 7 contigs. 
Figure 1.7 Estimating the number of protein families in the BSL virus assemblage

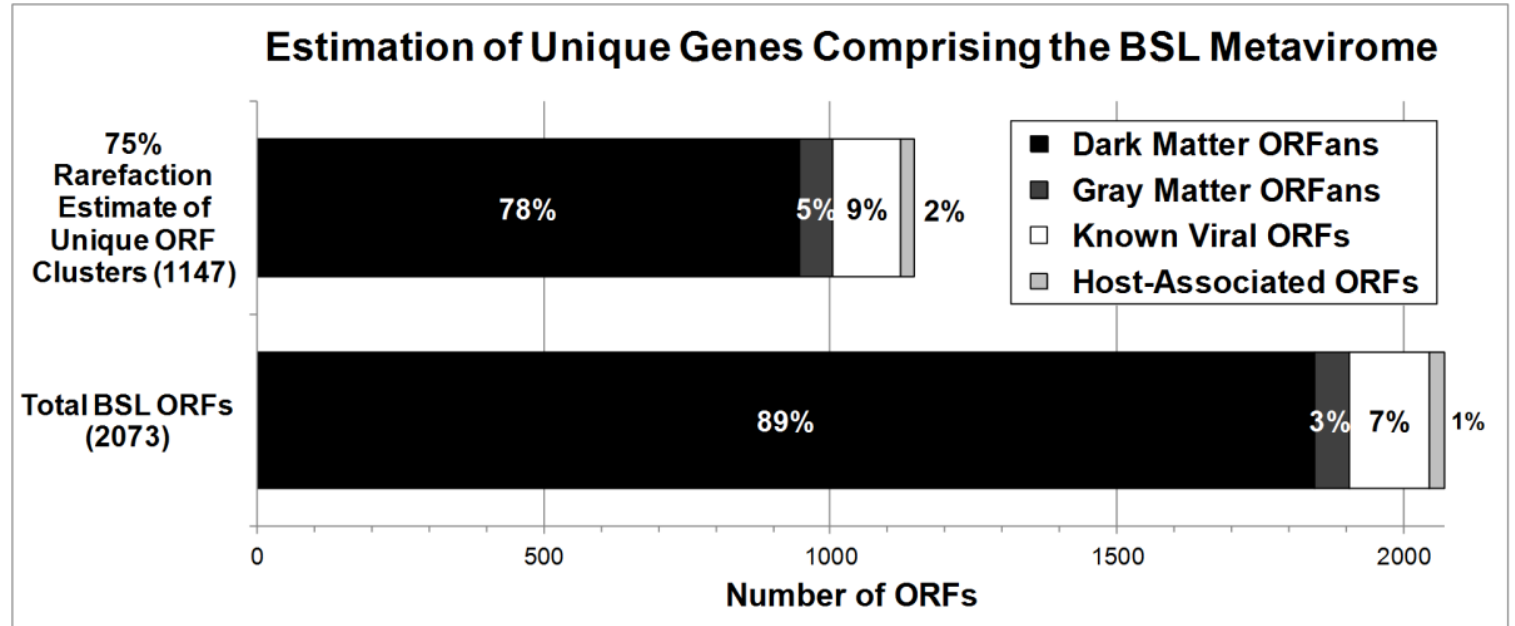

All ORFs predicted from the BSLM contigs and singletons longer than 500 bases were clustered at $75 \%$ amino acid sequence identity to estimate the number of protein families in the virus assemblage. Virus- and host-associated ORFs, as well as the "gray" and "dark matter" ORFan categories are clustered separately. The resulting number of protein clusters formed (1199) and percentages of each ORF category are compared to the total number of unclustered ORFs in all categories (2073). 
Figure 1.8 BSL "gray matter" ORFans sorted according to environment type

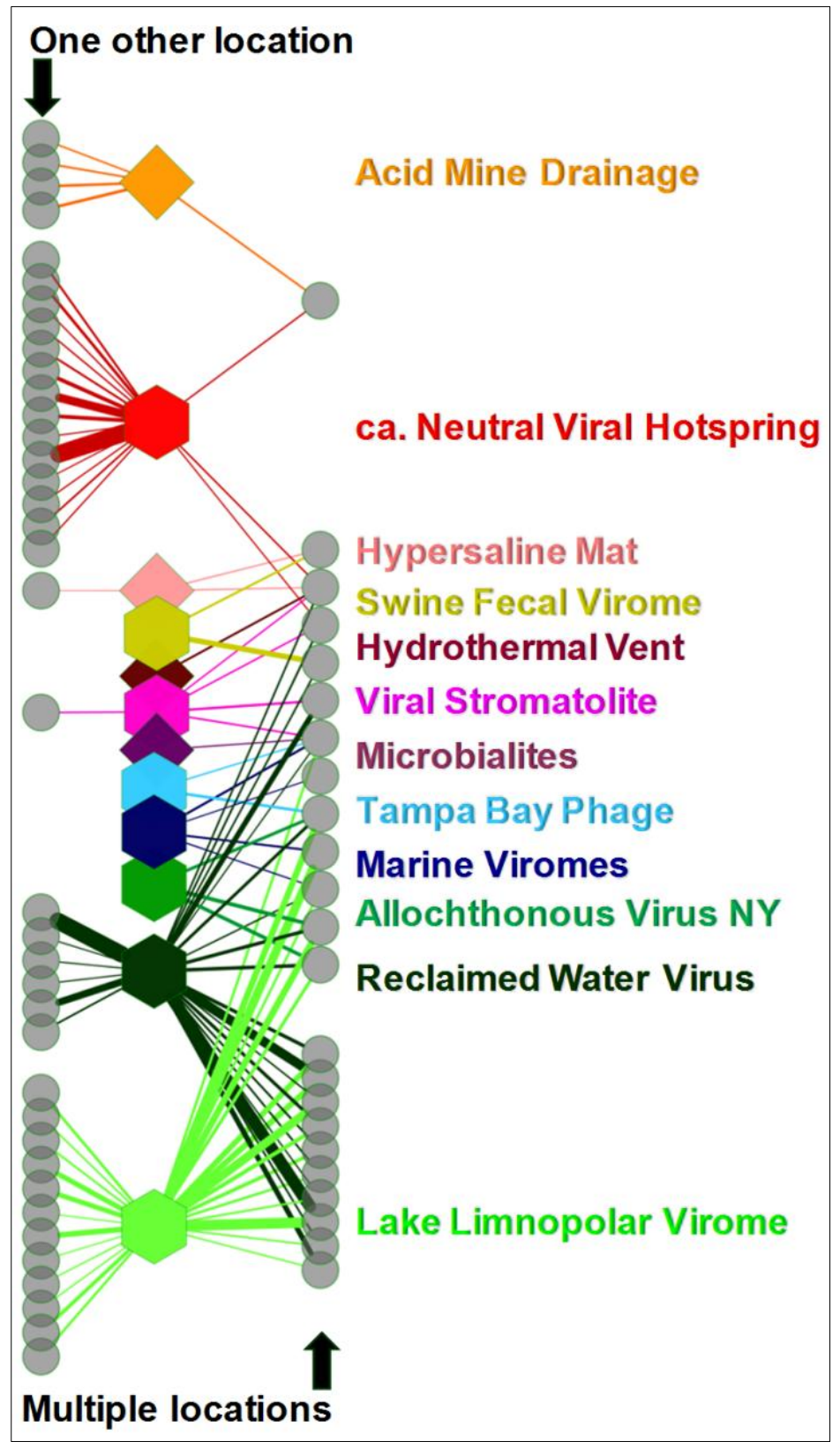

A Cytoscape network illustration of Venn analysis data indicates the location of 61 nonredundant BSL ORF "gray matter" homologs found in CAMERA database metagenomes. Line thickness is proportional to the tBLASTn - $\mathrm{LOG}_{10}$ E-value of the reference sequence match. Hexagons indicate a viral metagenome, diamonds indicate a cellular metagenome and gray circles indicate individual BSL "gray matter" ORFans; 38 of which are found in only one other location and 23 are found in multiple locations. 


\section{CHAPTER 1 TABLES:}

Table 1.1 Reference Metagenomes in the CAMERA Database Utilized in the BSL Comparative Analysis

\section{CAMERA Metagenomic Datasets}

LakeLimnopolarVirome: All Metagenomic 454 Reads (N)

TampaBayPhage: All Metagenomic 454 Reads (N)

HydrothermaIVent: All Metagenomic Shotgun Reads $(\mathrm{N})$

ReclaimedWaterViruses: All Metagenomic 454 Reads (N)

ViralStromatolite: All Metagenomic 454 Reads (N)

ViralSpring: All Metagenomic Sequence Reads (N)

Yellowstone: All Metagenomic Shotgun Reads (N)

AcidMine: Assembled Sequences (N)

Microbialites: All Metagenomic 454 Reads (N)

HypersalineMat: All Metagenomic Shotgun Reads (N)

Allochthonous viruses in upstate NY freshwater resources: Assembled sequences $(N)$

Swine fecal viromes: All Metagenomic 454 Reads (N)

MarineViromes: All Metagenomic Sequence Reads (N) 
Table 1.2 BSL Virus Primer Information
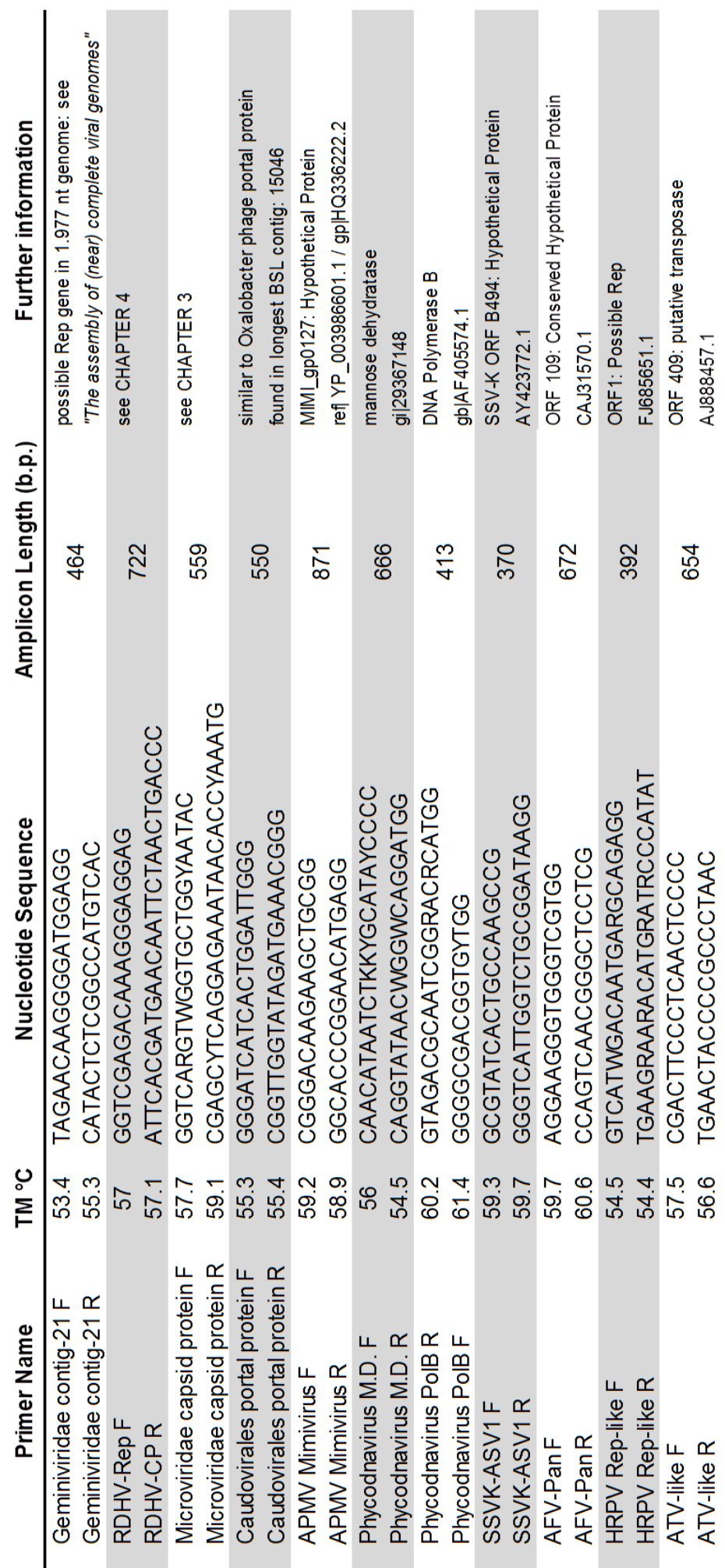


\section{CHAPTER 2:}

\section{Utilizing Boiling Springs Lake Viral Metagenomic Sequence Data to Model the}

Structural Dynamics of Gokushovirinae Capsid Protein Evolution.

\section{CHAPTER 2 ABSTRACT}

The Microviridae are a family of small, icosahedral viruses that are somewhat like the viral equivalent of "Darwin's finches" — this globally ubiquitous and highly sequence-diverse group of viruses may provide valuable insights into how viruses evolve and adapt to vastly different environments. Microviruses have small genomes and relatively simple, icosahedral virion structures, which are remarkably similar to each other structurally, despite extensive sequence diversity within the group. Adaptations generated by natural selection, as well as ancestral relationships within this diverse group of viruses, are principally revealed herein by way of structure-based protein sequence analyses utilizing metagenomic sequence data from Boiling Springs Lake (BSL) and other surveys. The discovery of two microvirus genomes in the BSL viral metagenome has expanded the range of possible habitats that these viruses are now known to inhabit. New sequence data from metagenomic surveys, encompassing diverse environments such as BSL, will be utilized herein in order to better ascertain how these viruses have evolved, and how they have adapted to different environments. The wealth of new sequence data from metagenomic surveys is utilized to dissect the fundamental physical determinants underlying virion assembly in this highly sequence-diverse group of viruses. 


\section{INTRODUCTION}

The Family Microviridae is subdivided into four subgroups: Microvirus, Gokushovirinae, Pichovirinae and Alpavirinae [45]. Microviruses infect Enterobacteria, but well-characterized members of the Gokushovirinae have so far only been shown to infect obligate intracellular parasites within the genera Chlamydia, Bdellovibrio and Spiroplasma [76], and are thus commonly referred to as "parasites of parasites". The Pichovirinae, primarily categorized as such based on differences in genomic organization relative to the other subgroups, may also be distinguished through protein sequence alignment-based comparisons [45]. All of the Alpavirinae identified thus far have been found as lysogenized prophages within the genomes of Bacteroidetes [77].

Two microvirus genomes have been completely reconstructed from the Boiling Springs Lake (BSL) metavirome sequence assembly (CHAPTER 1). These two circular genomes were also amplified from the original metagenomic DNA sample by inversePCR, and one has been completely re-sequenced to confirm the fidelity of the metagenomic contig assembly. The host organism(s) for the BSL strains have not yet been identified, however, it is possible that they infect mollicutes or chlamydia-like organisms. Freshwater amoeba are known to be natural hosts for Chlamydiales species in the environment, even though they are commonly known as intracellular parasites of human macrophages [78]. Thus the amoebal species found in BSL [3] might be hosts for chlamydia-like organisms and, in turn, for the BSL microviruses. However, no Chlamydiales have been yet found by 16S rRNA surveys of BSL [4]. The Microvirus 
subgroup of viruses has been shown to be quite flexible in its ability to infect a broad range of hosts due to its ability to recognize LPS as a receptor, thus it is possible that free-living Gram negative bacteria in BSL may eventually be shown to harbor these viruses [4].

Microviruses have circular DNA genomes, roughly 4-6 kb in length, that are packaged inside the virion as single-stranded DNA, and are comprised of at least five principal protein-encoding genes. Gokusho-, Picho- and Alpavirinae designations are given first, and Microvirus homologues are shown in parentheses. Vp1 (F) is the major capsid protein, $\mathrm{Vp} 2(\mathrm{H})$ is the DNA pilot protein, Vp3 (B) is the internal scaffolding protein, Vp4 (A) is the rolling circle replication initiation protein "Rep", and VP5 (J) is a DNA binding protein. The Vp1 major capsid protein (MCP) is the subject of this investigation.

The structure of the $\varphi$ X174 microvirus, including an individual structure of its MCP, was experimentally determined by X-ray crystallography over two decades ago [79] The structure of the related Spiroplasma phage-4 (SpV-4) gokushovirus, which infects the mollicute Spiroplasma melliferum, was determined by cryo-electron microscopy (cryo-EM) to $27 \AA$ resolution, using the microvirus $\varphi$ X174 crystallographic structure as a template [80]. All virions within the family Microviridae have $\mathrm{T}=1$ symmetrical icosahedral virions, approximately $50 \mathrm{~nm}$ in diameter. The $\mathrm{SpV}-4$ virion is composed entirely of 60 identical major capsid protein subunits. The core of the major capsid protein structures of $\varphi \mathrm{X} 174$ and SpV-4 consist of an anti-parallel 8-stranded $\beta$ barrel in a jelly-roll configuration (B-C, D-E, F-G, and H-I strands), which lies within the 
roughly spherical surface of the virion. There are seven insertions between the strands, each of variable length, that embellish the $\beta$-barrel core structure. However, in the SpV-4 gokushovirus the insertion number 5, positioned between the $\mathrm{E}$ and $\mathrm{F}$ strands of the beta barrel, is by far the longest of the 7 insertions and forms a semi-globular tri-fold loop structure that extends roughly perpendicular from the main body of the capsid protein by a stalk-like $\alpha$-helix. Insertion 5 is situated directly in the middle of the amino acid sequence in all known Gokusho-, Picho- and Alpavirinae MCPs [45]. In SpV-4, three of these tri-fold loop extensions from separate MCPs come together in the complete virion to form a mushroom-shaped spike, approximately $5 \mathrm{~nm}$ in length, that protrudes from the 3 -fold symmetry axis vertices of the virion. The end of each spike trimer contains a small hydrophobic binding pocket, which is thought to play a role in host recognition [81]. Moreover, a much greater level of sequence heterogeneity within insertion-5 is always observed relative to the rest of the capsid protein, indicating strong positive selection in this specific region, which is thought to allow the virus to adapt to rapidly changing host receptor epitopes [80, 81]. The Microvirus subgroup is primarily distinguished from the Gokusho-, Picho- and Alpavirinae sub-groups by its lack of the insertion-5 derived spike structure. Viruses within the Microvirus sub-group, such as $\varphi \mathrm{X} 174$, instead encode a separate protein "G", which forms pentameric spike structures that decorate the 5-fold vertices of the virion [81].

Each roughly triangular MCP interacts with others at all three of its edges, leaving one face exposed to the environment, and the other facing the interior of the virion where the viral DNA is packaged. Protein-protein interaction interfaces assemble at 2-fold, 3- 
fold and 5-fold axes of symmetry to produce virions. Given that there is extensive sequence divergence within this group of viruses, it is these interfaces that are expected to be most strongly affected by purifying selection, in contrast to the tri-fold loop region, where positive selection is thought to be favored [80,81]. Maintenance of function, substrate binding, mechanical motion, core stability and quaternary interactions are generally maintained through the conservation of key amino acids [82]. Purifying selection that is responsible for maintaining functionality, quaternary structural interactions and internal stability, however, must be balanced with positive selection that drives adaptation and evolution [83]. While it is, in fact, possible to study the structural dynamics of microvirus capsid protein evolution in response to very specific environmental changes, such as an increase in temperature for example, these systems must be studied in vitro [84]. Structurally congruent yet sequence-diverse capsid proteins of the Microviridae family make ideal models for studying the structural dynamics of protein evolution because they must form several stable quaternary interactions and maintain mechanical function, while adapting to different physiochemical environments, or to overcome host resistance to infection [85].

As large numbers of new metagenomic sequences become available, and as more viral protein structures are determined by cryo-EM and crystallographic modeling methods, these efforts may be synergistically combined to create a much richer data landscape that will greatly enhance the study of viral protein structural dynamics and evolution. Due to the highly divergent DNA and amino acid sequences observed throughout the Microviridae subgroups, structure-based analyses are likely to be the most 
reliable means of determining the effects of natural selection within highly diverse protein families $[86,87]$. Two BSL gokushovirus isolates are compared by way of structure-based analysis to other well-characterized and environmental capsid sequences in order to: 1) determine whether patterns of amino acid sequence conservation are associated with capsid protein-protein interaction interfaces required for proper assembly of the virion, and 2) investigate other potential phenotypes of the BSL virus capsid proteins which may be specific adaptations to a hot, acidic environment.

\section{RESULTS AND DISCUSSION}

\section{Phylogenetic assessment of the BSL microvirus major capsid proteins}

Major capsid protein (MCP) genetic and translated sequences of the Microviridae family were compared using BLAST (Table 2.1). Weak tBLASTn E-values demonstrate that nucleotide sequences are poor estimators of relatedness between microvirus capsid protein genes, and that despite rather low percent identities, BLASTp comparison of amino acid sequences is in many instances the only reliable means of correctly identifying a viral protein $[86,88,89]$. Highly divergent DNA sequences, such as the microvirus capsid genes used in this analysis, are not suitable for evolutionary assessment because it is difficult to generate an adequate codon-based sequence alignment. Without proper codon alignment, it is not possible to identify or to evaluate the rate of synonymous and non-synonymous substitutions. Therefore, a Neighbor-Joining phylogenetic tree was constructed from an alignment of 27 capsid protein amino acid 
sequences, encompassing the Gokushovirinae, Pichovirinae and Alpavirinae microvirus subgroups, in order to resolve clades of related capsid proteins. The tree was rooted with the Enterobacteria phage $\alpha 3$ and $\varphi$ X174 microvirus capsid protein genes (Figure 2.1).

Phylogenetic analysis of capsid protein amino acid sequences indicates that the BSL isolates are grouped within a clade of gokushovirus that have been identified in metagenomic datasets obtained from both freshwater and marine environments (one gokushovirus from Lake Bourget in France $[44,45]$ and four from the Global Ocean Survey "GOS") [90]. The environmental clade of gokushoviruses that contains the BSL isolates appears to constitute a lineage that is distinct from clinical and laboratory isolates such as the Chlamydia phages, as well as the SARss-type marine viruses [51]. Since the GOS and SARss strains both originate from marine (and possibly estuarine) environments, it is likely that their placement in different clades is due to divergent coevolutionary associations with different host organisms. The BSL MCP sequences are more closely related to one another than they are to any of the other environmental isolates, despite sharing only $65 \%$ amino acid sequence identity between them. This not only illustrates the extensive range of sequence diversity that exists within this globally ubiquitous group of virus proteins, but it also underscores the ostensibly rapid rate of mutation and adaptation that these viruses are capable of sustaining (see also CHAPTER 1, Figure 1.2) [91, 92]. 
The structure of the major capsid protein and its assembly into virions

The SpV-4 gokushovirus (1KVP) [80] and the microvirus $\varphi$ X174 (2BPA) [79] MCP solved structures, and presumably the Picho- and Alpavirinae, have major capsid proteins that are arranged in a jelly-roll configuration, resulting in an 8-stranded $\beta$-barrel structure. Elaborate insertions, primarily located between the E and F strands, embellish the $\beta$-barrel and form the majority of the capsid protein structure (Figure 2.2). The trifold loop projection, a predominant distinguishing feature of the gokushovirus MCP, is not present in the Microvirus subgroup. The edges of the wedge-shaped protein interact and bind together during assembly of the virion at the 2-, 3-, and 5-fold axes of symmetry (Figure 2.3).

The 2-fold axis interaction involves the binding of two capsid protein monomers that are oriented at roughly 180 degrees to one another. A slightly S-shaped surface extending along the right edge of the protein (as viewed in Figure 2.3) interacts with its partner to form a dimer.

The 3-fold axis interaction forms the mushroom-shaped protrusion composed of three "stalks" that wrap around one another in a counter-clockwise orientation, and support a "cap" structure composed of three tri-fold loops. The interacting loops in the $\mathrm{SpV}-4$ model come together to form a hydrophobic pocket that is thought to play a role in host recognition and entry [80]. The amino acid sequences forming the tri-fold loop regions are highly variable among all known isolates of the Gokushovirinae. It is believed, therefore, that this protrusion is somewhat like an antibody Ig domain, in that residues exposed at the top of the protrusion allow it to recognize and bind host receptor 
epitopes [80]. Sequence variation in the virion spike is thought to occur in response to rapidly changing host receptor epitopes that might block or inhibit the entry of viral pathogens, resulting in what is known as the virus-host "arms race" [93].

The 5-fold axis interaction is not unlike a ball-and-socket joint, such that a closed fist-shaped region located in the upper left portion of the capsid protein, just below the tri-fold loop protrusion, interacts with a saddle-shaped socket located in the right underside of the MCP. The fist-shaped protrusion is oriented so that it both fills the socket, and is held fixed in a slightly angled position. Five MCPs interlink in this way to form a slightly convex pentamer. These form the twelve pentameric faces of the complete icosahedral virus particle. An interlocking tessellation of 60 MCPs at the 2-fold, 3-fold and 5-fold interaction interfaces allows for assembly of the mature virus particle [80] (Figure 2.4).

Homology modeling of Gokushovirinae, Pichovirinae and Alpavirinae capsid proteins

A subset of 17 sequences, representing each of the MCP clades as determined from the N-J tree (Figure 2.1), were selected for the construction of homology models. The sequences selected for modeling were chosen to represent a broad range of sequence diversity while utilizing a minimum number of taxa. Each structure model is generated based on its predicted homology to the cryo-EM structure of the SpV-4 capsid protein using the CPH Model server (see Methods section). The CHP Model Server utilizes a threading algorithm which selects the most favorable solved structure from the Protein Data Bank upon which to construct a three-dimensional hypothetical protein model for a 
given amino acid sequence query. Each input sequence is first evaluated on the basis of its amino acid sequence similarity to solved structures in the Protein Data Bank (www.PDB.org). Secondary structures are predicted from the input sequence data, which must also match the reference PDB file before threading commences. A homology model will only be generated based on a reference structure if these initial criteria are met. Additionally, a "Z-score" is also calculated for successfully generated models, indicating the reliability of the predicted models. All homology models generated for this analysis yielded $Z$-score values substantially exceeding the minimum confidence value $(Z=10)$.

When the 17 structure homology models of MCPs from the Gokushovirinae, Pichovirinae and Alpavirinae groups (including SpV-4) are structurally aligned, the remarkable dichotomy between structural congruency and amino acid sequence heterogeneity can be clearly seen (Figure 2.5). Coloring the models by RMSD illustrates a potentially high degree of predicted structural congruency among all of the proteins, and that structural variability is mainly confined to insertion loops at the junctions between strands and helices.

Coloring the models according to the BLOSUM 80 substitution matrix score shows that while there is a low level of sequence identity among all of the proteins, individual residues are most often substituted with amino acids with similar structural or chemical characteristics. However, when viewing based on sequence identity within conformational space, very few amino acids are identical in all of the models (Figure 2.5). 
The maintenance of structural congruency among these proteins is striking, considering the level of amino acid sequence plasticity observed in the Microviridae family of MCPs. These proteins must not only interact with one another in highly precise ways along at least three separate interfaces in order to assemble into a structurally viable particle, and the mechanism(s) that allows for both host recognition and entry must also remain intact. The major capsid proteins of these viruses are able to sustain significant sequence variability, while maintaining several structural features required for proper assembly, and functional capabilities required for viability.

\section{Identifying highly conserved regions within the microvirus major capsid protein}

A conservative hypothesis for how this balance between sequence divergence and structural homology is maintained, is that a high degree of sequence variation can be tolerated as long as functional attributes and protein-protein interfaces required for proper assembly of the virion remain intact. Thus, one goal of this investigation is to determine whether the protein-protein interfaces that are required for these interactions are preferentially conserved in order to maintain steric and chemical compatibly at each of the binding surfaces.

First, conserved amino acid residues are revealed through a SpV-4 structure-based sequence alignment of $17 \mathrm{MCP}$ amino acid sequences representing the three main clades (Supplemental Figure S-2.1). To refine this alignment in such a way as to indicate the spatial positions of the conserved amino acids, the 17 structure homology models were 
structurally aligned to show how the conserved residues are configured in conformational space (Figure 2.6) The structural alignment indicates that there are 13 spatially identical amino acids among all 17 models used in this analysis, most of which appear to be positioned in the 2-, 3-, and 5-fold interaction interface regions. Reducing the scope of the alignment to the 11 Gokusho- and 5 Pichovirinae proteins, indicates that there are several additional amino acids that are identical within these subgroups, which in some instances appear to be associated with virion assembly, but several of these conserved residues are positioned at the base of the $\alpha$-helix which supports the tri-fold loop. Narrowing the scope of the alignment even further to the group of 11 Gokushovirinae proteins shows that the conserved residues predominantly lie along a diagonal core region of the MCP, at the base of the tri-fold loop $\alpha$-helix and spanning the region between the 5-fold axis protrusion and the 2-fold axis interface. Conservation within these particular regions may indicate their importance in maintaining assembly interactions, stabilizing the structural integrity of the MCP, and may perhaps be associated with important functional properties of the protein as well.

In order to ascertain which of the 14 universally conserved amino acids are likely associated with assembly interfaces, molecular models of BSL-1436 are drawn in a space-filling mode so that the highlighted conserved residues exposed near or at the surface of the protein may be seen (Figure 2.7). This process demonstrates that 11 out of the 14 spatially conserved amino acids appear to be primarily exposed at the 2- and 3fold interaction interfaces, and are thus likely to be necessary for proper assembly of the virion. 
By superimposing the BSL-1436 structure onto SpV-4 MCPs in the biological assembly file, oriented in such a way as to show the 2-, 3- and 5-fold interactions between subunits, it appears that many of the highly conserved residues in the MCP are very likely to be involved in inter-subunit binding interactions (Figure 2.8). The 2-fold interface seems to be largely controlled by interactions between Arg-90 and Tyr-308 occurring between the two MCPs. The other conserved residues surrounding Arg-90 (Pro-88, Glu-380 and perhaps Pro-53) appear to associate with the region defined by the conserved triad of Asp-411, Pro-415 and Gln-423 on the 5-fold axis "ball” protrusion. This conserved D411-P415-Q 423 triad region might also interact with the region harboring the conserved Arg-289 of the tri-fold loop $\alpha$-helix, at the junction of the 3-fold and 2-fold interactions. Surprisingly, the D411-P415-Q 423 triad of the "ball" protrusion does not actually appear to be involved in the 5-fold axis interaction. Instead, it would appear that the non-conserved residues, forming the rest of the "ball" structure, neatly line the inner surface of the saddle-shaped "socket" on the underside of the MCP (roughly encompassing the region between the conserved Pro-45 and Pro-53 residues). Thus, this assessment appears to indicate that amino acid sequence conservation is not as critical for maintaining interactions between MCPs at the 5-fold axis interface.

A rather striking and unexpected feature revealed through the analysis of structure-based sequence conservation is that many of the highly conserved residues neither appear to be specifically involved in MCP assembly interactions, nor positioned in regions that might help stabilize the internal secondary and tertiary structure of each MCP. In fact, even when viewing only the residues conserved within the clade of 
Gokushovirinae, very few amino acids are conserved within the $\beta$-barrel or $\alpha$-helices comprising the structural core of the MCP (Figure 2.8). This is somewhat surprising, as homology modeling indicates that the highest degree of structural congruency is maintained within the core or backbone of these secondary structures within the protein (Figure 2.5). Instead, the conserved residues are mainly arranged in an I-beam-like configuration extending perpendicularly across the 2 -fold axis of symmetry, spanning the entire length of each MCP dimer between the "ball" protrusions of each subunit (Figure 2.8) This double band of conserved residues extending across each dimeric unit may help to stabilize the superstructure of the virion somehow, however, the true significance of this pattern of conservation is a mystery.

Investigating potential capsid protein adaptations that are unique to BSL microviruses

Successful adaptation to a hot and highly acidic environment undoubtedly involves many different factors. Amino acid substitutions that stabilize protein-protein interactions involved in the assembly of viable virus particles are likely to be necessary as a virus adapts to a new physiochemical environment. However, structure-based sequence analysis alone is insufficient to prove whether a particular substitution or set of mutations is the direct result of positive selection. This approach is, however, quite useful for determining which residues have been conserved, and are thus likely to be vital in some way for proper assembly and functioning of the mature virion. Structure-based sequence alignments, while useful in some regards, do not reveal other facets of evolution and adaptation that may be relevant to different environmental conditions. Without direct 
experimentation in the lab, it is not possible to definitively determine the role each of the conserved residues plays in terms of virion viability.

Thus, in an attempt to identify other adaptive mechanisms that pertain specifically to viruses in the hot, acidic environment of BSL, the amino acid sequences of all viral capsid proteins shown in the N-J tree phylogeny were individually uploaded to the ExPASy ProtParam tool for analysis (see Methods section). This tool was used to calculate the following physiochemical characteristics: theoretical isoelectric point (pI), the number of negatively and positively charged amino acids, the aliphatic index, and the grand average of hydropathicity (GRAVY). Calculating these physiochemical attributes of individual capsid protein sequences revealed that the gokushovirus-like sequences isolated from BSL exhibit a relatively high isoelectric point and relatively low hydropathicity (i.e., they are somewhat less hydrophobic) by comparison to other related proteins found in different environments (Table 2.2).

A trend in either direction, with respect to pI and GRAVY, may be linked to whether the virus originates from a freshwater or marine environment. The most pronounced effect in this regard is observed in viruses specific to BSL, in that they have the highest isoelectric points and lowest hydropathicity scores relative to all of the other MCPs. The only other protein with a high $\mathrm{pI}$ is the $\mathrm{SpV}-4 \mathrm{MCP}$. The first $37 \mathrm{~N}$-terminal amino acids of the SpV-4 MCP sequence, however, are not present in the SpV-4 model $(1 \mathrm{KVP})$, indicating that they are either not expressed, or cleaved off. If they are expressed as part of the protein, as the GenBank sequence file (NP_598320.1) appears to indicate, these N-terminal amino acids, being largely positively charged, possibly interact with the 
nucleic acid packaged within the virion. Moreover, the SpV-4 MCP GRAVY score calculated based on the complete ORF, is quite high by comparison to the BSL proteins. In light of these factors, the pI and GRAVY score of the SpV-4 MCP protein was also calculated after removal of the $37 \mathrm{~N}$-terminal residues that are not part of the cryo-EM model, which resulted in a dramatic reduction of the SpV-4 $\Delta \mathrm{pI}$ to 6.53 , while leaving the hydropathicity score relatively unaltered.

One possible explanation for the high isoelectric points observed in the BSL MCPs may be that it ensures that the virion maintains a strong positive surface charge in the acidic environment, which could result in increased binding affinity of the virion to aluminosilicate clay particles or organic substrates suspended in the BSL water column and sediment [2]. Clay minerals have long been used to extract viruses from water sources for purification purposes, by exploiting the strong binding affinity between proteins and clay particles $[94,95]$. If viruses in BSL are actually binding to clay particles, it may allow them to maintain a closer proximity to their host organisms in microbial mats, or to lithotrophic hosts associated with sulfur- and iron-based sediments, and thus increase the likelihood of infection. However, there are many complex factors affecting adsorption of virus particles by different types of soils and sediments; each variable, in turn, being dependent upon various $\mathrm{pH}$ levels and types of solutes present, etc., [96-99]. Without directly studying the adherence of native viral and microbial species to BSL sediment, there is no way to conclusively state whether (or how) a high capsid protein isoelectric point might provide a survival advantage to the virus. 
Isoelectric points are calculated based on the amino acid sequence of the protein, and does not take into account the position of each amino acid within the tertiary structure. If a PDB structure file is available, solvent accessibility (based partially on amino acid isoelectric point) can be mapped to the surface of the protein in question using the PDB2PQR algorithm (see Methods section) (www.poissonboltzmann.org/pdb2pqr). This process generates an image of the model showing regions of high and low solvent accessibility on the surface of the protein (Figure 2.9). The surface of the BSL-1436 capsid protein that faces the interior of the virion, where the viral DNA is packaged, appears to be much less accessible to solvent than is the marine SARss phi-2. The saddle-shaped "socket" of the BSL-1436 5-fold interaction interface also appears to be much less accessible to solvent than the SARss phi-2 capsid protein interface. When the "ball" protrusion binds the saddle-shaped "socket" at the 5-fold axis of symmetry, it would appear that the resulting interface between BSL-1436 subunits would be highly inaccessible to solvent. In this regard, decreased solvent accessibility at the assembly interfaces might result in the formation of much tighter junctions between each subunit, which may, in turn, help protect the viral DNA packaged inside the virion from acid hydrolysis. Another possibility is that decreased solvent accessibility might inhibit denaturation of the virion's quaternary structure as it is subjected to the highly acidic environment of BSL.

New observations such as this have been made possible only through structure homology modeling of metavirome sequence data. The wealth of information provided by structural studies and metagenomic surveys provide fertile ground for the formulation 
of new, testable hypotheses that may greatly expand our understanding of how viruses evolve and adapt to different environments and ecosystems.

\section{MATERIALS AND METHODS}

Microviridae sequences utilized in the sequence and structure analyses:

Several Gokusho- and Pichovirinae sequences from the freshwater lakes, Pavin and Bourget were obtained from Roux et. al. [44, 45], Pichovirinae prophage sequences, originally found within the genomes of their Bacteroidetes hosts, were first identified by Krupovic et. al. [45, 77], and were formerly referred to as "Bacteroidetes-associated Microvirus-related provirus" or BMV. Pichovirinae sequences used as part of this analysis were obtained from the Dryad Digital Repository prepared by Roux et. al., cited above. All GenBank accession numbers, and references to the Roux sequence database, associated with the sequences used in this analysis are listed in Table 2.1.

Two Boiling Springs Lake gokushovirus contigs were detected in the BSL metavirome data; contig-1436 and contig-1013. Each contig appeared to represent a different strain of virus due to sequence heterogeneity between them, but both were identified as gokushoviruses by BLAST comparisons of predicted open reading frame segments derived from the contigs. The complete circular genomes of both 1436 and 1013 gokushoviruses were amplified by inverse PCR from the $\varphi 29$-amplified metagenomic DNA. Phire polymerase (Finnzymes) was used to amplify both genomes 
from $10 \mathrm{ng}$ of BSL metagenomic DNA template using the following inverse PCR primer sets designed based on the contig sequences; BSL-1436: 5'-

TAATTACACGTTGTTGCGCCGTG-3' \& 5'-GTTGAAGCCGACGCATCAGGAC-3' and BSL-1013: 5'-TTATTCAGCCGCCTTTTCAGG-3' \& 5'-

GCCGTAGCACAGTTACCTTAC-3'. The following inverse PCR reaction conditions were used: $98^{\circ} \mathrm{C}, 30$ sec., $30 x\left[98^{\circ} \mathrm{C}-5\right.$ sec., $58^{\circ} \mathrm{C}-5$ sec., $\left.72^{\circ} \mathrm{C}-2 \mathrm{~min}.\right], 72^{\circ} \mathrm{C}-2$ min. PCR amplicons were purified to remove dNTPs, primers and enzyme, and the amplicons were used as templates for Big Dye Terminator (BDT) sequencing confirmation. The resulting sequences, produced incrementally by primer "walking" along the length of each genome, were assembled using the original BSL contigs as scaffolds. Any errors in the original contig sequences were corrected using the BDT sequences as a reference.

Twelve environmental microvirus capsid protein sequences were selected for comparison to the two Boiling Springs Lake metagenome-derived MCPs BSL-1436 and BSL-1013. Ten additional well-characterized laboratory isolates were selected from GenBank to guide the sequence and structure-based analyses (Table 2.1). These 24 sequences, encompassing the Gokushovirinae, Pichovirinae and Alpavirinae microvirus subgroups, were specifically chosen in order to represent a broad range of sequence diversity from a wide variety of physiochemical environments in which microviruses have been found, while using a minimal set of representative sequences. All of these sequences were compared to the Spiroplasma phage-4 (SpV-4) major capsid protein gene (M17988.1, GI:334998, NP_598320.1) because its structure has been solved by cryoelectron microscopy (cryo-EM) and is available in the Protein Data Bank, www.pdb.org, 
as PDB ID\#: 1KVP [80]. The structural evolution of the BSL-1436 and BSL-1013 MCPs, as well as those from an additional 22 metagenomic sequences and laboratory isolates, are evaluated on the basis of the $\mathrm{SpV}-4$ sequence and structure.

\section{BLAST analysis of microvirus sequences:}

Each of the major capsid protein gene nucleotide and amino acid sequences were compared by tBLASTn and BLASTp to the Spiroplasma phage-4 major capsid protein gene, respectively, using BLAST2seq to generate E-values (Table 2.1). The best E-value resulting from each search was listed for comparison, and the data table was sorted according to the best amino acid sequence comparison E-value. Percent identity values between the $\mathrm{SpV}-4$ reference and query amino acid sequences were roughly estimated for each pair-wise comparison by Needleman-Wunsch global alignment [100]. NeedlemanWunsch global alignment estimation of percent identity presumes adequate sequence homology exists between the query and reference sequences. As the SpV-4 MCP reference and query MCP DNA sequences are too divergent to be aligned, percent nucleotide identity calculations using global alignments are not valid, and are therefore not included.

\section{Phylogenetic analysis of microvirus sequences:}

A CLUSTALW amino acid sequence alignment was performed on a the complete set of 27 capsid protein amino acid sequences, encompassing the Gokushovirinae, 
Pichovirinae and Alpavirinae subgroups using MEGA5 [101]. MEGA5 was then used to produce a Neighbor-Joining $(\mathrm{N}-\mathrm{J})$ tree from the alignment, rooted by the Enterobacteria phage $\alpha 3$ and $\varphi X 174$ microvirus MCPs (Figure 2.1) [102]. The evolutionary distances were computed using the Poisson correction method in MEGA5 [103].

Generating structure homology models of BSL and other capsid protein sequences: A subset of 17 sequences derived from the N-J tree were selected for the construction of homology models based on the solved structure of the SpV-4 capsid protein, 1KVP. The CPH Model Server (http://www.cbs.dtu.dk/services/CPHmodels/) [104] was used to generate PDB files of the following capsid protein sequences: Spiroplasma Phage-4 (SpV-4), BSL-1436, BSL-1013, GOS-11182, Gokusho-Bourget 164, phiCPG1, Chlamydia Phage-3 (CHP3), SARss phi-1, SARss phi-2, phiMH2K, Gokusho-Human Gut, Picho-JCVI 003, Picho-Pavin 279, Picho-coral 59, Pichomicrobialite 66-001, Picho-Bourget 523 and Alpa-CF7ML Human Lung. The structure for the $\mathrm{SpV}-4$ capsid protein was generated by uploading the $\mathrm{SpV}-4$ capsid protein sequence from GenBank (NP_598320.1) to the CHP Model server for threading to its own PDB file (1KVP), as it was discovered during the course of this analysis that the $1 \mathrm{KVP}$ structure file erroneously includes the amino acid sequence for the $\varphi \mathrm{X} 174$ capsid protein. This resulted in the formation of a new PDB model for $\mathrm{SpV}-4$ that contains the correct amino acid sequence for use in structure-based sequence analyses. 
Structural evaluation of the gokushovirus capsid protein monomers:

To determine regions of amino acid sequence conservation within the group of 17 MCPs chosen for detailed structural analysis, a structure-guided sequence alignment was conducted based on the SpV-4 PDB file using PROMALS3D [105] (Supplemental Figure S-2.1). To illustrate how sequence conservation is spatially oriented within the folded tertiary configuration of the proteins, the PDB models generated by CPH Model server were structurally aligned using the "STAMP structure alignment" tool in the VMD MultiSeq software package [106], after performing a CLUSTAL sequence alignment of the amino acid sequences embedded in the PDB files. (Figures 2.5, 2.6, 2.7 and 2.8).

Illustrating virion axes of symmetry and the assembly of MCPs:

Images of the BSL-1436 major capsid protein homology model, rendered in VMD, were arranged by hand in PowerPoint to illustrate the protein-protein interactions that result in the formation of the 2-fold, 3 -fold and 5-fold axes of symmetry within the complete virion (Figure 2.3).

Generating the biological assembly file of a BSL-1436 MCP trimer showing 2-, 3- and 5fold axes of symmetry:

To illustrate the conserved amino acid residues involved in 2-fold and 5-fold monomer self-assembly interactions, a three-monomer PDB file of the BSL-1436 MCP was scripted by hand based on the $1 \mathrm{KVP}$ biological assembly file (1KVP.pdb1.gz). First, three of the $60 \mathrm{SpV}-4 \mathrm{MCP}$ subunits, adjoined at the appropriate axes, were copied from 
the $1 \mathrm{KVP}$ biological assembly file and pasted to the 1KVP.pdb file. Each of the three MCPs were assigned a new chain designation to make them visible in the VMD display. Models of the BSL-1436 MCP were then structurally aligned to all three SpV-4 MCPs using STAMP structural alignment in order to adjust their spatial coordinates to match the $\mathrm{SpV}-4$ subunit orientations in the original 1KVP biological assembly. The corrected $\mathrm{SpV}-4$ file, containing the true $\mathrm{SpV}-4$ sequence, was then overlaid onto the assembly, along with the 16 other MCP models used as part of the analysis. All structures were first aligned by CLUSTAL before performing a spatial alignment using STAMP. Conserved amino acid sequences could then be highlighted based on the STAMP structure alignment. Spatially conserved residues could then be superimposed on the BSL-1436 frames in "VDW" mode as needed (Figure 2.8)

\section{Calculating physiochemical profiles of microvirus capsid proteins:}

Twenty-three microvirus capsid protein amino acid sequences, including the two BSL isolates, were uploaded individually to the ExPASy ProtParam tool (http://web.expasy.org/protparam/) [107] for analysis. This tool was used to calculate; theoretical isoelectric point (pI), the number of negatively and positively charged amino acids, the aliphatic index, and the grand average of hydropathicity (GRAVY). The output data were downloaded and complied into an Excel spreadsheet and sorted according to pI. An additional SpV-4 $\Delta$ sequence was created and added to this data, by removing the first $37 \mathrm{~N}$-terminal amino acids of the SpV-4 sequence that are not present in the SpV-4 model 1KVP (Table 2.2). 
Generating solvent accessibility PQR models:

The homology model PDB files for BSL-1436 and SARss phi-2 were uploaded to the PDB2PQR model generator (http://nbcr-222.ucsd.edu/pdb2pqr_1.8/)[108, 109] to map solvent accessibility to the tertiary structures of the MCPs. Solvent accessibility images of the protein models are generated automatically by PDB2PQR by applying default settings, and are based on Poisson-Boltzmann electrostatic calculations that have been mapped to the surface of the protein (Figure 2.9).

\section{SUMMARY}

As the Microviridae appear to be one of the most globally ubiquitous and diverse virus families $[45,51,110]$, they offer an exceptional model for studying the structural dynamics of viral protein evolution and adaptation. Particular regions of microvirus major capsid proteins appear to be influenced by opposing forces of positive selection, acting on the putative host receptor-binding region, and purifying selection, acting on the protein-protein interaction interfaces involved in assembly of the virion. However, since the MCP sequences are poorly conserved overall, a structure-based analysis approach was implemented in order to observe variations in the MCPs within this highly diverse group of viruses. Structure homology modeling of Microviridae capsid proteins was carried out to determine the specific regions of the protein that are conserved, thus highlighting the residues that are most likely to be vital for viability of the virion. 
A cladogram produced as the result of aligning the set of Microviridae MCP amino acid sequences indicates that the two BSL MCPs, 1436 and 1013, are closely related to one another and group with another gokushovirus from a freshwater source. This group of freshwater virus MCPs group together within a larger clade of Gokushovirinae that have been detected in both marine and freshwater environments, indicating a lineage of environmental gokushoviruses that is distinct from isolates that are often associated with human and animal samples (Figure 2.1).

Homology modeling based on the cryo-EM structure of the Spiroplasma phage-4 $(\mathrm{SpV}-4)$ capsid protein indicates that all Microviridae capsid proteins are likely to be structurally congruent, despite substantial genetic and amino acid sequence heterogeneity within the group (Figure 2.5). A structure-based sequence alignment of Gokusho-, Pichoand Alpavirinae capsid protein taxa suggests that the residues affected most acutely by purifying selection appear to be associated with inter-subunit interactions associated with assembly or stabilization of the virion. Based on this analysis, it is assumed that the 2and 3-fold interfaces are the most crucial for assembly, as no putative interactions between exposed, conserved amino acids could be detected specifically at the 5-fold axis of symmetry. Moreover, there appears to be a combined interaction at the junction of the 2-, 3- and 5-fold interfaces, in a region at the base of the tri-fold loop $\alpha$-helix, between 3 MCPs that appears to be important for assembly (Figure 2.8).

Spatial patterns of amino acid sequence conservation also reveal that many of the most highly conserved residues are generally not associated with the core secondary structure elements of the MCP that are otherwise predicted by modeling to be highly 
structurally conserved (Figures 2.3 and 2.8). Instead, the most well-conserved residues form banded "girder"-like structures which laterally extend along the entire length of MCP dimers within the virion. Thus, somewhat surprisingly, sequence conservation does not appear to be strongly correlated with structural conservation or congruency in this group of proteins (Figure 2.8).

In addition to amino acid conservation at the putative 2- and 3-fold symmetry interfaces, global physiochemical attributes of the capsid proteins indicate that adaptation to the hot, acidic environment of BSL may occur to a certain extent through an overall increase in protein isoelectric point (pI), as analysis predicts that the pIs of BSL capsid proteins are markedly higher than that of other microviruses (Table 2.2). The specific reason for the marked difference between the BSL capsid protein isoelectric point relative to the others is unknown.

The lack of solvent accessibility at the 5-fold axis interface and the interior surface of the capsid protein, as observed through APBS (PQR) modeling, indicates that the BSL-1436 virion, once fully assembled, may be less permeable to solvent than the marine SARss phi-2 virion (Figure 2.9). This adaptation might allow the BSL-1436 virus to more tightly encapsulate the viral DNA, thus protecting it from acid hydrolysis. However, it might also increase the substrate binding affinity of the virus to sediment, or conversely, increase its solubility, or even help keep the free virion from denaturing in the highly acidic water of BSL once released by the infected cell. All of these hypotheses could be tested once a suitable host is found, and it becomes possible to cultivate and study these viruses in vitro. A worthwhile research endeavor might also involve 
evaluating all capsid protein sequences detected within BSL and other acidic

environments, to assess whether increased $\mathrm{pI}$ is an ecologically relevant adaptation common to all viruses inhabiting acidic environments. 


\section{CHAPTER 2 FIGURES:}

Figure 2.1 Evolutionary relationships of microvirus capsid proteins

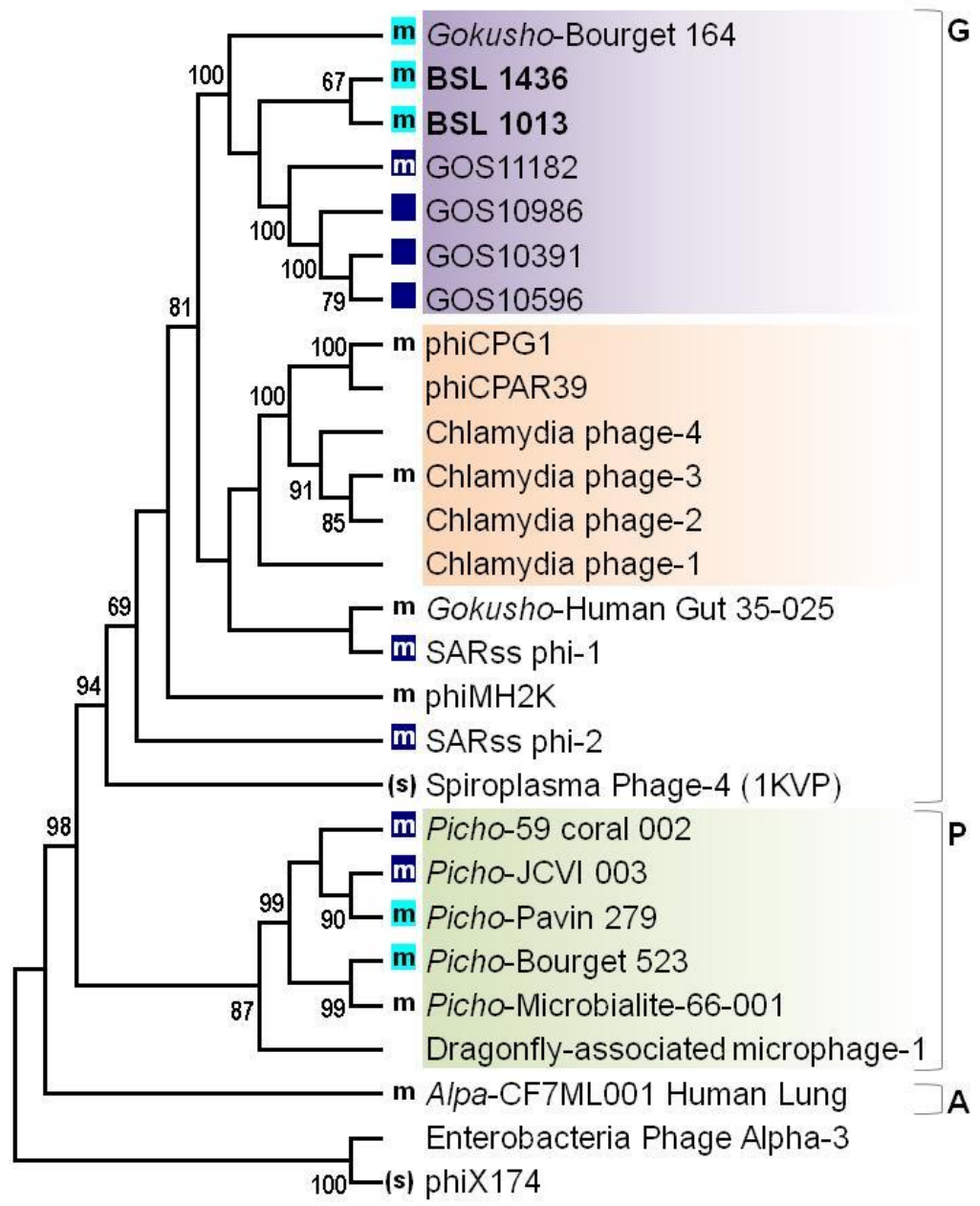

Evolutionary relationships among the Gokushovirinae are inferred using the NeighborJoining method on an alignment of the MCP proteins (Supplemental Figure S-2.1). The optimal tree with the sum of branch length $=7.43$ is shown. The percentage of replicate trees in which the associated taxa clustered together in the bootstrap test (with 1000 replicates) are shown next to the branches [111]. Bootstrap values greater than $60 \%$ are shown. Viruses isolated from freshwater sites are indicated with a light blue square, and viruses from marine environments are indicated by a navy blue square. An " $m$ " to the left of the taxon name indicates that a structure homology model has been generated for the sequence, and an "(s)" indicates that the structure has been experimentally determined by cryoEM or crystallography. All homology models are predicted based on the Spiroplasma Phage-4 strucutre, 1KVP. Gokushovirinae are bracketed and labeled "G", Pichovirinae are labeled "P" and Alpavirinae is labeled "A". The "BSL / environmental clade" is shaded violet, the "Chlamydia phage clade" is shaded orange, and the "Pichovirinae clade" is shaded green. 
Figure 2.2 Homology model of the BSL-1436 capsid protein threaded to SpV-4 $1 \mathrm{KPV}$

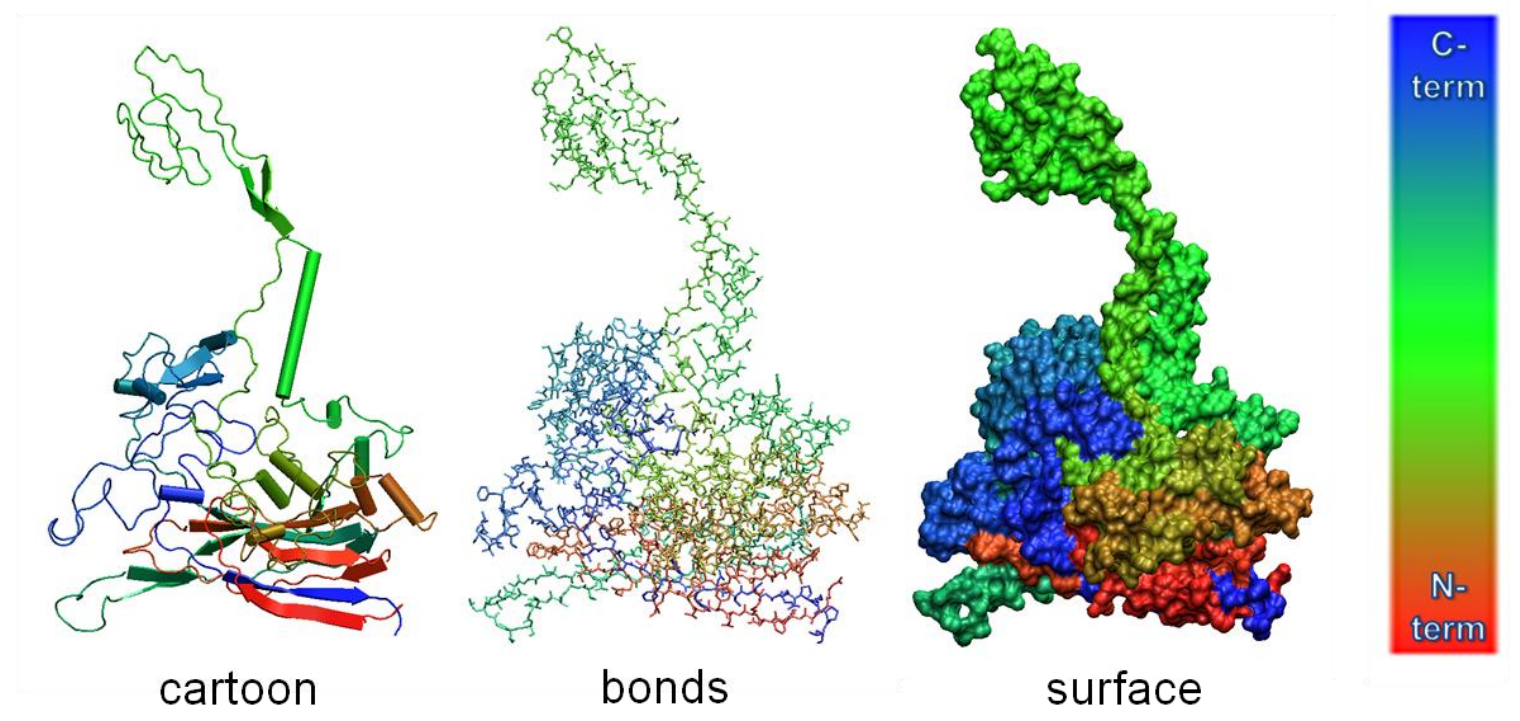

Models for the BSL-1436 MCP generated in VMD are colored by RGB "Index", with red indicating the $\mathrm{N}$-terminus, blue indicating the $\mathrm{C}$-terminus, and green indicating the central portion of the protein sequence. The "Cartoon" mode shows the amino acid backbone or alpha carbons with $\alpha$-helices as rods and $\beta$-strands as arrows. "Bonds" mode shows the spatial orientation of each amino acid, and "Surface" mode is an atomic spacefill rendering of the amino acids within the model. 
Figure 2.3 Gokushovirus capsid protein assembly axes

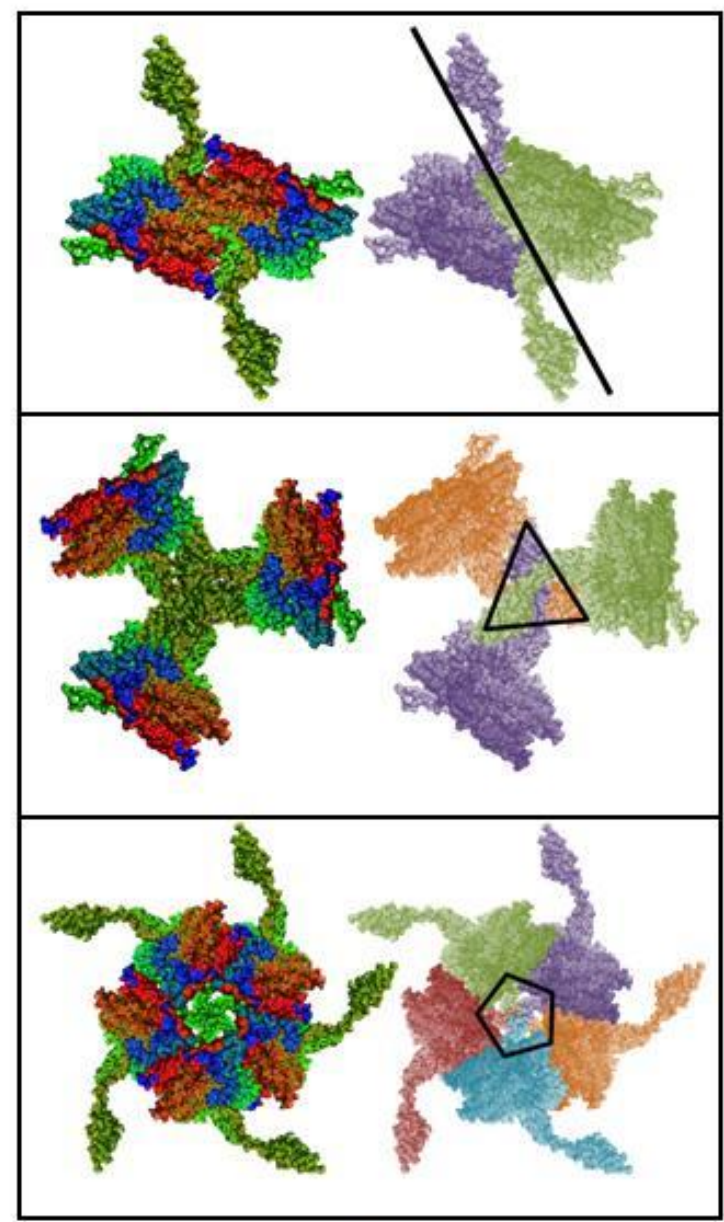

Two-dimensional images of the BSL$1436 \mathrm{MCP}$ model arranged by hand to indicate the protein-protein interactions that result in the formation of the 2-fold, 3 -fold and 5-fold axes of symmetry of the complete virion. On the left, MCPs illustrated in surface mode are colored by "Index" and by whole subunit to the right, to visually discern each monomer in its quaternary context. An assembly of three MCPs, modeled on the biological assembly file of the SpV-4 (1KVP.gz), shows three subunits, colored by whole subunit, interacting at all symmetry axes.

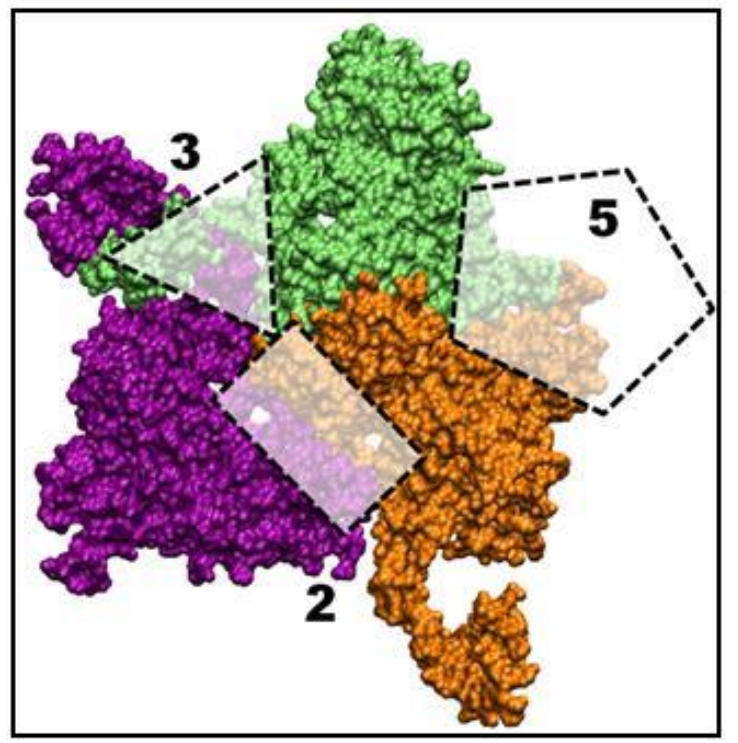



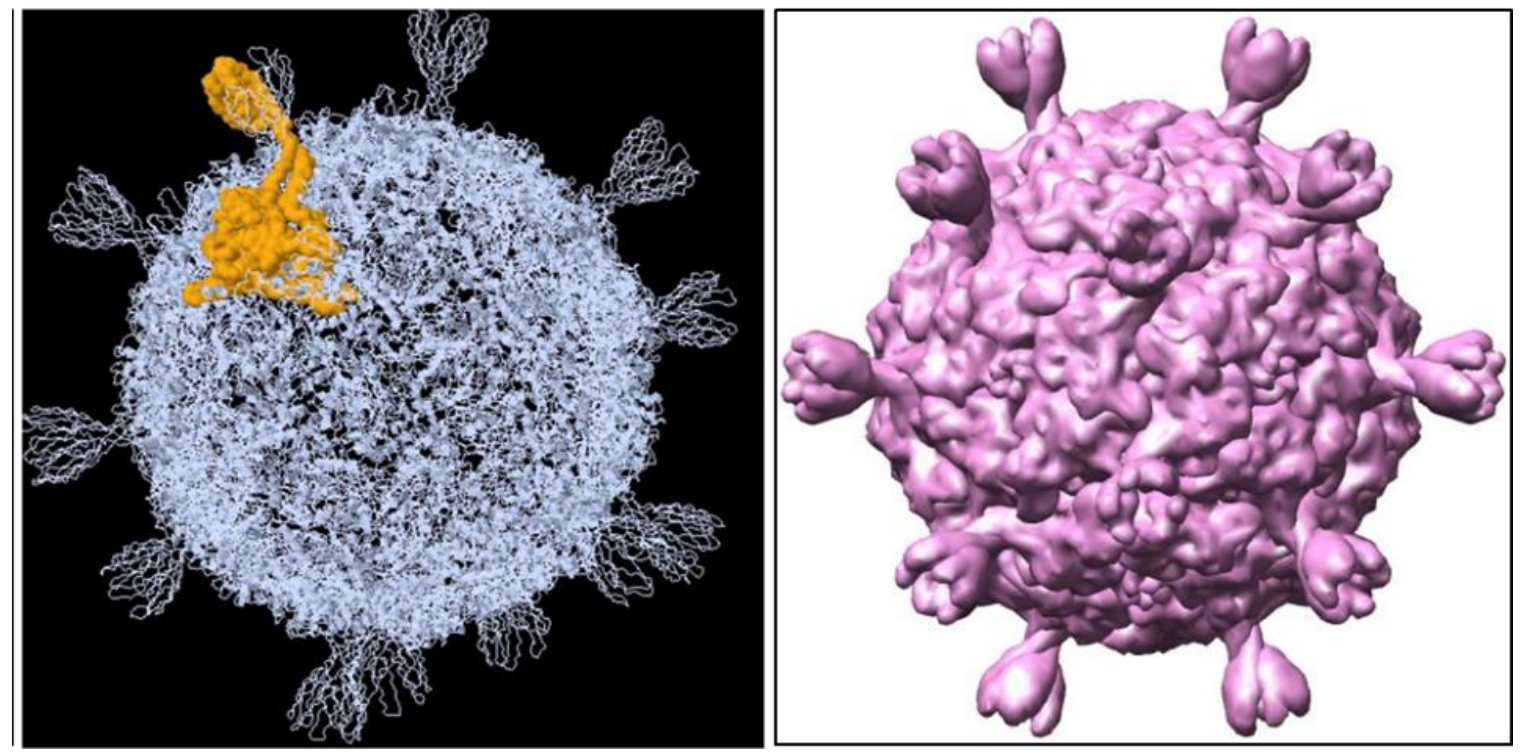

Sixty MCPs assemble to form an icosahedral virus particle. An interlocking tessellation of capsid protein monomers at the 2-fold, 3-fold and 5-fold interaction interfaces result in the formation of the mature virus particle. The panel on the left shows a single MCP, highlighted in "surface" mode and colored in orange, as it is positioned within the complete SpV-4 virion. The far right panel shows the complete SpV-4 icosahedral 60mer virion (image obtained from www.PDB.org, PDB ID\# 1KVP). 
Figure 2.5 Structural alignment of 17 Microviridae MCPs

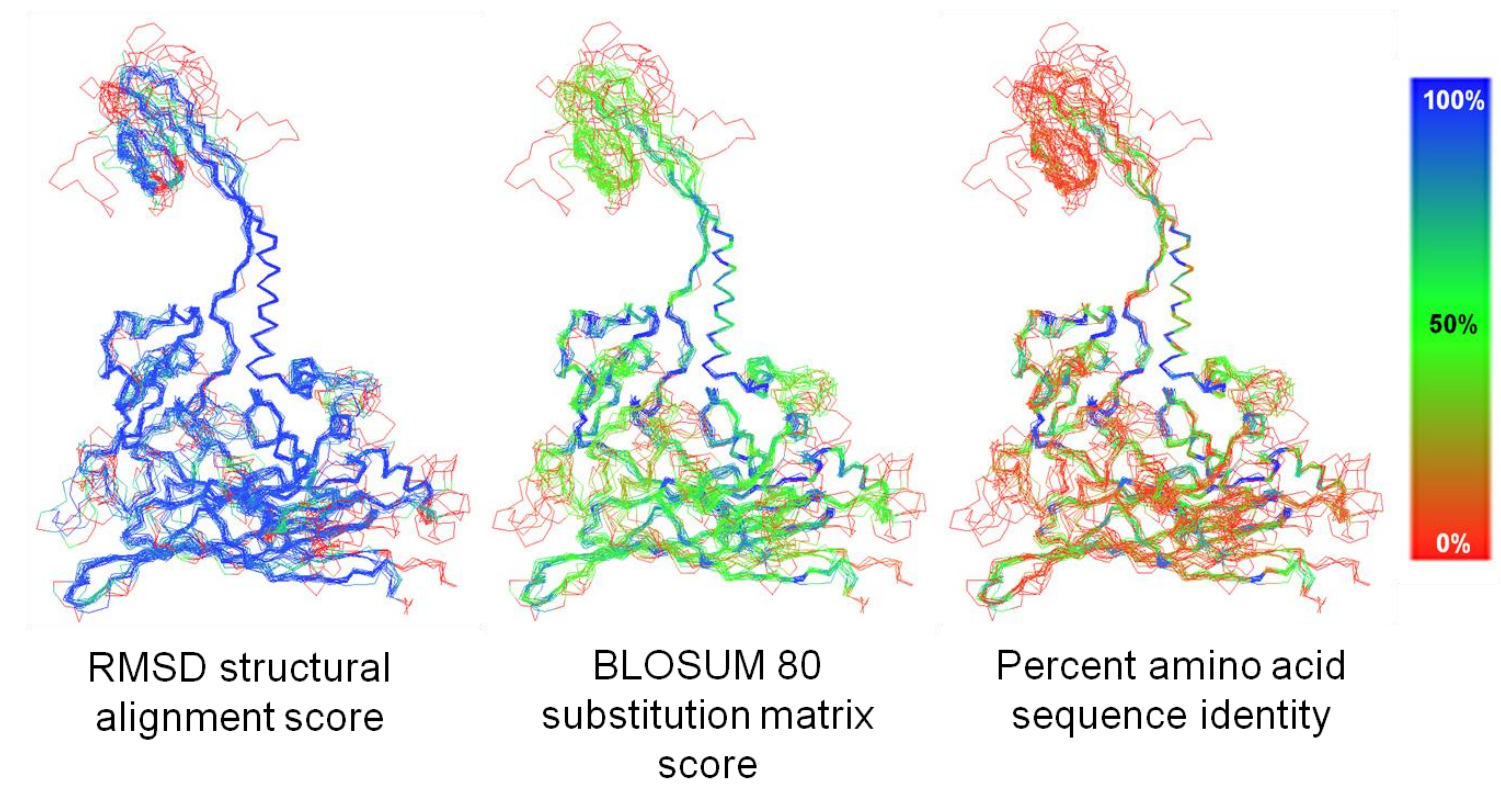

Homology models of 17 MCPs are structurally aligned to the cryo-EM structure of SpV4 (PDB ID\# 1KVP) in VMD. Each backbone atom wireframe model is colored according to RMSD, a measure of how well each structure fits within the spatial configuration of SpV-4, BLOSUM 80, a measure of amino acid chemical similarity at each position relative to $\mathrm{SpV}-4$, and percent amino acid sequence identity, which represents the average co-occurrence of an identical residue in a given consensus position. The scale, from 0 to $100 \%$ indicates congruency with respect to each of the parameters shown. Wireframe models shown are: Spiroplasma phage-4 (SpV-4), BSL-1436, BSL-1013, GOS-11182, Gokusho-Bourget 164, phiCPG1, Chlamydia Phage-3 (CHP3), SARss phi-1, SARss phi2, phiMH2K, Gokusho-Human Gut, Picho-JCVI 003, Picho-Pavin 279, Picho-coral 59, Picho-microbialite 66-001, Picho-Bourget 523 and Alpa-CF7ML Human Lung. 
Figure 2.6 Spatial conservation of amino acids in CP model structures among three Microviridae clades
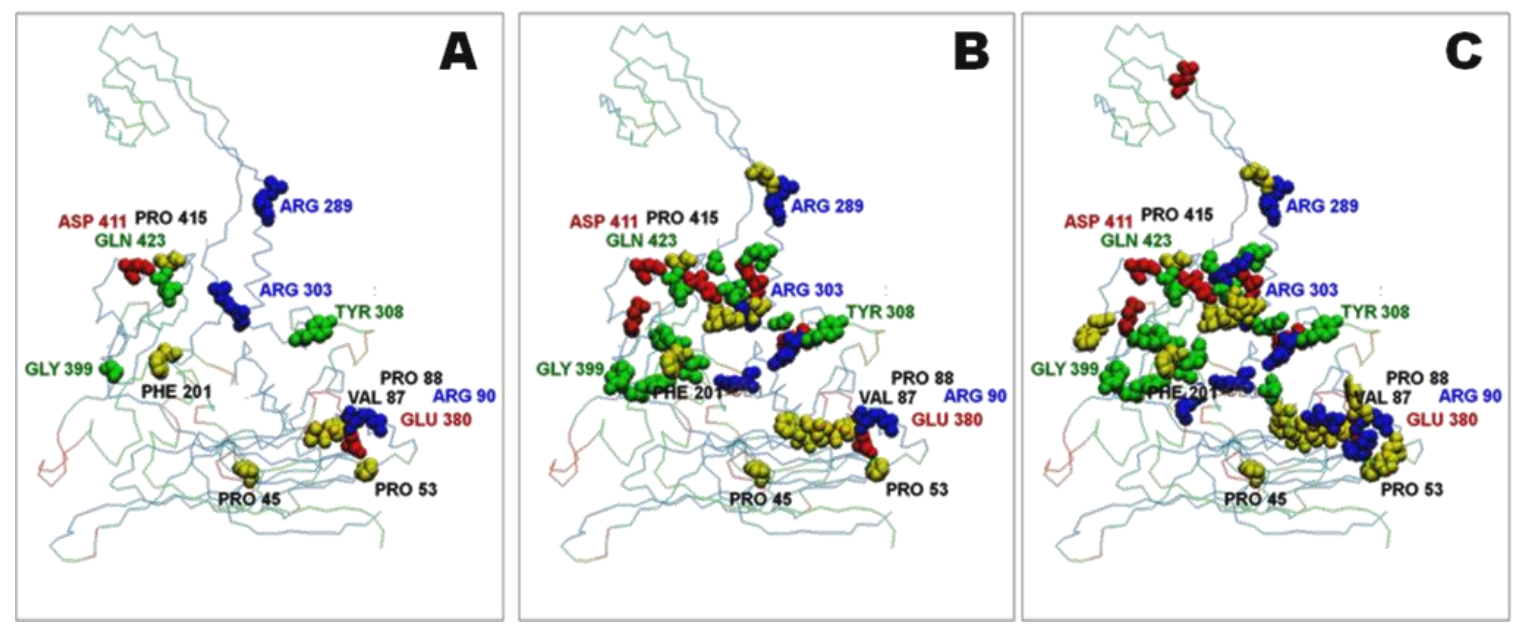

The BSL-1436 wireframe model of the backbone atoms is shown. Superimposed upon the wireframe model are amino acids (shown in "VDW" mode) that are spatially conserved in tertiary structure-space. Panel A) shows the identical residues conserved among Gokusho- (11 taxa), Picho- (5 taxa), and Alpavirinae (1 taxon) MCPs. Panel B) shows identical residues in the Gokusho- (11) and Picho- (5) clades, and panel C) depicts the identical amino acid residues in the Gokusho- (11) group only. Residues shown in the models are colored by "Residue Type"; polar = green, non-polar = yellow, acidic $=$ red and basic $=$ blue. Only residues that are universally conserved among all 17 sequences are labeled and numbered according to the BSL-1436 position in all diagrams, while additional residues that are conserved within narrower subsets of taxa are shown, but are not labeled. 
Figure 2.7 Spatially conserved amino acids partially exposed on the surface of the MCP
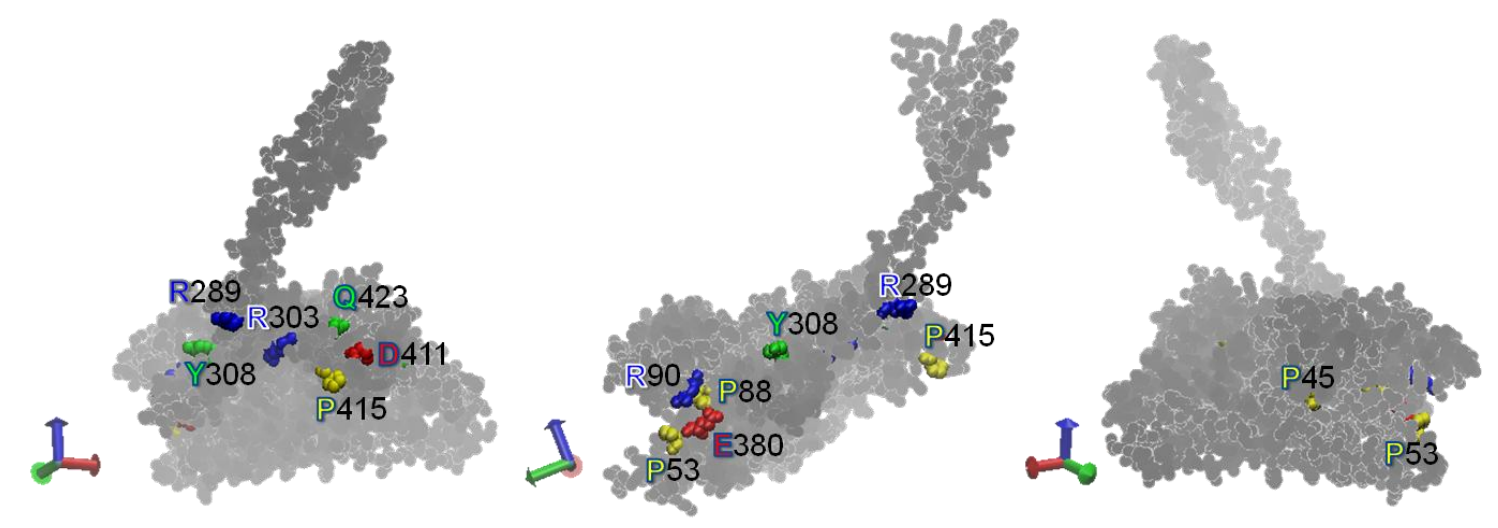

The BSL-1436 MCP model shows the spatially conserved amino acids in 17 taxa. The single model is rotated in three separate orientations to feature the perimeters or edges of the protein that are involved in subunit binding interactions. Conserved amino acids are shown in "VDW" mode and colored by "Residue Type"; polar = green, non-polar = yellow, acidic $=$ red and basic $=$ blue. The rest of the model is rendered in "Points" mode with the non-conserved residues shown as gray shaded spheres. Darker shading in "Depth Cueing" mode indicates atoms that are nearer to the viewer or projecting out of the page, and lightly shaded atoms are projecting into the page, away from the viewer. Conserved amino acids that remain exposed near the surface of the protein are labeled and numbered according to the BSL-1436 sequence position. 
Figure 2.8 Spatial patterns of amino acid sequence conservation in the BSL-1436

\section{MCP assembly}

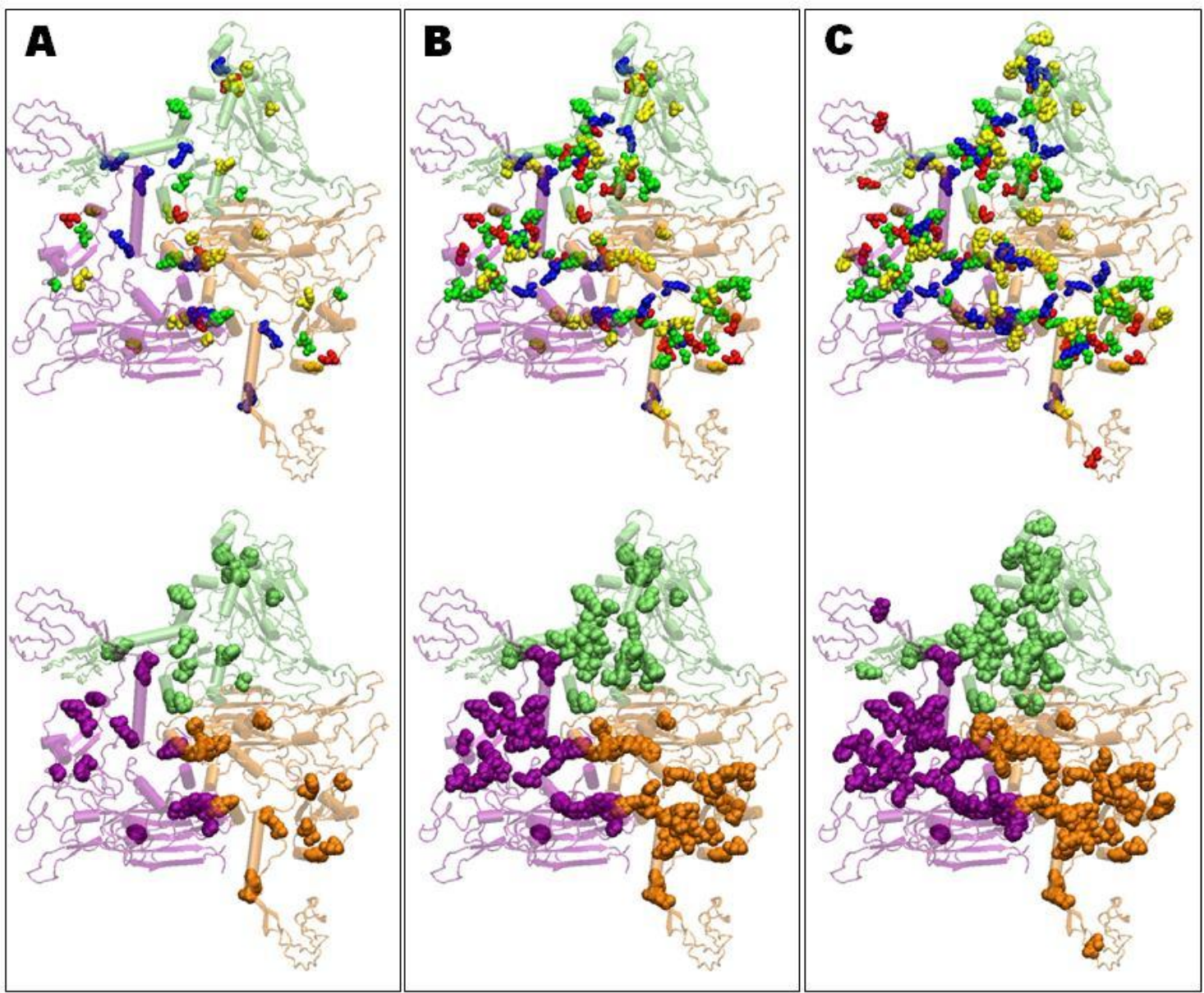

Panels A, B and C show three homology models of BSL-1436 MCPs in their predicted configuration within an assembled virion, interacting at 2-, 3-, and 5-fold symmetry axes. Each MCP subunit is rendered in "Cartoon" mode, showing $\alpha$-helices as cylinders and $\beta$ strands as arrows, and colored separately to distinguish it from the others. In panels $\mathrm{A}, \mathrm{B}$, and C, homology models of BSL-1436 depict clade-specific identical residues superimposed on the "Cartoon" rendered structures as spheres. Images on the top show spatially identical amino acids in "VDW" mode and colored by "Residue Type"; polar = green, non-polar $=$ yellow, acidic $=$ red and basic $=$ blue. Images on the bottom show amino acids rendered in "VDW" mode and colored by subunit association. Panel A) shows the identical residues conserved among Gokusho- (11 taxa), Picho- (5 taxa), and Alpavirinae (1 taxon) MCPs. Panel B) shows identical residues within the Gokusho- (11) and Picho- (5) clades, and panel C) depicts the identical amino acid residues in the Gokusho- (11) group only. 
Figure 2.9 PQR solvent accessibility mapping of capsid proteins

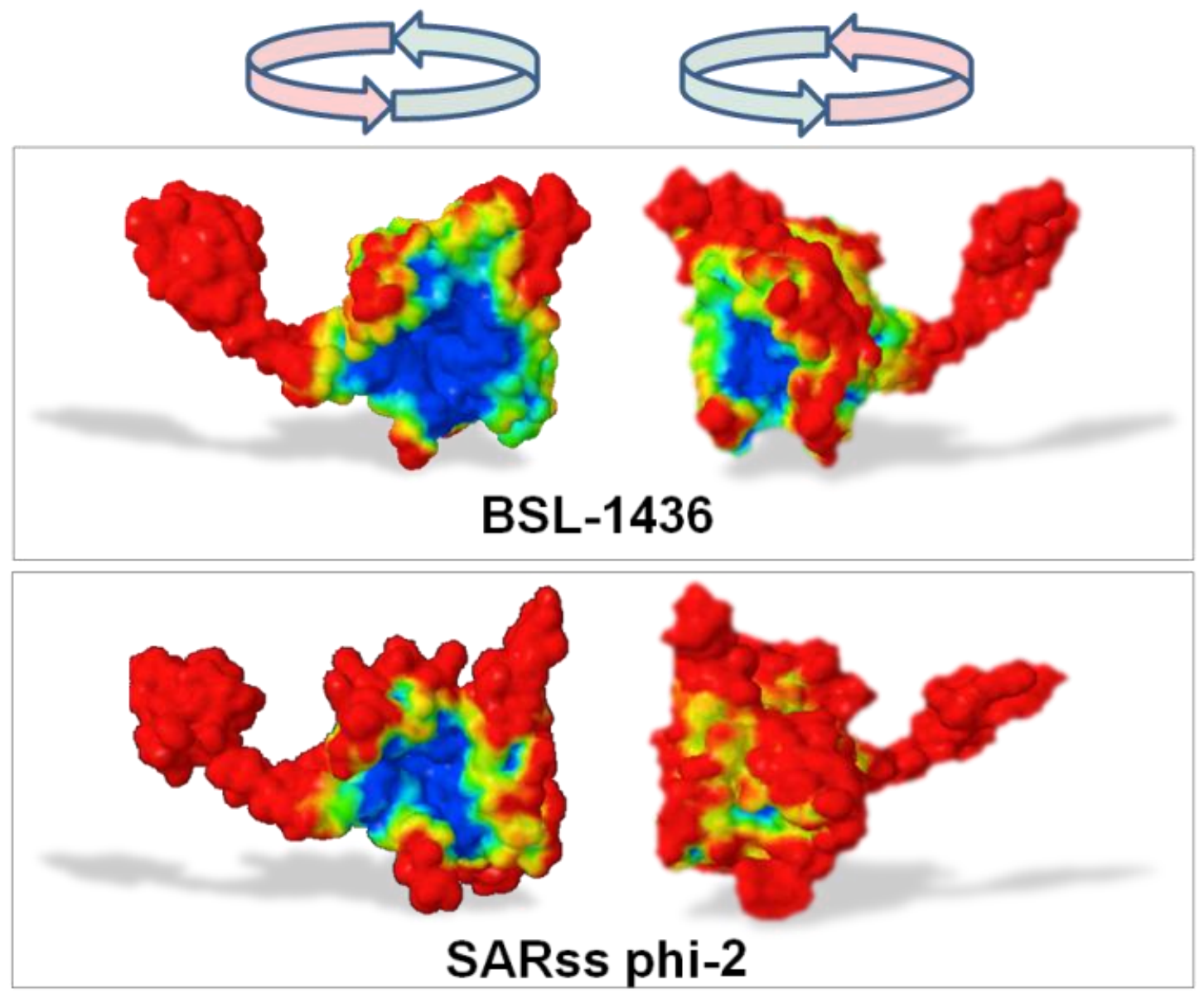

Solvent accessibility models based on Poisson-Boltzmann electrostatic calculations are mapped to the surface of the major capsid protein. Heat-map coloration spans the spectrum from red to blue: Red indicates maximum kT/e regions of the protein that are highly accessible to solvent and blue indicates minimum kT/e, or regions less accessible to solvent. The models shown represent the two proteins with the highest (BSL-1436) and lowest (SARss phi-2) isoelectric points (Table 2.2). Each model is shown horizontally rotated approximately 180 degrees relative to the viewer. Models on the left show the surface of the protein facing the interior of the virion, and models shown rotated on the right highlight the saddle-shaped binding pocket associated with the 5-fold axis interaction. 


\section{CHAPTER 2 TABLES:}

Table 2.1 BLAST comparisons to the Spiroplasma phage-4 (SpV-4) major capsid protein reference sequence NP_598320.1

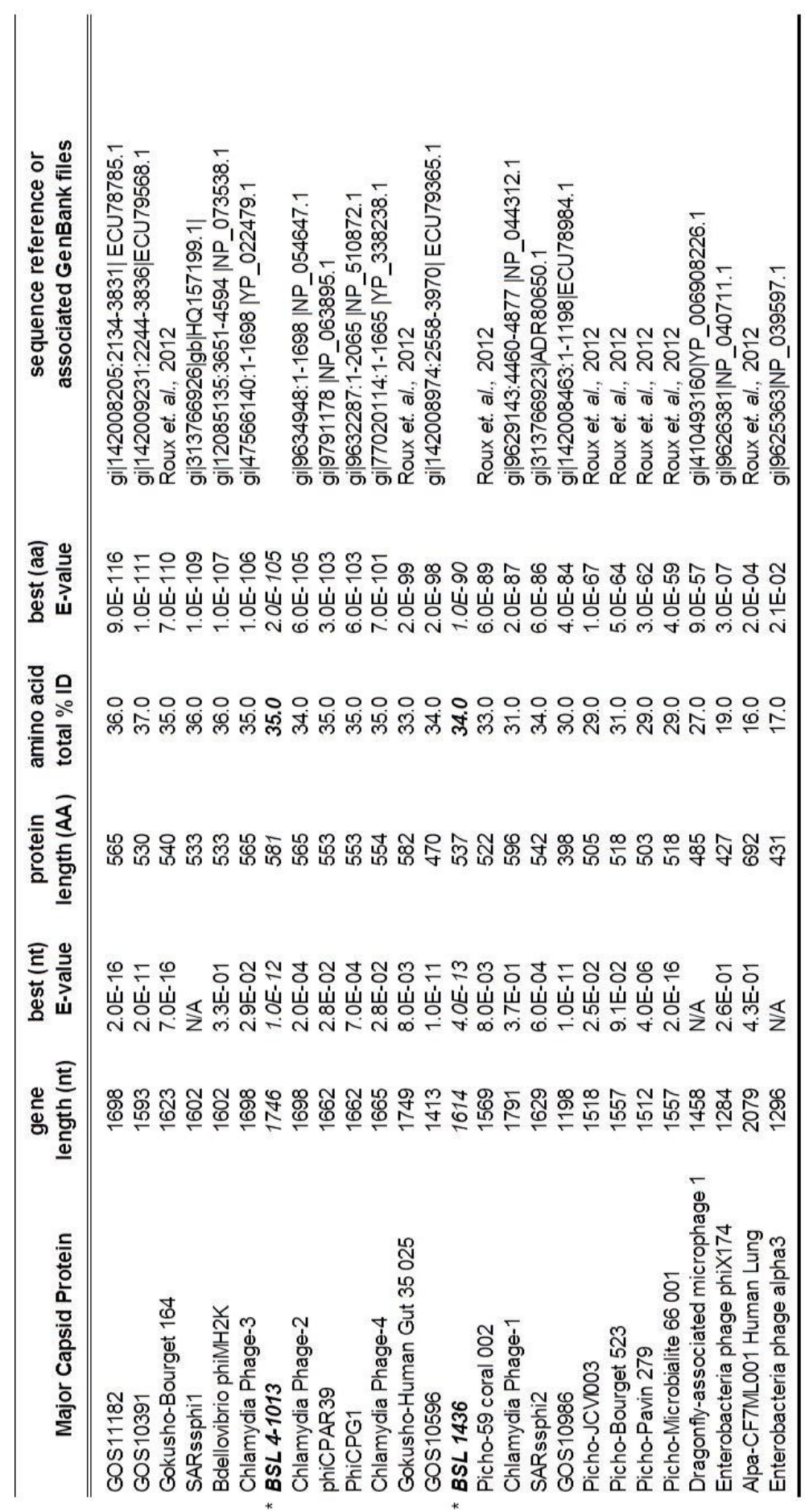


Table 2.1 description:

Twenty-four sequences, encompassing the Gokushovirinae, Pichovirinae and Alpavirinae microvirus subgroups from a wide range of physiochemical environments are shown in addition to the two BSL contigs 1436 and 1013 (Boldface). All sequences in the table are compared to the Spiroplasma phage-4 (SpV-4) major capsid protein gene (M17988.1, GI:334998, NP_598320.1). Needleman-Wunsch Global alignment [100] is used to estimate percent amino acid sequence identity values. BLAST2seq tBLASTn was used to produce E-values for nucleotide sequences, and BLASTp was used to produce E-values for amino acid sequences. "N/A" in the E-value column indicates that no significant similarity was found by BLAST alignment. SpV-4 was chosen as the reference sequence because its structure has been solved by cryo-electron microscopy and its Protein Data Bank structure (ID\#: 1KVP) is used for all structure-based analyses herein. 
Table 2.2 Capsid protein physiochemical properties as computed by the ExPASy ProtParam tool

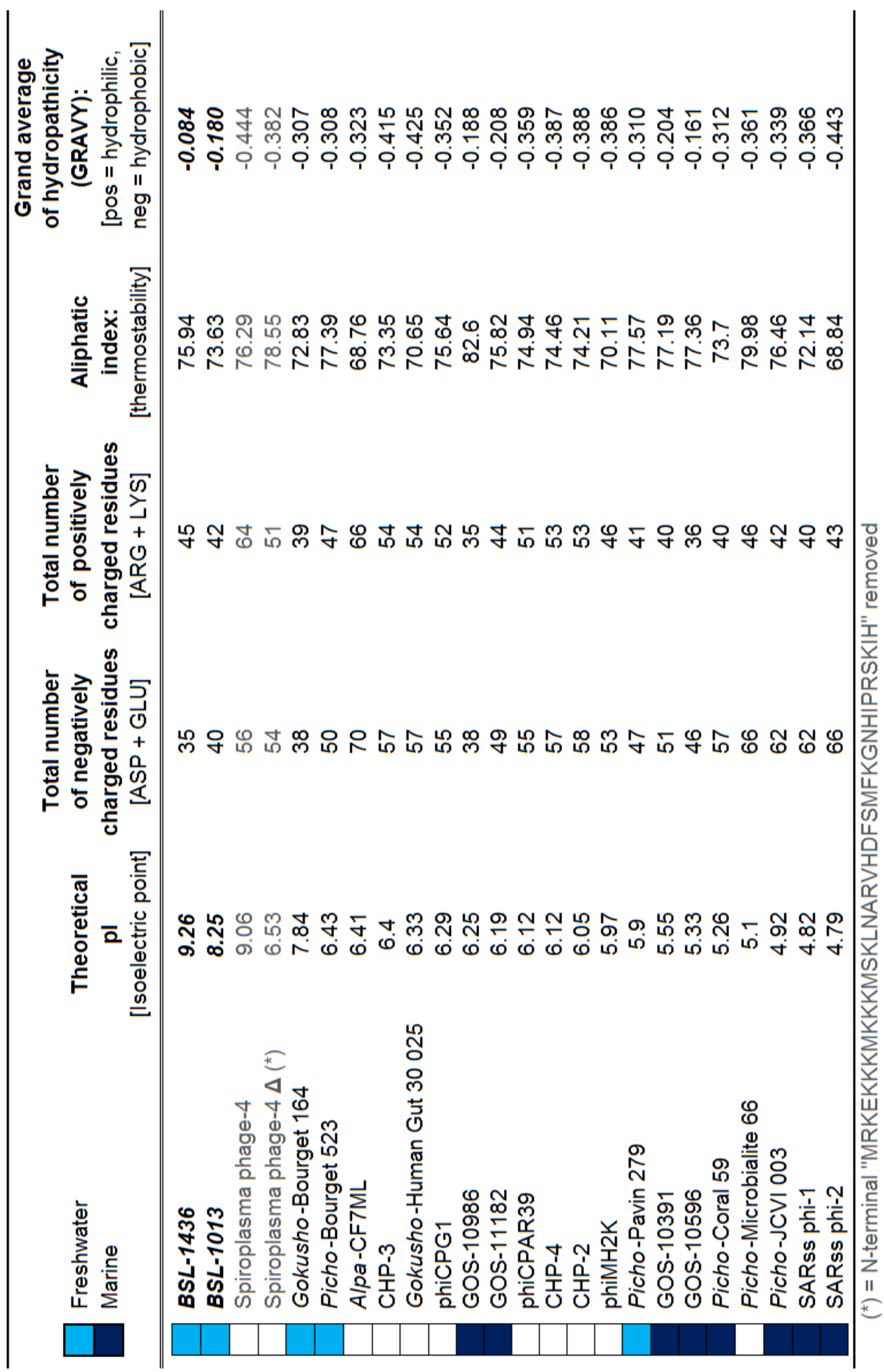


Table 2.2 description:

Twenty-one MCP sequences, encompassing the Gokushovirinae, Pichovirinae and Alpavirinae microvirus subgroups, from a wide range of physiochemical environments are shown in addition to the two BSL strains 1436 and 1013. Light blue squares indicate a freshwater habitat origin, and navy blue squares indicate marine habitats. The physiochemical property data is sorted by theoretical net isoelectric point (pI). BSL MCP sequences are shown in boldface type. Light gray type indicates $\mathrm{SpV}-4$ model values that have not been sorted by $\mathrm{pI}$. Thirty-seven N-terminal residues of the Spiroplasma phage- 4 GenBank sequence, which are not part of the cryo-EM structure 1KVP, have been removed to produce the Spiroplasma phage- $4 \Delta$ sequence. 


\title{
CHAPTER 2 SUPPLEMENTAL FIGURES:
}

\section{Supplemental Figure S-2.1 PROMALS3D colored structure-based alignment of 17 microvirus capsid protein sequences, based on $\mathrm{SpV}-4$ 1KVP structure file}

\author{
Major capsid protein sequences from the 17 microviruses for which homology models \\ were generated are shown in a structure-guided alignment using PROMALS3D. The \\ structure of the SpV-4 MCP was used to guide the alignment. The first line indicates \\ aligned positions with a conservation index above 5. The first and last residue numbers of \\ each sequence in each alignment block are shown on either side of the alignment. The \\ last two lines show the consensus amino acid sequence (CONSENSUS AA) and \\ consensus predicted secondary structure (CONSENSUS SS). Predicted alpha-helices are \\ indicated with an (h) highlighted red, and beta-strands with a blue-highlighted (e). \\ Identical consensus amino acid symbols are shown as bold, uppercase letters. \\ Additionally, conservation of amino acid types are labeled accordingly: aliphatic (I, V, \\ L): $\boldsymbol{l}$; aromatic (Y, H, W, F): @; hydrophobic (W, F, Y, M, L, I, V, A, C, T, H): $\boldsymbol{h}$; \\ alcohol (S, T): $\boldsymbol{o}$; polar residues (D, E, H, K, N, Q, R, S, T): $\boldsymbol{p}$; tiny (A, G, C, S): $\boldsymbol{t}$; small \\ (A, G, C, S, V, N, D, T, P): $\boldsymbol{s}$; bulky residues (E, F, I, K, L, M, Q, R, W, Y): $\boldsymbol{b}$; positively \\ charged $(\mathrm{K}, \mathrm{R}, \mathrm{H})$ : +; negatively charged $(\mathrm{D}, \mathrm{E})$ : -; charged (D, E, K, R, H): $\boldsymbol{c}$.
}

(Figure is shown on the following 5 pages) 


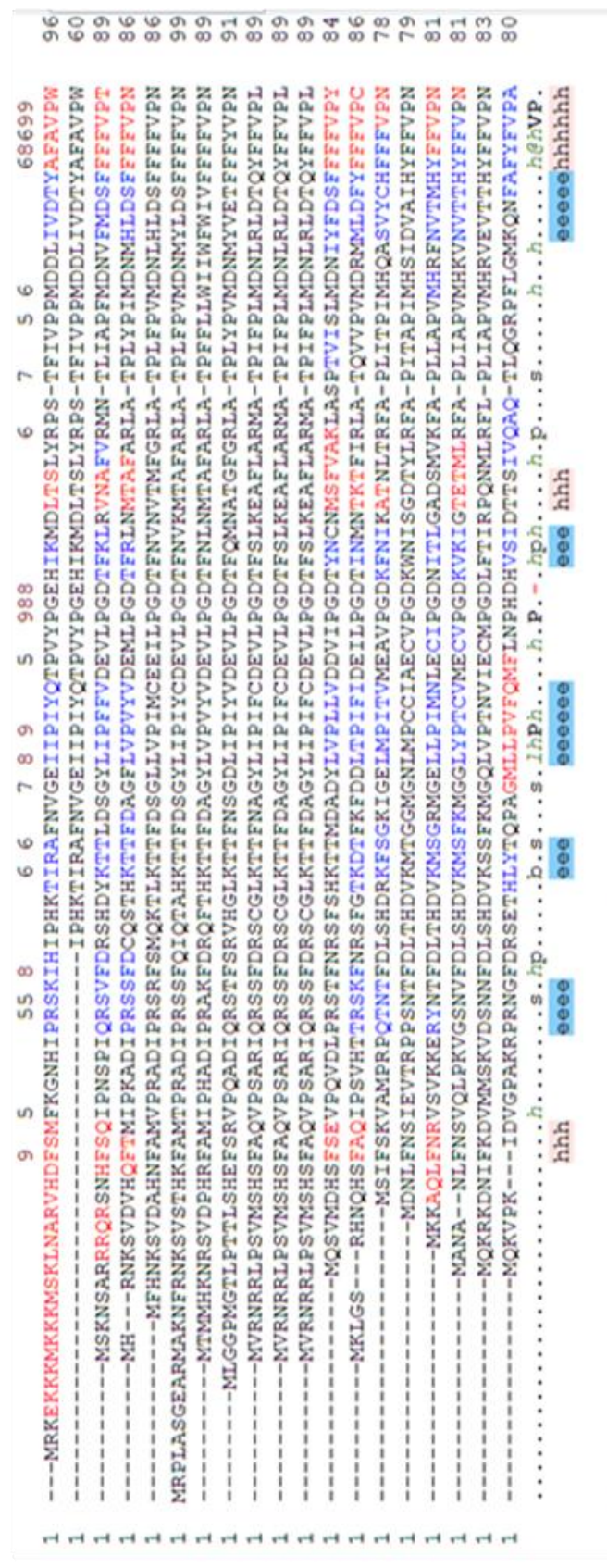

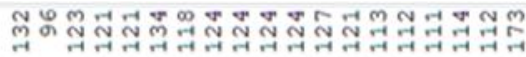

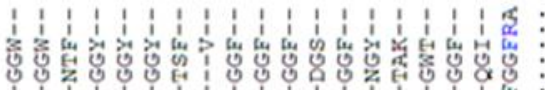

(1)

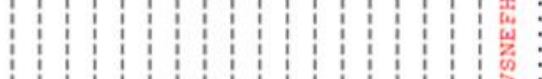

作

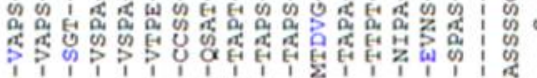

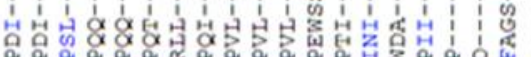

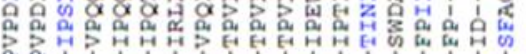

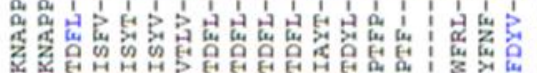

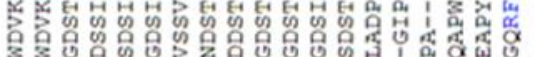

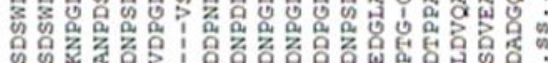

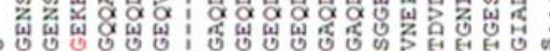

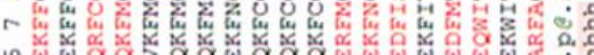

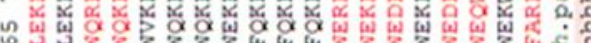

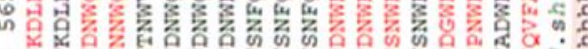

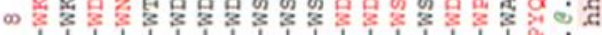

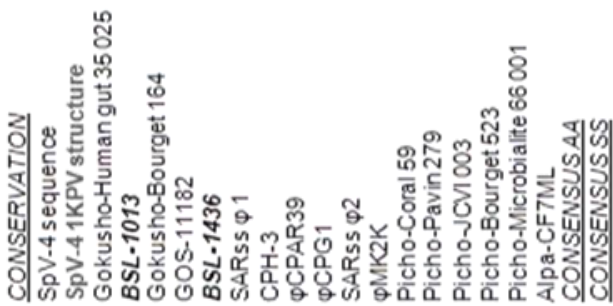

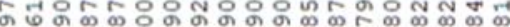

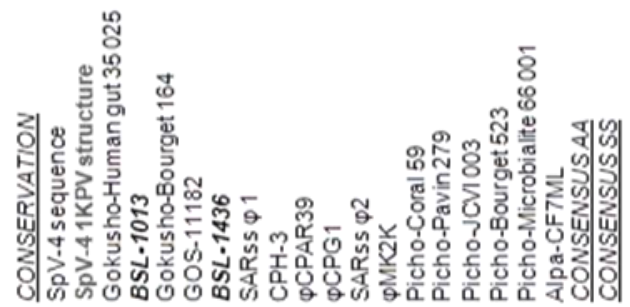

\section{1 of 5}




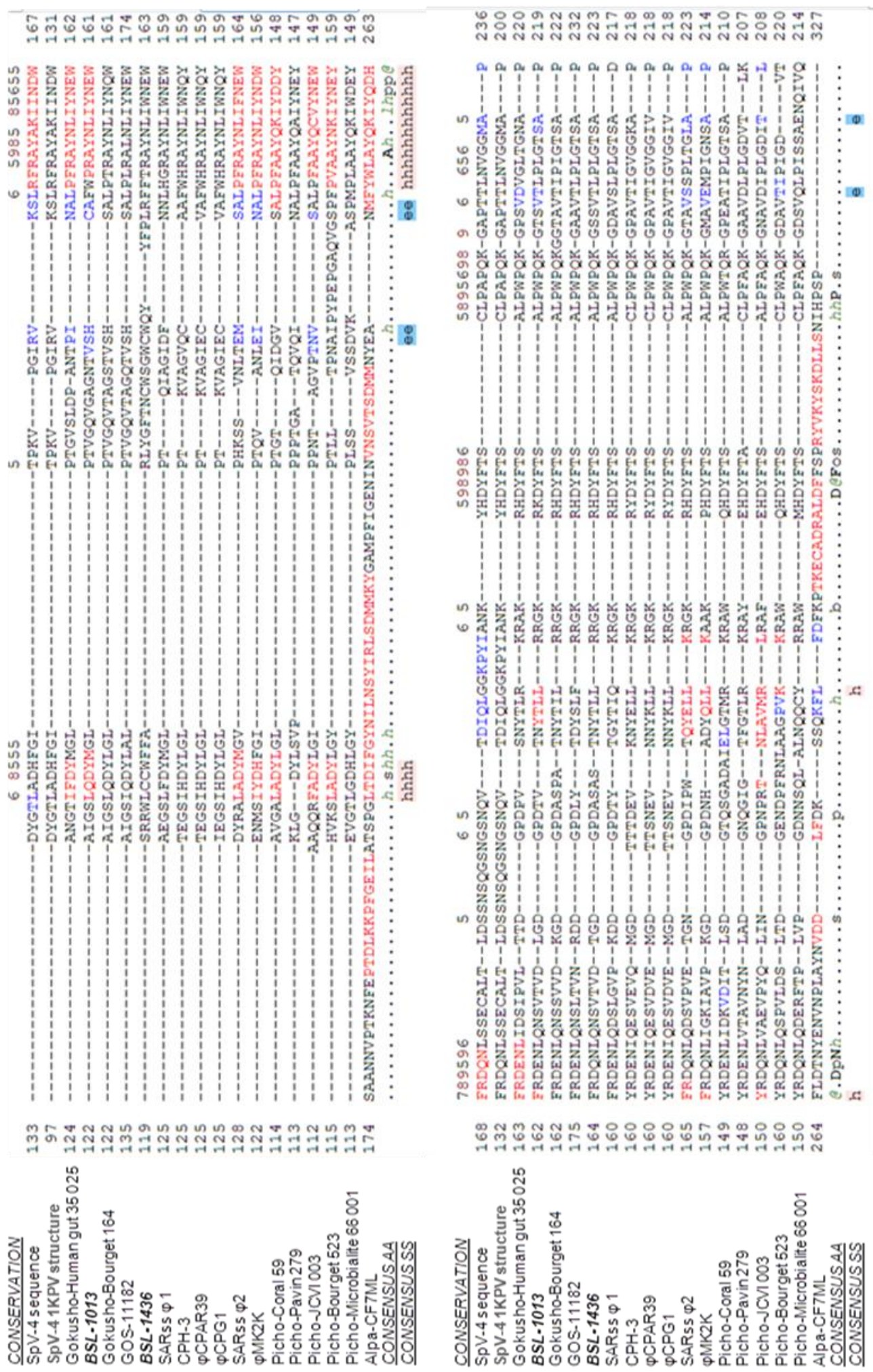

2 of 5 


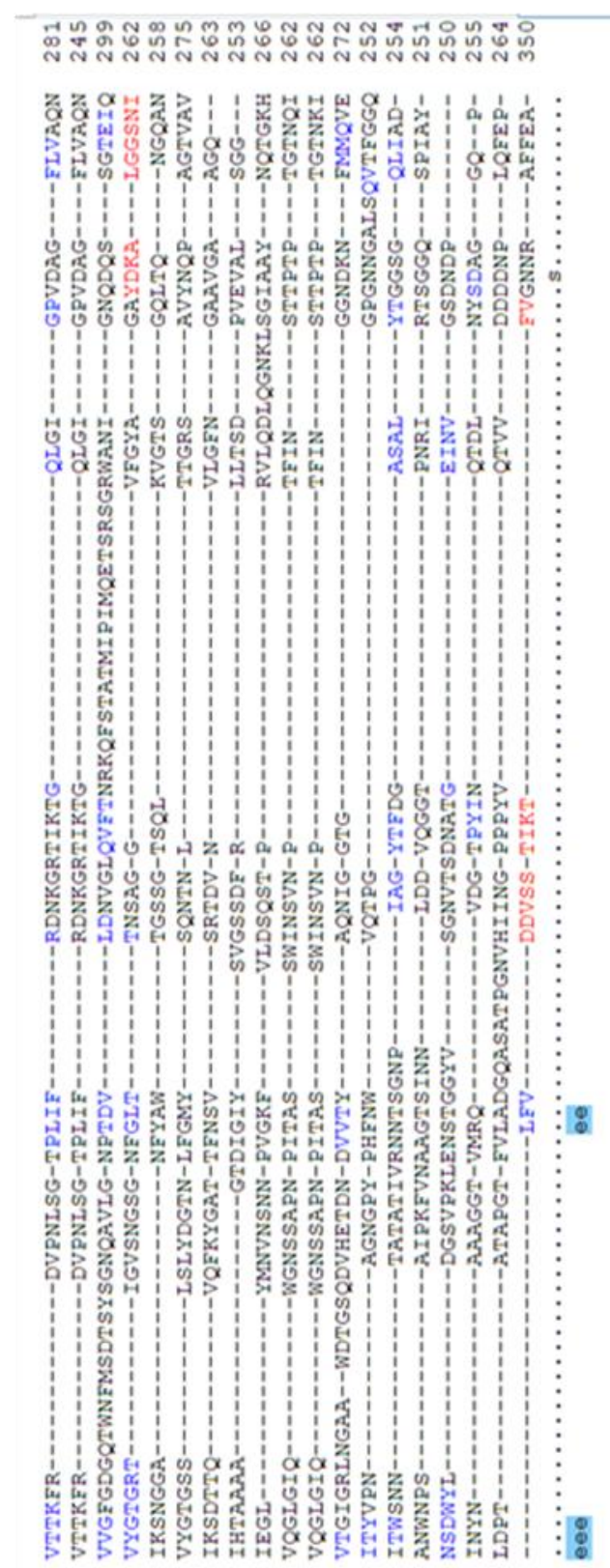

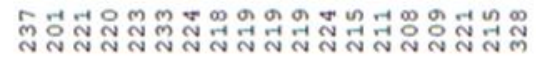

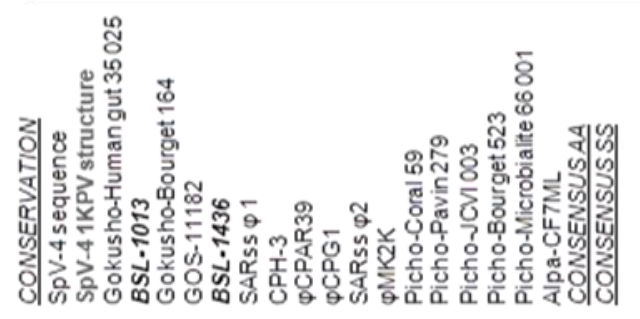

\section{3 of 5}

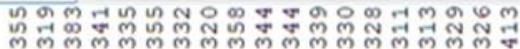

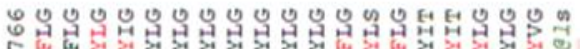
o

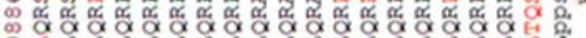

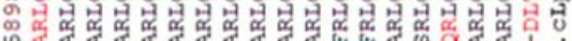

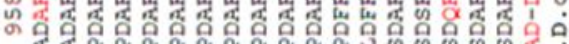
-

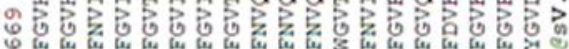

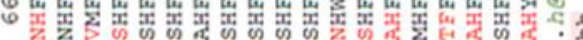

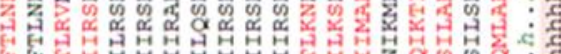

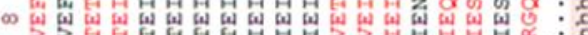

o 5 . ס

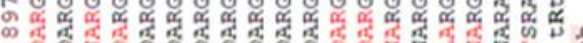

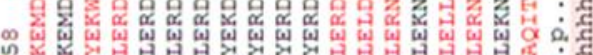

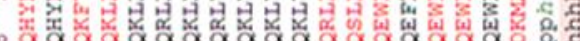

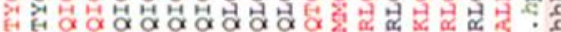

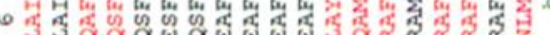

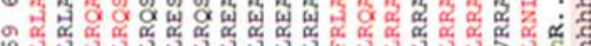

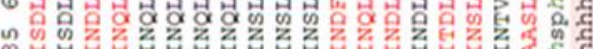

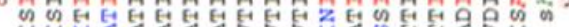

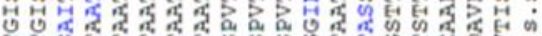

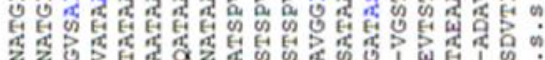
2

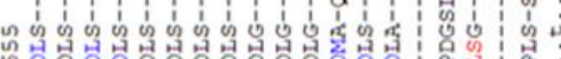

更

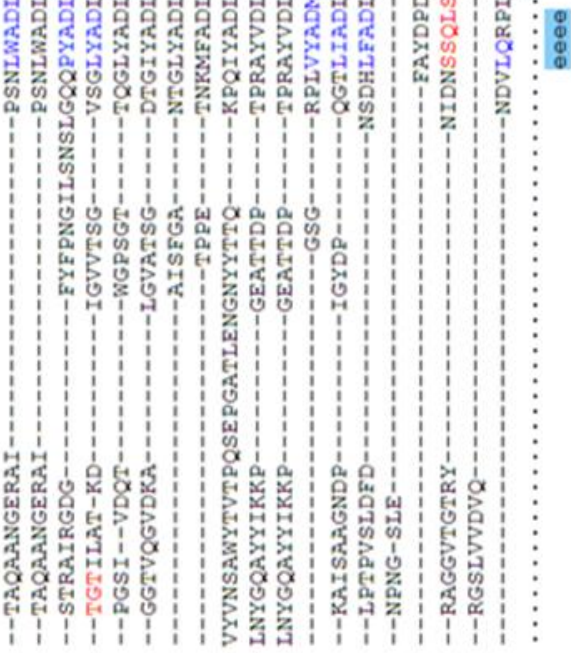

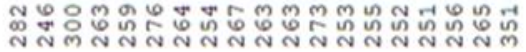

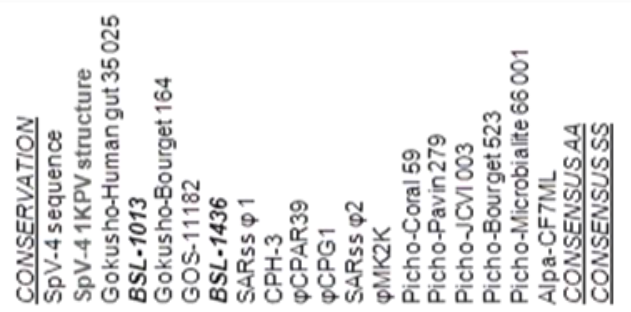




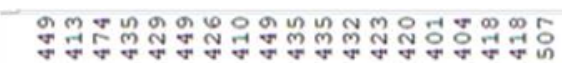

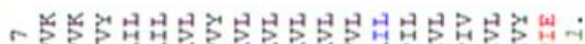

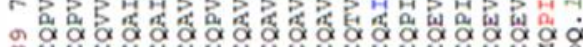

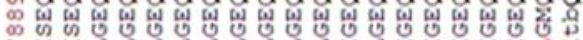

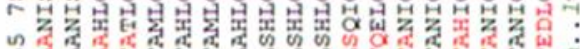

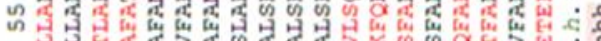

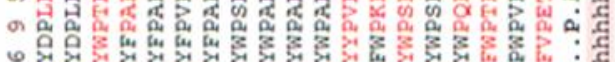

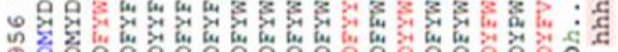

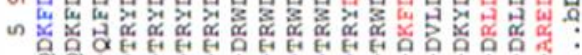

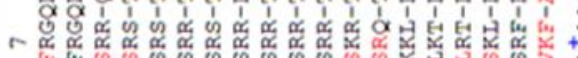

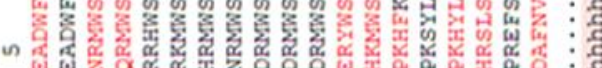

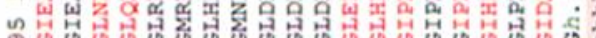

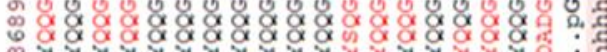

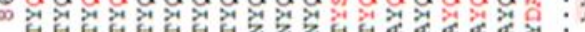

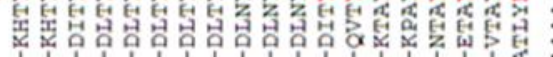

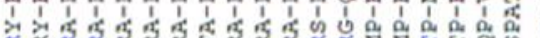

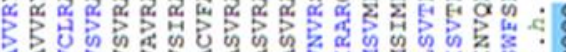

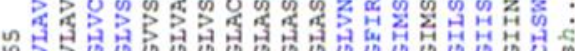

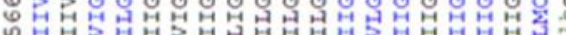

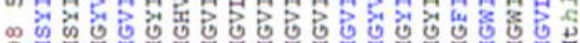

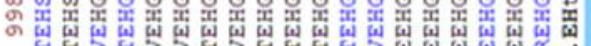

1

出

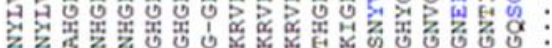

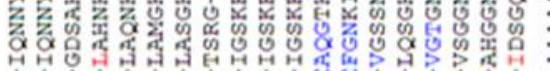

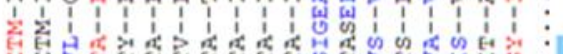

呅

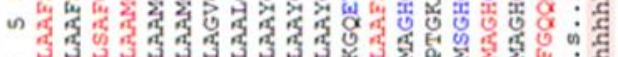

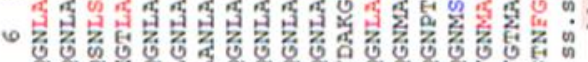

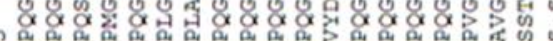

톨

这

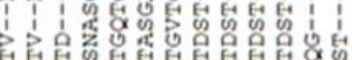

on

及与

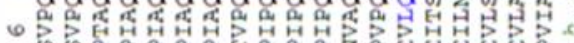

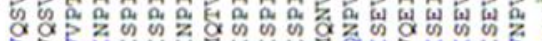

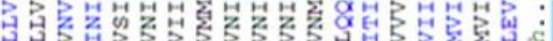

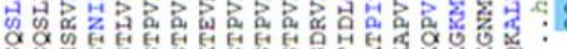

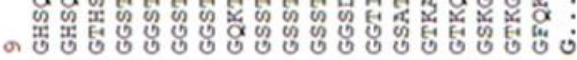

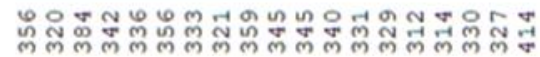

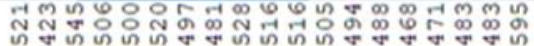

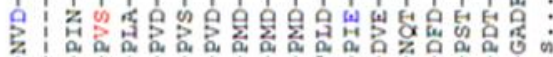

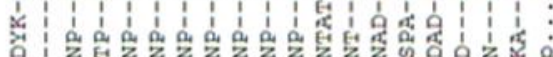

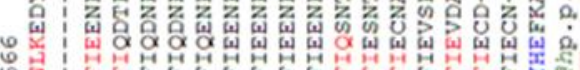

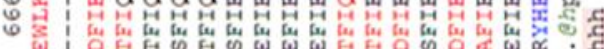

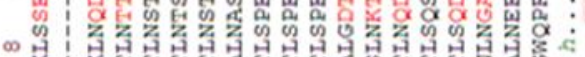

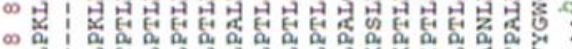

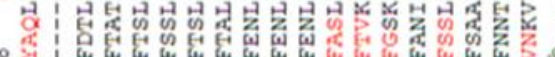

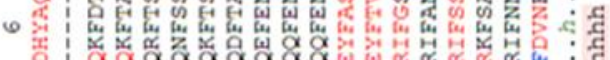

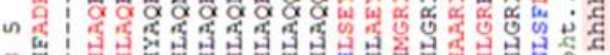

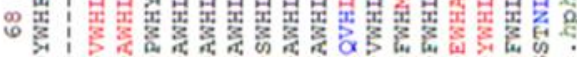

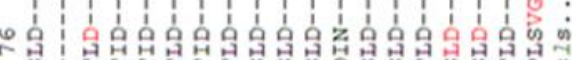

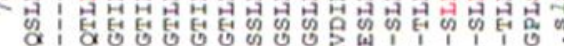

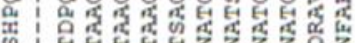

\%

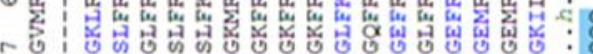

年

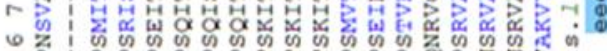

的峦

n

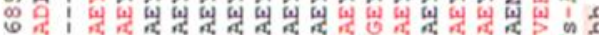

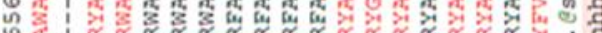

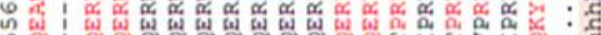

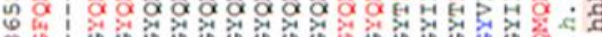

o

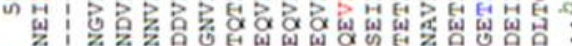

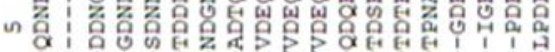

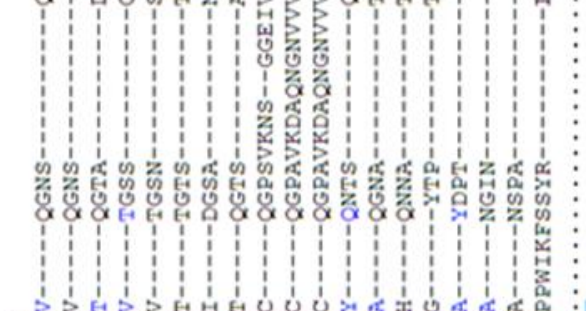

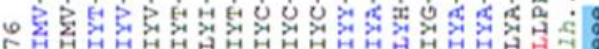

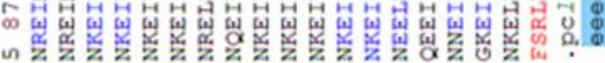

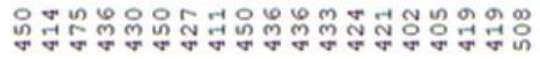

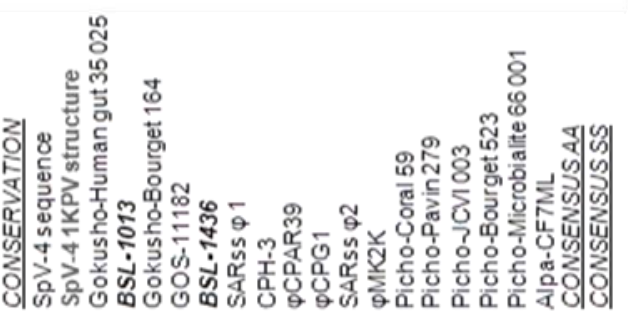

\section{4 of 5}




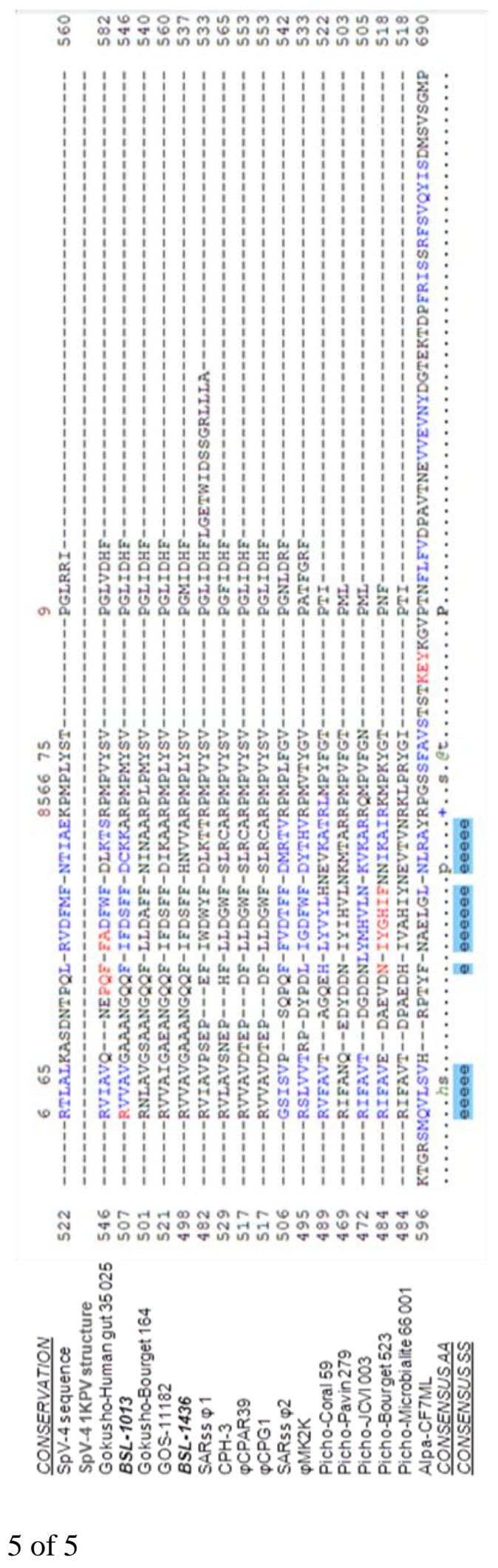




\section{CHAPTER 3:}

\section{A novel virus genome discovered in an extreme environment suggests recombination between unrelated groups of RNA and DNA viruses}

\section{CHAPTER 3 ABSTRACT}

Viruses are known to be the most abundant organisms on earth, yet little is known about their collective origin and evolutionary history. With exceptionally high rates of genetic mutation and mosaicism, it is not currently possible to resolve deep evolutionary histories of the known major virus groups. Metagenomics offers a potential means of establishing a more comprehensive view of viral evolution as vast amounts of new sequence data becomes available for comparative analysis.

Bioinformatic analysis of viral metagenomic sequences derived from a hot, acidic lake revealed a circular, putatively single-stranded DNA virus encoding a major capsid protein similar to those found only in single-stranded RNA viruses. The presence and circular configuration of the complete virus genome was confirmed by inverse PCR amplification from native DNA extracted from lake sediment. The virus genome appears to be the result of an RNA-DNA recombination event between two ostensibly unrelated virus groups. Environmental sequence databases were examined for homologous genes arranged in similar configurations and three similar putative virus genomes from marine environments were identified. This result indicates the existence of a widespread but previously undetected group of viruses. 
This unique viral genome carries implications for theories of virus emergence and evolution, as no mechanism for interviral RNA-DNA recombination has yet been identified, and only scant evidence exists that genetic exchange occurs between such distinct virus lineages.

\section{INTRODUCTION}

While viruses are known to be the most abundant organisms on earth, their collective evolutionary history, biodiversity and functional capacity is poorly understood $[58,112,113]$. Despite many inherent obstacles, viral metagenomics is enabling a more detailed evaluation of environmental viral diversity, and is burgeoning as an important tool for studying virus evolution $[64,114-116]$. Perhaps the greatest impediment to the evolutionary study of viruses is that there is no single phylogenetic marker in common among all viruses. Instead, "virus hallmark genes" [16] that are present in sub-groups of viruses are often used as the basis for taxonomic classification and evolutionary studies. However, lateral gene transfer between viruses complicates these analyses and virus classification schemes are likewise intensely debated [117-120].

The known virosphere consists of three principal viral types; the RNA-only viruses, which do not require a DNA intermediate in the replication cycle, viruses with DNA-based genomes, and retroid viruses that require the reverse transcription of their RNA into DNA during the virus life-cycle [120]. Lateral exchange of viral genes, via multiple possible mechanisms, is rampant among viruses within each of these principal 
types, but is generally confined to closely related viruses, or viruses (and plasmids) with similar replication mechanisms [121-128]. Clear examples of recent lateral gene transfer (LGT) from RNA-only to DNA-only viral types have not been observed.

We report the discovery of a group of circovirus-like DNA genomes whose common ancestor appears to have incorporated a capsid protein (CP) gene known previously only in RNA viruses. The mechanism responsible for the integration of the RNA virus cistron into the DNA virus, and the point in evolutionary time at which it occurred, are unclear. Relatively low levels of sequence divergence between the homologous viral proteins indicate the recombination event probably took place relatively recently. Moreover, it suggests that entirely new virus types may emerge via the lateral exchange of functional and structural modules from viruses of vastly different types, utilizing as yet unknown mechanisms.

\section{RESULTS AND DISCUSSION}

Analysis overview:

A metagenomics approach was used to investigate virus diversity in Boiling Springs Lake (BSL) located in Lassen Volcanic National Park, USA. BSL is an acidic, high temperature lake (ranging between $52^{\circ} \mathrm{C}$ and $95^{\circ} \mathrm{C}$, with a $\mathrm{pH}$ of approximately 2.2), which sustains a purely microbial ecosystem comprised of novel Archaea, Bacteria and several species of unicellular Eukarya [3, 4]. 
Initial analysis of the individual metagenomic DNA sequences from virus-sized particles obtained from BSL indicated the presence of a virus capsid protein $(\mathrm{CP})$ gene related to the downy mildew-infecting Sclerophthora macrospora-A (SmV-A) and Plasmopara halstedii-A (PhV-A) viruses $[129,130]$. SmV-A and PhV-A are unclassified linear, multipartite ssRNA viruses encoding capsid proteins similar to the plant-infecting Tombusviridae [130]. The BSL metagenomic sequences were then assembled into contigs and, surprisingly, a putative rolling circle replicase protein (Rep) gene most closely related to the circular ssDNA Circoviridae Rep was located immediately upstream of the ssRNA virus-like CP gene.

The complete circular genome was subsequently amplified from a native BSL DNA sample by inverse PCR using primers within the $\mathrm{CP}$ open reading frame (ORF). A native BSL DNA template was chosen for inverse PCR that was not used for metagenomic sequencing and was not pre-amplified with $\varphi 29$ polymerase, in order to rule out the possibility of spurious chimerism during sample preparation or sequence assembly. Big Dye Terminator (BDT) sequencing of the cloned viral genome was performed to confirm the original metagenomic sequence, to verify circularity, and to allow ORF prediction.

Translations of the predicted open reading frames for $\mathrm{CP}$ and Rep were used as search query sequences to compile a set of related virus genes from publicly available sequence databases. Individual phylogenies of the putative $\mathrm{CP}$ and Rep proteins were established using a subset of the search output sequences. Putative tertiary protein 
structures for Rep and CP were predicted by threading the ORF translations to homologous proteins with solved crystallographic structures.

\section{Genomic configuration:}

Since the novel BSL virus genome harbors genes homologous to both ssRNA and ssDNA viruses, it will be provisionally referred to herein as an "RNA-DNA hybrid virus", abbreviated "RDHV". Although the BSL RDHV genome is circular, the size of the genome is roughly double that of typical circoviruses, and the ORFs are arranged in an uncommon orientation (Figure 3.1A). The BSL RDHV Rep contains an N-terminal rolling circle replicase endonuclease (RCRE) domain (PF02407) [131] and a C-terminal Superfamily-3 RNA helicase (S3H) domain (PF00910), both of which are found in circoviral Reps [132]. A highly conserved DNA stem-loop in the intergenic region upstream of Rep is found in both BSL RDHV and Porcine circoviruses [133] (Figure 3.1B). The CP gene, however, is similar to those of the small icosahedral monopartite (+)ssRNA tombusviruses (PF00729).

BLAST searches and phylogenetic analysis indicate that the BSL RDHV Rep is more closely related to circoviral Reps than to those of other viruses or replicative plasmids (Figures 3.2 and 3.3A). Amino acid sequence alignments indicate significant conservation in both RCRE and S3H Rep domains (Figure 3.4). The N-terminal RCRE domain contains well-conserved motifs I, II and III. The putative $\alpha 3$-helix contains the motif III (YxxK) active-site tyrosine [134-137]. The S3H domain also contains wellconserved Walker-A, Walker-B, B' and C motifs [138-141]. These analyses, combined 
with the circularity of the genome and the presence of a circovirus-like DNA stem loop preceding Rep, indicate that the BSL isolate is a circovirus-like entity [132] and imply that the packaged genome is comprised of ssDNA.

BLAST searches and phylogenetic analysis indicates that the BSL RDHV capsid protein groups with the CPs of ssRNA viruses with multipartite genomes (SmV-A and $\mathrm{PhV}-\mathrm{A}$ ) and the monopartite ssRNA Tombusviridae, to the exclusion of capsid proteins found in ssDNA circoviruses, and plant-infecting nanoviruses and geminiviruses that also encode Rep (Figures 3.3B and 3.5). The SmV-A and PhV-A viruses are taxonomically categorized as unclassified Noda-like viruses. This assessment is based primarily on the RNA-dependent RNA-polymerase (RdRp) sequence and not the CP [142], which was apparently acquired from a tombusvirus-like ancestor [130]. As Melon Necrotic Spot Virus and several other tombusviruses are transported to the plants they infect by fungal vectors, it is likely that the multipartite $\mathrm{SmV}-\mathrm{A}$ and $\mathrm{PhV}-\mathrm{A}$, which infect downy mildews of plants, incorporated an RNA transcript encoding a tombusvirus-like CP.

Assessing structural homology in the RDHV proteins:

Several Tombusviridae capsid protein structures have been solved, including the ssRNA Tomato Bushy Stunt (TBSV) and Melon Necrotic Spot (MNSV) tombusviruses $[143,144]$. These structures have characteristic shell $(\mathrm{S})$ and projecting $(\mathrm{P})$ domains that are linked by a short hinge [145]. The N-terminal region that precedes the S-domain is referred to as the RNA-interacting, or R domain. The R domain, which allows the $\mathrm{CP}$ to interact with the viral RNA in the interior of the virion, is generally found to be 
unstructured and to contain basic, nucleic acid-interacting residues [143]. The connecting arm region (a), between the $\mathrm{R}$ and $\mathrm{S}$ domains, forms a $\beta$-annulus structure that connects three CPs at the 3-fold axes of symmetry in the virion [146]. A ClustalW multiple sequence alignment of the BSL and related CPs demonstrates different levels of conservation in each of the $\mathrm{CP}$ domain regions (Figure 3.6). The greatest level of sequence conservation is found within the $\mathrm{S}$ domain of these proteins [147]. Many of the Tombusviridae also contain a calcium ion binding motif (DxDxxD) in the S-domain interaction region $[148,149]$ that is thought to aid in uncoating of the virus when it enters the low calcium environment of the plant cell cytoplasm [150]. Replacing aspartic acids D155 and D157 with asparagines $(\mathrm{N})$ in the motif created a calcium ion binding-deficient mutant of the Turnip Crinkle Virus (TCV) CP [150]. Remarkably, the very same amino acid substitutions are present in the BSL RDHV capsid protein (Figure 3.6).

Structural similarity was assessed for the $\mathrm{CP}$ and Rep proteins by threading the BSL RDHV sequences to homologous solved structures. To substantiate the hypothesis that the BSL RDHV CP conforms to the S-P domain configuration, the BSL RDHV CP structure was predicted by threading using the known structures of the MNSV (PDB ID: 2ZAH) and TBSV (PDB ID: 2TBV) CPs (Figure 3.7A). The Z-scores for the structure predictions were 71.1 and 54.4, respectively (a Z-score above 10 indicates a high reliability model). The predicted structure of the BSL RDHV capsid protein was highly congruent to the S-P domain architecture found in the ssRNA TBSV and MNSV tombusviruses. The $\mathrm{S}$ domain is a canonical $\beta$-barrel jelly-roll fold consisting of nine antiparallel $\beta$-strands and two $\alpha$-helices located between the $\beta-2 / \beta-3$, and $\beta-4$ / $\beta-5$ 
strands [151]. The P-domain is in a $\beta$-barrel configuration composed of 8 antiparallel $\beta$ strands, with an additional $\beta$-turn between the hinge and P-domain. No $\alpha$-helices are present in the P-domain. The BSL RDHV is the only known DNA viruses harboring the S-P domain architecture, which is otherwise found exclusively in ssRNA viruses.

Structures for the BSL Rep nuclease and S3H regions were also predicted with high confidence based on the circular ssDNA Porcine Circovirus-2 (PCV2) Rep nuclease domain (PDB ID: 2HW0, Z-score = 128) [136] (Figure 3.7B) and the papillomavirus E1 multimeric helicase domain (PDB ID: 1TUE, Z-score $=68$ )[152]. The predicted nuclease domain of the BSL RDHV Rep contains the active-site YxxK motif in the number-3 $\alpha$ helix similar to other viral Reps [153, 154] (Figure 3.7B). The predicted structure of the BSL RDHV Rep helicase domain is capable of being appropriately assembled in silico into a complete hexameric unit (data not shown).

Due to a lack of detectable sequence similarity, functions for BSL RDHV ORF-3 or ORF-4 cannot be proposed. ORF-3 and ORF-4 are not homologues of the tombusvirus ORFs with the same designation, as depicted in Figure 3.1A.

Identifying similar viruses in other environments:

To determine whether the BSL RDHV is endemic to Boiling Springs Lake, or whether it represents a larger group of viruses, environmental sequence databases were scanned for homologous CP and Rep sequences arranged in similar configurations. Sequences of both proteins were found to be similar to translated metagenomic DNA sequences derived from the Global Ocean Survey (GOS)[90]. The BSL RDHV Rep 
protein sequence was also similar to Entamoeba and Giardia integrated Rep-like sequences, possibly acquired from viruses or plasmids [155] (Figures 3.2, 3.3A and 3.4).

Three candidate BSL RDHV-like genomes were detected in marine environments. Although many GOS sequences are similar to either the BSL RDHV CP or Rep proteins, only two paired-end read scaffolds from the GOS contain both circovirus-like Rep and tombusvirus-like CP genes similar to the BSL RDHV (GOS 10665-10666 and GOS 6800-6801; GI:142008897 and GI:134313054, respectively) (Figures 3.2 through 3.6). The GOS $6800 \mathrm{CP}$ sequence is truncated and was thus not used in the multiple alignment or phylogenetic analysis, however the available sequence was sufficient for BLASTp comparison (Figures 3.3B, 3.5 and 3.6). The GOS 6801 Rep sequence (GI:142008897 / EBA57255.1), while similar to the BSL RDHV, also contains a putative parvoviral NS1 protein fold that has been identified in a number of marine metagenome circoviral Rep sequences [37], possibly indicating a history of lateral gene exchange between linear (parvoviral) and circular ssDNA virus groups. A shotgun sequence from the Sargasso Sea [156] (GI:129569619) was also identified, which contains contiguous $\mathrm{N}$ and C-terminal fragments of Rep and CP that are similar to the BSL RDHV sequences and are in the same orientation (data not shown). These data strongly suggest that previously undetected BSL RDH-like viruses are widespread in the marine environment, and are likely to be found in other environments as well. 


\section{Summary}

A parsimonious scenario explaining the provenance of the BSL RDHV and its relatives is that a DNA circovirus-like progenitor acquired a capsid protein gene from a ssRNA virus via reverse transcription and recombination. While the mechanisms responsible for interviral RNA-RNA and DNA-DNA recombination are well characterized [157-161], no mechanism has yet been proposed to account for the inferred instances of interviral RNA-DNA recombination [162-164]. Any instance in which an RNA cistron is converted into DNA and then integrated into a DNA genome presumably involves a reverse-transcriptase (RT) mechanism. However, no traces of an RT module exists in the BSL RDHV. The presence of non-retroviral RNA virus genes in cellular genomes [15, 165-169] suggests that some cellular mechanism exists that allows RNADNA recombination in lieu of a virus-derived RT. Although the group II intron retrohoming phenomenon [170] and transposon mediated exchanges have not been observed to mediate interviral lateral gene transfer, these or similar host cell-based mechanisms may have facilitated the formation of the BSL RDH-like viruses. Moreover, the ancestral host must have also been permissive to both circovirus-like DNA viruses and plant (or fungal)-like ssRNA viruses for interviral RNA-DNA recombination to have occurred.

As more viral metagenomic data are generated and analyzed, additional evidence of recombination between RNA and DNA virus groups will likely be discovered. Such findings would highlight the intriguing possibility that novel virus groups can emerge via recombination between highly disparate virus types. However, a dearth of similar 
examples of interviral RNA-DNA recombination would otherwise suggest that such events are either rare or perhaps ancient.

Considering the possibility that the recombination mechanism by which DNA viruses acquire RNA cistrons is ancient, it would have broad implications for the early evolution of viruses. As RNA viruses are believed to evolutionarily precede the emergence of DNA viruses [16, 17], determining the mechanism responsible for direct recombination between RNA and DNA viruses may help address how genes from the "RNA World" were first incorporated into nascent DNA-based genomes during the putative "Virus World" era, and thus further implicate viruses in the RNA-World to DNA-World transition $[17,18,171,172]$. In any case, the discovery of the BSL RDHlike virus group extends the modular theory of virus evolution $[16,29,173,174]$ to encompass a much broader range of possibilities than previously thought.

\section{MATERIALS AND METHODS}

\section{Metagenomic sample preparation:}

See CHAPTER 1, Materials and Methods section, "Metagenomic DNA sample preparation".

Bioinformatic analysis:

Analysis by tBLASTx [73] of the $c a$. 380,000 metagenomic sequence reads using MG-RAST [32] indicated the presence of ssRNA virus sequences. Contigs were assembled using the meta-assembler workflow in CAMERA [28]. 
Repeated terminal sequences in the contig indicated a circular genome. To confirm circularity and to rule out artificial chimera formation either by $\varphi 29$ polymerase amplification $[175,176]$ or spurious assembly, inverse PCR was used to amplify the complete virus genome from the BSL DNA sample that had neither been amplified with १29polymerase nor used for pyrosequencing. The reverse and forward primers (5'CCTATTGGTGAGCTGTGGGTTGA-3' and 5'-

GTATCGCGTAACTTTAAGGAAACCG-3') were used to amplify the complete circular genome. Extension from inverse PCR primers originates within the capsid protein ORF. The 4089 nucleotide virus genome was amplified with Phire polymerase (Finnzymes) $\left[98^{\circ} \mathrm{C}, 30 \mathrm{sec}\right.$. initial denaturation, followed by a touch-down stage of 8 cycles; $98^{\circ} \mathrm{C}, 5$ sec. denaturation, $72^{\circ} \mathrm{C}$ to $65^{\circ} \mathrm{C}, 5 \mathrm{sec}$. annealing decreasing $1^{\circ} \mathrm{C} / \mathrm{cycle}, 72^{\circ} \mathrm{C}$ extension for $1.5 \mathrm{~min}$. followed by 25 cycles of amplification; $98^{\circ} \mathrm{C}, 5 \mathrm{sec}$. denaturation, $65^{\circ} \mathrm{C}, 5$ sec. annealing, $72^{\circ} \mathrm{C}, 1.5 \mathrm{~min}$. extension, followed by a final $72^{\circ} \mathrm{C}$ extension for $3 \mathrm{~min}$.] The whole-genome PCR product was cloned into pCR-TOPO-Blunt (Invitrogen) using the manufacturer's instructions. Plasmid DNA was used for BDT sequencing. The BSL RDHV genome sequence has been submitted to GenBank: accession number JN900499. The highly conserved DNA stem loop (Figure 3.1B) in the BSL RDHV genome was detected using the Mfold v4.6 nucleic acid folding and hybridization web server by applying default settings at $70^{\circ} \mathrm{C}$ [177]. ORFs were predicted using Mold, Protozoan and Coelentrate codon tables, and related virus sequences were retrieved by two PSI-BLAST search iterations of NCBI nr/nt and env databases using default parameters (threshold $=$ 0.005). Unrelated Microviridae Rep sequences were manually chosen for comparison. 
The CP and Rep ORFs were compared against selected sequences using BLASTp to prepare BLASTp tables (Figures 3.2 and 3.5).

The 542 amino acid BSL RDHV capsid protein ORF sequence was aligned to closely related sequences retrieved from PSI-BLAST searches using ClustalW with default parameters in MEGA v.5 [101] (Pairwise alignment: gap opening penalty $=10$, gap extension penalty $=0.1$. Multiple alignment: gap opening penalty $=10$, gap extension penalty $=0.2$. Protein weight matrix $=$ Gonnet. Delay divergent cutoff $=30 \%$ ) and then refined by hand. The phylogenetic tree was inferred using the Neighbor-Joining method [102] by applying a bootstrap test with 1000 replicates [111]. The 418 amino acid replicase ORF sequence alignment and phylogenetic tree was prepared using the same parameters as in the $\mathrm{CP}$ alignment. Both $\mathrm{CP}$ and Rep ClustalW multiple sequence alignment figures were prepared using Jalview [178].

Each ORF was analyzed using InterProScan $[179,180]$ to locate conserved protein domains within each ORF. Tertiary protein structures were first predicted by threading using the CPH Model Server [104], which automatically selects an appropriate solved protein structure as a scaffold. Structure predictions were confirmed and refined using EsyPred3D [181], by entering Protein Data Bank (PDB) structure scaffolds manually. Structure predictions of BSL virus proteins were compared to solved crystallographic structures using the MultiSeq application [182] in VMD [106] (Figures 3.7A and 3.7B). 


\section{CHAPTER 3 FIGURES:}

Figure 3.1 Organiztion of the BSL RDHV genome

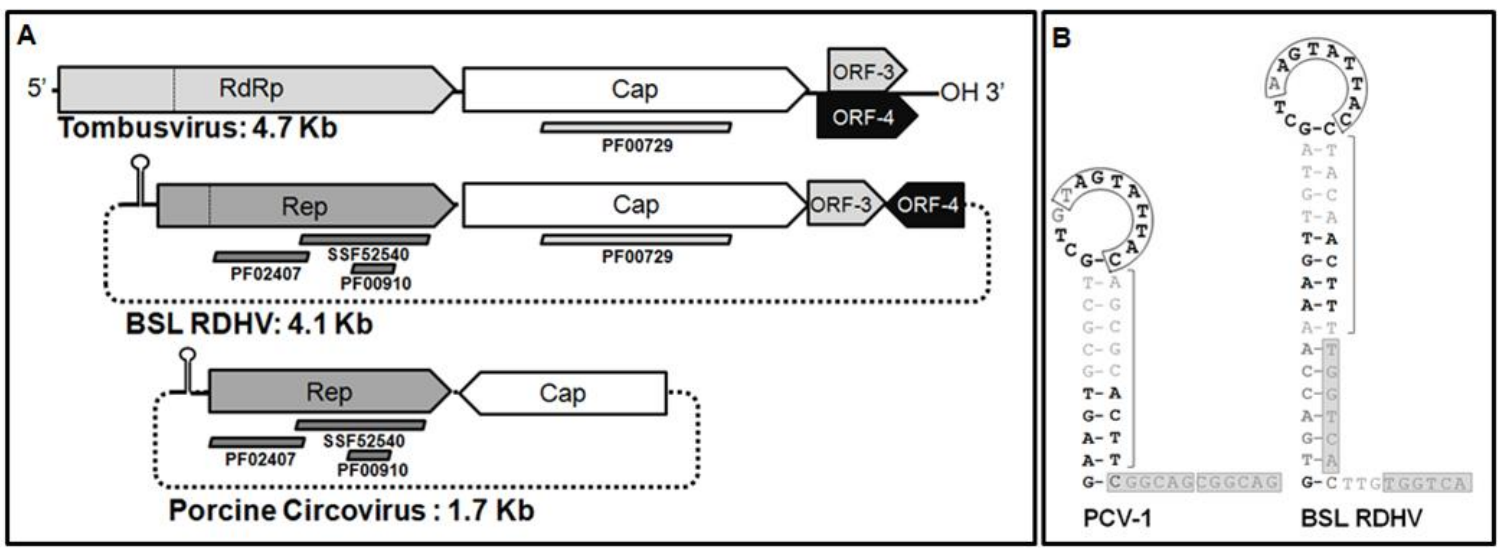

(A) Schematic representations of tombusvirus, BSL RDHV and Porcine circovirus (PCV) genomes: Tombusviruses are linear ssRNA viruses, BSL RDHV and PCV are circular ssDNA viruses. Bars below ORFs indicate protein families detected by InterProScan. ORFs 3 and 4 in BSL RDHV and Tombusvirus genomes are not homologous. (B) Comparison of PCV-1 and BSL RDHV stem-loops: Both BSL RDHV and circoviruses have a conserved stem loop in the intergenic region upstream of Rep. Black text indicates sequence identity. Nonanucleotide replication origins in the 13nt loop are boxed. Brackets indicate the 9 nucleotide stem preceding hexamer repeats $\mathrm{H} 1$ and $\mathrm{H} 2$ (gray boxes). 
Figure 3.2 BLASTp data for Rep amino acid sequences

\begin{tabular}{|c|c|c|c|c|c|c|}
\hline \multicolumn{7}{|c|}{ BLASTp RESULTS FOR REP PROTEINS } \\
\hline Accession & Description & Max score & Total score & Query coverage & Evalue & Max ident \\
\hline & Global Ocean Survey & & & & & \\
\hline 142008899 & GOS_10666 & 156 & 169 & $67 \%$ & $7.0 \mathrm{E}-49$ & $50 \%$ \\
\hline \multirow[t]{2}{*}{134313056} & GOS_6801 & 132 & 147 & $67 \%$ & $4.0 \mathrm{E}-40$ & $43 \%$ \\
\hline & Circovirus & & & & & \\
\hline 189916555 & PCV2 & 149 & 149 & $79 \%$ & $2.0 \mathrm{E}-45$ & $33 \%$ \\
\hline \multirow[t]{2}{*}{96980765} & StCV & 140 & 140 & $79 \%$ & $2.0 \mathrm{E}-42$ & $33 \%$ \\
\hline & Cyclovirus & & & & & \\
\hline \multirow[t]{2}{*}{324309805} & DfCyV & 114 & 147 & $61 \%$ & $3.0 \mathrm{E}-33$ & $38 \%$ \\
\hline & Integrated Protozoan & & & & & \\
\hline 253743285 & Giardia intestinalis ATCC 50581 & 115 & 149 & $76 \%$ & $6.0 \mathrm{E}-33$ & $35 \%$ \\
\hline \multirow[t]{2}{*}{183234913} & Entamoeba histolytica HM-1-IMSS & 56.2 & 70.8 & $40 \%$ & $4.0 \mathrm{E}-14$ & $32 \%$ \\
\hline & Phytoplasmal plasmids & & & & & \\
\hline 13434985 & Onion yellows phytoplasma & 46.2 & 46.2 & $29 \%$ & $7.0 \mathrm{E}-10$ & $29 \%$ \\
\hline \multirow[t]{2}{*}{327202124} & Periwinkle leaf yellowing phytoplasma & 43.1 & 59.3 & $33 \%$ & $6.0 \mathrm{E}-09$ & $44 \%$ \\
\hline & Nanovirus & & & & & \\
\hline 71532925 & BBTV & 40 & 72.4 & $37 \%$ & $4.0 \mathrm{E}-08$ & $57 \%$ \\
\hline \multirow[t]{2}{*}{20530225} & SCSV & 40 & 73.9 & $34 \%$ & $4.0 \mathrm{E}-08$ & $36 \%$ \\
\hline & Geminivirus & & & & & \\
\hline 20564197 & TPCTV & 21.9 & 84.3 & $37 \%$ & $2.4 \mathrm{E}-02$ & $57 \%$ \\
\hline \multirow[t]{2}{*}{10257478} & HrCTV & 13.9 & 13.9 & $5 \%$ & $7.8 \mathrm{E}+00$ & $33 \%$ \\
\hline & Microvirus & & & & & \\
\hline 9791179 & CPAR39 & 17.7 & 17.7 & $2 \%$ & $5.2 \mathrm{E}-01$ & $55 \%$ \\
\hline 12085145 & phiMH2K & 14.2 & 14.2 & $1 \%$ & $6.8 \mathrm{E}+00$ & $63 \%$ \\
\hline
\end{tabular}

The BSL RDHV Rep ORF amino acid sequence was compared to related Rep sequences using BLASTp. Output parameters are shown. Virus family designations are indicated when possible. (GOS) Global Ocean Survey paired-end read scaffolds 10666 and 6801. (PCV2) Porcine Circovirus-2, (StCV) Starling Circovirus, (DfCyV) Dragonfly Cyclovirus. (BBTV) Banana Bunchy Top Virus, (SCSV) Subterranean Clover Stunt Virus. (TPCTV) Tomato Pseudo Curly Top Virus, (HrCTV) Horseradish Curly Top Virus. 
Figure 3.3 Rolling circle replicase (Rep) and capsid protein (CP) phylogenies

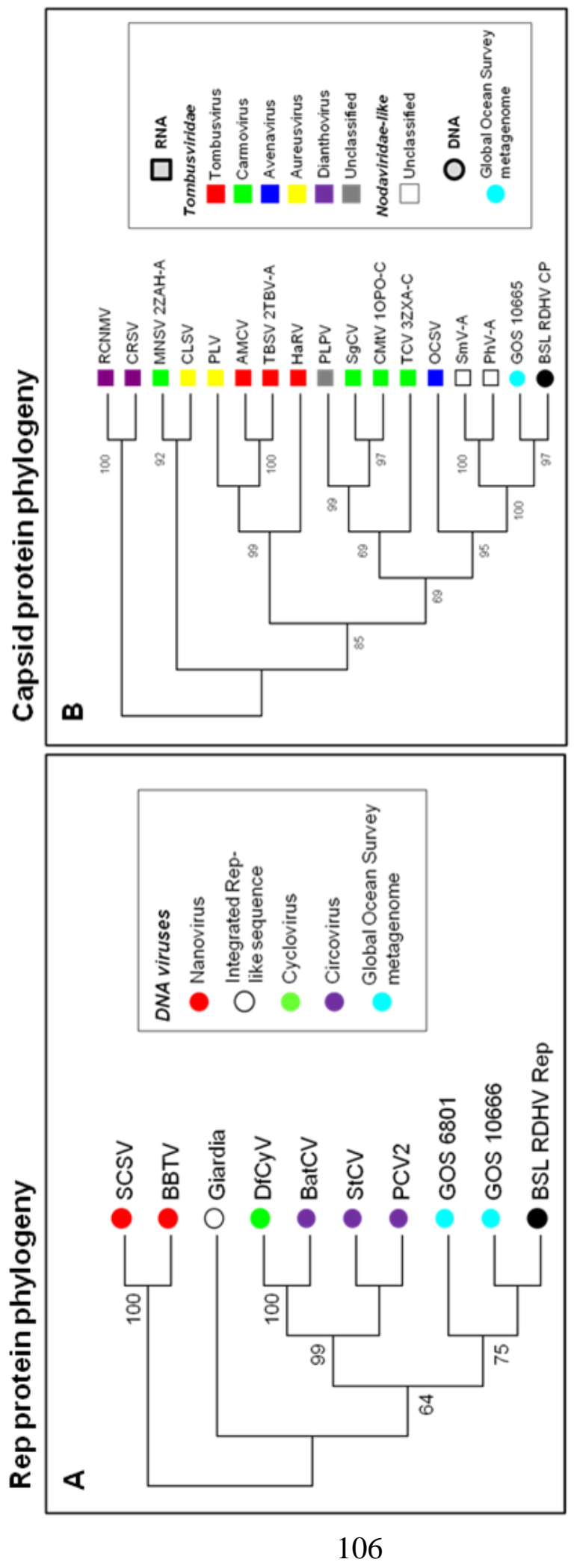


Figure 3.3 description:

(A) The Rep protein phylogeny was inferred from 204 conserved amino acid positions using the Neighbor-Joining method. Bootstrap values above a $60 \%$ significance threshold, based on 1000 replicates, are shown next to the branches.

NANOVIRIDAE: (SCSV) Subterranean Clover Stunt Virus, (BBTV) Banana Bunchy Top Virus. CIRCOVIRIDAE: (DfCyV) Dragonfly Cyclovirus, (BatCV) Bat Circovirus, (StCV) Starling Circovirus, (PCV2) Porcine Circovirus-2. (GOS) Global Ocean Survey paired-end read scaffolds 6801 and 10666. (B) The capsid protein (CP) phylogeny was inferred from 256 conserved amino acid positions using the Neighbor-Joining method. Bootstrap values above a $60 \%$ significance threshold, based on 1000 replicates, are shown next to the branches. TOMBUSVIRIDAE: Dianthoviruses; (RCNMV) Red Clover Necrotic Mosaic Virus, (CRSV) Carnation Ringspot Virus. Aureusviruses: (CLSV) Cucumber Leaf Spot Virus, (PLV) Pothos Latent Virus. Tombusviruses; (AMCV) Artichoke Mottled Crinkle Virus, (TBSV) Tomato Bushy Stunt Virus, (HaRV) Havel River Tombusvirus. Unclassified Tombusviridae; (PLPV) Pelargonium Line Pattern Virus. Carmoviruses; (MNSV) Melon Necrotic Spot Virus, (SgCV) Saguaro Cactus Virus, (CMtV) Carnation Mottle Virus, (TCV) Turnip Crinkle Virus. Avenavirus; (OCSV) Oat Chlorotic Stunt Virus. NODAVIRIDAE-like: (SmV-A) Sclerophthora macrospora Virus-A, (PhV-A) Plasmopara halstedii Virus-A. (GOS) Global Ocean Survey paired-end read sequence 10665 . Virus proteins for which structures are available are listed with the Protein Data Bank (PDB) identification code after the virus abbreviation. 
Figure 3.4 Rolling circle replicase (Rep) amino acid multiple sequence alignment

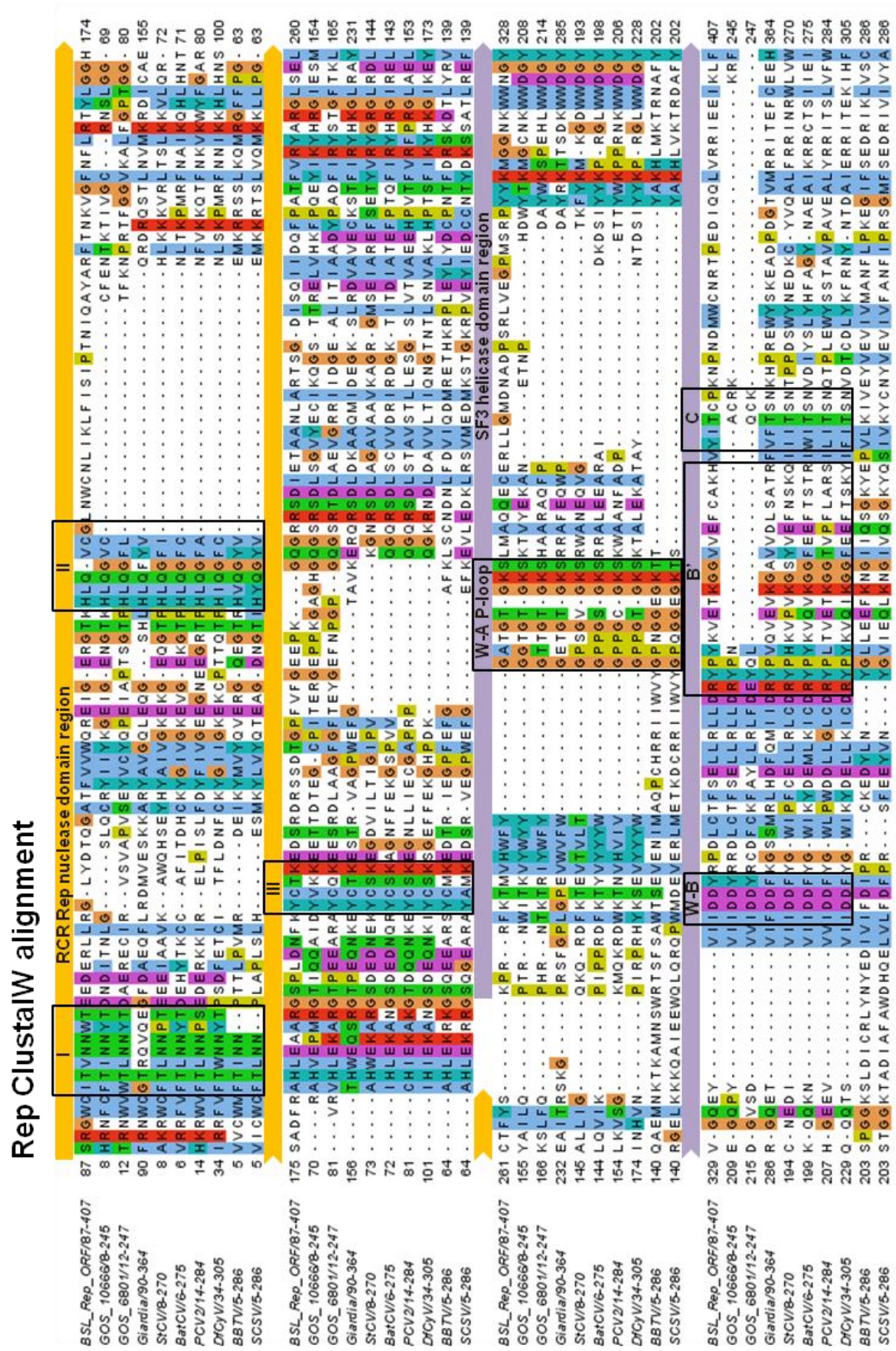


Figure 3.4 description:

The BSL RDHV Rep ORF sequence (BSL_Rep_ORF) is aligned to closely related sequences retrieved from NCBI using PSI-BLAST searches. Sequence alignment was performed using ClustalW set to default parameters in MEGA v.5. The endonuclease domain motifs I, II and III, and Superfamily-3 helicase domain Walker A P-loop NTPase (W-A P-loop), Walker B (W-B), B' and C motifs are boxed and labeled. Rep nuclease (RCRE) and Superfamily-3 helicase (S3H helicase) domains are indicated by block arrows above the sequence alignment. A ClustalW color scheme is applied to the multiple alignment using Jalview. The range of amino acid residues used in the alignment are shown after the sequence name. (GOS) Global Ocean Survey paired-end read scaffolds 10666 and 6801. CIRCOVIRIDAE: (PCV2) Porcine Circovirus-2, (StCV) Starling Circovirus, (BatCV) Bat Circovirus, (DfCyV) Dragonfly Cyclovirus. NANOVIRIDAE: (BBTV) Banana Bunchy Top Virus, (SCSV) Subterranean Clover Stunt Virus. 
Figure 3.5 BLASTp data for capsid protein (CP) amino acid sequences

\begin{tabular}{|c|c|c|c|c|c|c|c|}
\hline \multirow{5}{*}{ DNA } & \multicolumn{7}{|c|}{ BLASTp RESULTS FOR CAPSID PROTEINS } \\
\hline & Accession & Description & Max score & Total score & Query coverage & Evalue & Max ident \\
\hline & & \multicolumn{2}{|l|}{ Global Ocean Survey } & & & & \\
\hline & 142008898 & GOS_10665 & 181 & 229 & $75 \%$ & $2.0 \mathrm{E}-54$ & $86 \%$ \\
\hline & 134313055 & GOS_6800 & 174 & 203 & $58 \%$ & $4.0 \mathrm{E}-54$ & $39 \%$ \\
\hline \multirow[t]{3}{*}{ SsRNA } & & Nodavirus-like & & & & & \\
\hline & 45476497 & SmV-A & 108 & 125 & $44 \%$ & $5.0 \mathrm{E}-30$ & $56 \%$ \\
\hline & 301070442 & PhV-A & 106 & 121 & $41 \%$ & $2.0 \mathrm{E}-29$ & $31 \%$ \\
\hline \multirow[t]{16}{*}{ Tombusviridae } & & Tombusvirus & & & & & \\
\hline & 38680523 & HaRV & 51.2 & 70.1 & $54 \%$ & $3.0 \mathrm{E}-11$ & $42 \%$ \\
\hline & 230755 & TBSV_2TBV-A & 41.2 & 73.5 & $38 \%$ & 4.0E-08 & $47 \%$ \\
\hline & & Avenavirus & & & & & \\
\hline & 20177494 & OCSV & 52 & 72.4 & $75 \%$ & $2.0 \mathrm{E}-11$ & $32 \%$ \\
\hline & & Carmovirus & & & & & \\
\hline & 209156392 & MNSV_2ZAH-A & 52.8 & 68.9 & $36 \%$ & $6.0 \mathrm{E}-12$ & $42 \%$ \\
\hline & 9629190 & SgCV & 29.6 & 29.6 & $35 \%$ & $1.0 \mathrm{E}-04$ & $24 \%$ \\
\hline & 374977874 & TCV_3ZXA-C & 28.1 & 106 & $40 \%$ & 4.0E-04 & $54 \%$ \\
\hline & 29726963 & CMtV_1OPO-C & 25.8 & 58.5 & $41 \%$ & $2.0 \mathrm{E}-03$ & $67 \%$ \\
\hline & & Dianthovirus & & & & & \\
\hline & 20428582 & RCNMV & 33.9 & 48.1 & $30 \%$ & 7.0E-06 & $56 \%$ \\
\hline & 304452 & CRSV & 24.6 & 24.6 & $27 \%$ & $5.0 \mathrm{E}-03$ & $23 \%$ \\
\hline & & Satellite virus & & & & & \\
\hline & 75766385 & STNV & 16.2 & 31.6 & $8 \%$ & $1.1 \mathrm{E}+00$ & $36 \%$ \\
\hline & 20177432 & SV-MWLMV & 15.4 & 15.4 & $3 \%$ & $2.1 \mathrm{E}+00$ & $29 \%$ \\
\hline \multirow{10}{*}{ circular ssDNA } & & Nanovirus & & & & & \\
\hline & 20530235 & SCSV & 17.7 & 59.3 & $16 \%$ & 2.9E-01 & $38 \%$ \\
\hline & 118490329 & BBTV & 13.5 & 26.6 & $7 \%$ & $7.1 \mathrm{E}+00$ & $33 \%$ \\
\hline & & Geminivirus & & & & & \\
\hline & 167380550 & HrCTV & 17.7 & 62 & $7 \%$ & 0.49 & $63 \%$ \\
\hline & & Circovirus & & & & & \\
\hline & 190410825 & PCV2 & 19.2 & 33.5 & $6 \%$ & 1.5E-01 & $33 \%$ \\
\hline & 81176722 & StCV & 16.9 & 49.6 & $13 \%$ & $1.0 \mathrm{E}+00$ & $50 \%$ \\
\hline & & Cyclovirus & & & & & \\
\hline & 324309815 & DfCyV & 15.4 & 28.9 & $3 \%$ & $2.5 E+00$ & $60 \%$ \\
\hline
\end{tabular}

The BSL RDHV CP ORF amino acid sequence was compared to related CP sequences using BLASTp. Output parameters are shown. Virus family designations are indicated when possible. (GOS) Global Ocean Survey paired-end read sequences 10665 and 6800. (SmV-A) Sclerophthora macrospora Virus-A, (PhV-A) Plasmopara halstedii Virus-A. (HaRV) Havel River Tombusvirus, (TBSV) Tomato Bushy Stunt Virus, (OCSV) Oat Chlorotic Stunt Virus, (MNSV) Melon Necrotic Spot Virus, (SgCV) Saguaro Cactus Virus, (TCV) Turnip Crinkle Virus, (CMtV) Carnation Mottle Virus, (RCNMV) Red Clover Necrotic Mosaic Virus, (CRSV) Carnation Ringspot Virus, (STNV) Satellite of Tobacco Necrosis Virus, (SV-MWLMV) Satellite Virus of Maize White Line Mosaic Virus, (SCSV) Subterranean Clover Stunt Virus, (BBTV) Banana Bunchy Top Virus, (HrCTV) Horseradish Curly Top Virus, (PCV2) Porcine Circovirus-2, (StCV) Starling Circovirus, (DfCyV) Dragonfly Cyclovirus. 
Figure 3.6 Capsid protein (CP) amino acid multiple sequence alignment

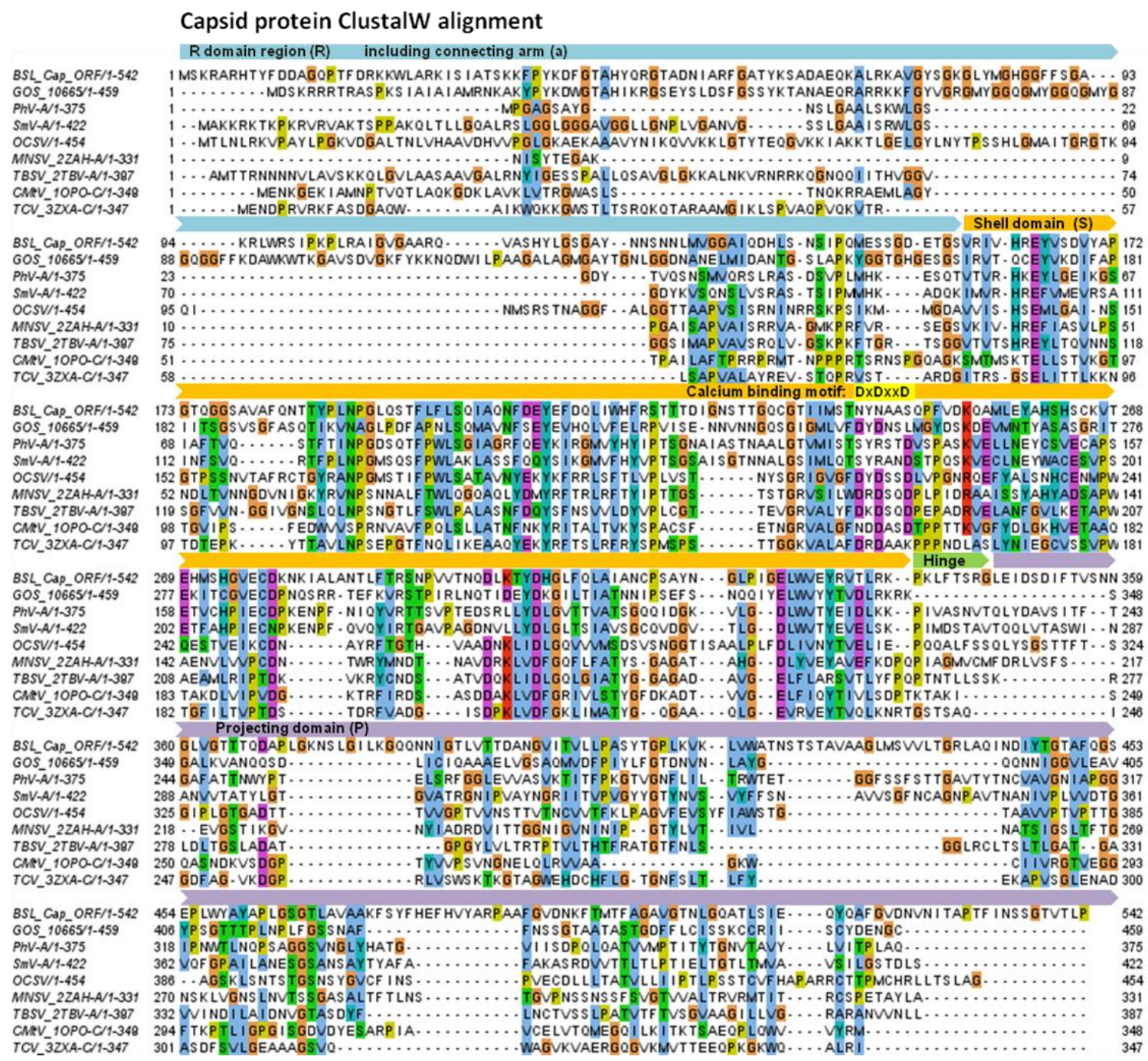

The BSL RDHV CP ORF sequence (BSL_Cap_ORF) is aligned to closely related sequences retrieved from PSI-BLAST searches using ClustalW set to default parameters in MEGA v.5. The R, a, S, h and P domain regions are indicated by block arrows above the sequence alignment. A ClustalW color scheme is applied to the multiple alignment using Jalview. (GOS) Global Ocean Survey paired-end read scaffold 10665.

NODAVIRIDAE-like: (PhV-A) Plasmopara halstedii Virus-A, (SmV-A) Sclerophthora macrospora Virus-A. TOMBUSVIRIDAE: Avenavirus; (OCSV) Oat Chlorotic Stunt Virus. Carmoviruses; (MNSV) Melon Necrotic Spot Virus, (CMtV) Carnation Mottle Virus, (TCV) Turnip Crinkle Virus. Tombusvirus; (TBSV) Tomato Bushy Stunt Virus. The range of amino acid residues used in the alignment are shown after the sequence name. Virus proteins for which structures are available are listed with the Protein Data Bank (PDB) identification code after the virus abbreviation. 
Figure 3.7 BSL RDHV predicted protein structures
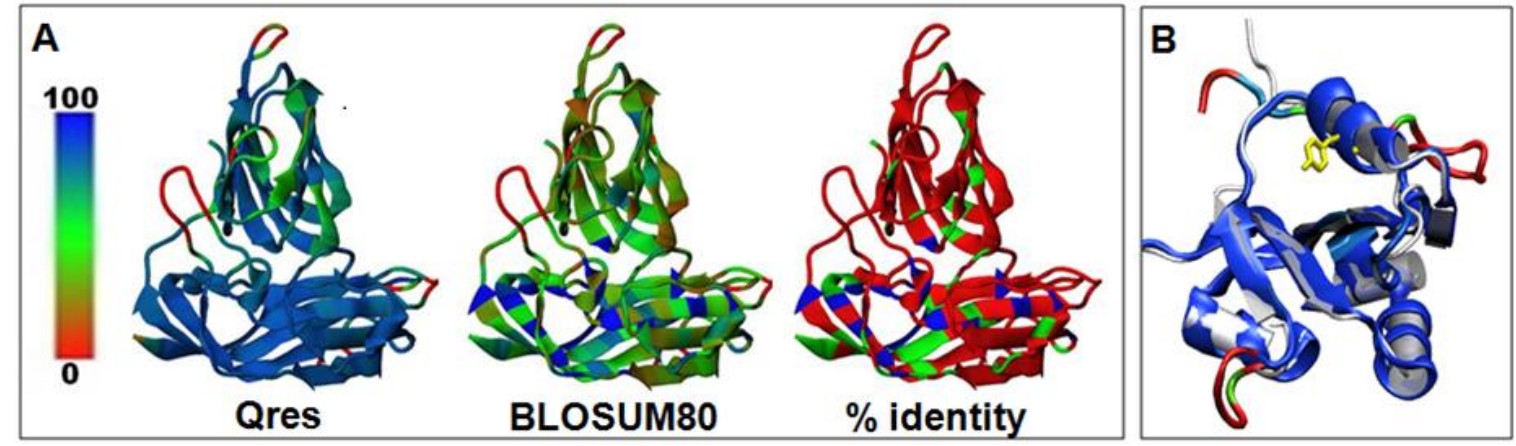

(A) Structural analysis of the BSL RDHV capsid protein: The predicted structure for residues 156-302 of the BSL RDHV capsid protein monomer ORF is overlaid on the Tomato Bushy Stunt Virus (PDB ID: 2TBV) and Melon Necrotic Spot Virus (PDB ID: 2ZAH) capsid proteins (latter two are not shown). The predicted BSL RDHV capsid is color-coded by the "Qres" dimensional structure-fitting parameter score, by BLOSUM80, and by percent amino acid sequence identity. (B) Structural analysis of the BSL RDHV replicase catalytic core: The predicted BSL RDHV Rep nuclease domain structure (colored by Qres) threaded onto PCV2 Rep (PDB ID: 2HW0) in silver. The conserved active-site tyrosine is shown in yellow. 


\section{CHAPTER 4: Synopsis and Discussion}

The advantages of sequencing low-complexity virus assemblages:

The science of metagenomics is greatly expanding our understanding and appreciation of the viral universe. The analyses conducted herein demonstrate just a few of the many ways in which viral metagenomic sequence data may be utilized to not only evaluate the composition of natural virus assemblages, but to discover new viral genes and interpret viral "dark matter" (CHAPTER 1), and to better understand the dynamics of both structural and genomic evolution [64, 69, 114, 183-186] (CHAPTERS 2 and 3).

Metagenomic DNA samples derived from rich and highly diverse virus communities are often too complex to be thoroughly or completely sequenced $[90,186]$. Samples that are not sequenced thoroughly generate fragmentary data that is difficult to assemble into large sections of the original viral genomes. Given the extensive genetic and genomic variability often observed even within the same virus group [183], and interviral horizontal gene transfer $[66,115]$, virus ecologists must carefully consider the validity of determining the taxonomic composition of a natural virus assemblage based solely on single genes or gene fragments.

Conversely, low-complexity samples can be sequenced more thoroughly, resulting in sequence read data that can be assembled into complete, or near complete genomes $[156,187]$. The Boiling Springs Lake (BSL) assemblage proved to be relatively low in diversity and sequencing of the metagenome was, as a result, quite thorough. The 
BSL sample was also, in effect, sequenced twice, as the data from two separate $\varphi 29$ amplifications of the metagenomic DNA were pooled prior to contig assembly (CHAPTER 1). This low diversity and duplicate sequencing allowed for large contigs, representing complete viral genomes and large genomic fragments, to be assembled at high stringency. Furthermore, 2,073 complete and large open reading frames predicted from these contigs could be characterized by comparison to reference databases and then mapped back to their contigs of origin. This provided a contextual basis for charting both known and uncharacterized components of BSL virus sequence-space, and for determining the genomic and genotypic similarity between well-characterized viruses and those found in BSL (CHAPTER 1).

The BSL virus assemblage harbors few "genera", but many "species":

Automated taxonomic assessment [26] and PHACCS analysis [27] both indicated that the BSL assemblage was comprised of a low diversity of virus groups (Figure 1.1). Overall, the viral gene content of BSL most closely resembles what is found in other extreme environments; namely acid mine drainage, hypersaline and hydrothermal sites, and qualitatively supports the premise that the types of viruses that comprise the BSL assemblage are, to some extent, determined by the physiochemical characteristics of the environment itself $[54,188]$.

A high degree of sequence heterogeneity and genomic synteny was also observed within several of the of homologous genes and contigs, such that some of the identifiable 
virus groups found in BSL (based on both genetic and contig similarity to known virus genes and genomes) appear to be represented by several distinct strains. As the BSL metagenomic reads could readily be assembled at very high stringency into large contigs, it was possible to observe this intraspecific diversity directly through rarefaction clustering and through the alignment of individual homologous contigs and ORFs (Figure 1.3 and microviruses discussed in CHAPTER 2).

Comparative rarefaction analysis of the BSL metavirome data, as well as data from several other published viral metagenomes, demonstrated that BSL exhibits the highest degree of intraspecific or strain diversity among the metagenomes. The read-percluster ratios (i.e., the average number of homologous sequences binned into each cluster) were found to be strongly inversely proportional to rarefaction stringency in metagenomes derived from oligotrophic environments (Figure 1.3). Sequence reads from highly diverse virus assemblages or under-sequenced metagenomes, on the other hand, were largely unique and were not binned into homologous clusters even as alignment stringencies were reduced. Thus, low $\alpha$-diversity (a relatively small number of virus groups) coupled with high intraspecific or strain diversity is an interrelated set of traits that may only be detectible in thoroughly-sequenced, low-diversity assemblages.

These data further indicate that this diversity pattern may be associated with the trophic state of the system as well. However, further extensive analysis, comparing datasets derived from environments whose trophic states have been well characterized, would be required to substantiate this hypothesis. If such an experiment were attempted, all of the samples (whether low or high diversity) would need to be thoroughly 
sequenced. New techniques for artificially reducing the intrinsic complexity of an assemblage have recently been developed, which allow for fractionation of viruses in the sample assemblage prior to sequencing $[62,189,190]$. Each sub-fraction of a highdiversity assemblage can then be sequenced separately, making thorough sequencing possible and reducing the computational power and resources required for analysis.

Low-complexity facilitates the charting of unknown virus sequence-space:

Another key advancement, stemming from the low-diversity BSL sample, was the ability to more precisely estimate the nature and extent of ORFan sequences in the assemblage. These ORFans, having no identifiable homologs in the annotated sequence databases, are often disregarded in the analysis of metaviromes, and may speciously suggest that an inordinately large number of virus types have yet to be discovered [191]. The context provided by efficient assembly of the BSL data made it possible to precisely estimate the origin and distribution of ORFans throughout the assemblage (Figures 1.6 and 1.7). This was achieved by categorizing the ORFans based on their co-localization with known viral genes within the same contig. Results of the comparative and protein rarefaction clustering analyses indicate that novel ORFans dominate the BSL virus assemblage, and appear to be largely endemic to this particular environment. However, it is not yet known how (or how rapidly) these novel genes are created, whether viruses or host cells are creating them $[57,66]$, or whether they contribute in any way to the adaptive capabilities of the microbial ecosystem [10, 57, 66-69] 
Homologs of BSL ORFans were also identified in other metagenomic datasets using a comparative genomics approach [192]. Conserved protein sequences of unknown function identified in another metagenomic dataset are referred to herein as "gray matter" ORFans. This analysis identified 3\% of the 2,073 BSL metavirome ORFs as "gray matter" ORFans. Many of the true ("dark") and gray matter ORFans were also found to be located in contigs containing known virus genes, indicating that much of the "dark matter of viral sequence space" [29] may thus be harbored by known virus families, as opposed to representing new taxa (Figures 1.5 and 1.6).

The use of comparative metagenomic analysis to identify common gray matter ORFans may also aid in future bioprospecting efforts [64, 193-195]. For example, the search for thermostable viral enzymes could be expedited by first surveying hydrothermal and terrestrial hot spring environments for gray matter ORFan homologs (Figure 1.5). Once identified, they may be isolated from the environment for detailed functional characterization in the laboratory. The comparative metagenomic analysis of BSL ORFans resulted in the identification of 17 uncharacterized high-temperature environment-specific genes that may be worthy of further investigation (Figure 1.8).

Metagenomic sequence data enhances our understanding of virus protein family diversity and evolution:

Metagenomic data is also being used to better chart the sequence space of known protein families as well [196-198]. For example, once a protein sequence is predicted 
from metavirome data, and further identified as being a homolog of a known virus gene, this new data can be utilized to augment what is currently known about the evolutionary history and diversity of the protein family [199]. Many comparisons may be made on the basis of sequence alignment alone, however, the tales of protein evolution are often best told through detailed structural analyses [200].

Comprehensive sequencing of the low-diversity BSL virus assemblage enabled the identification and isolation of two distinct and complete major capsid protein (MCP) sequences from the globally-ubiquitous Gokushovirinae sub-family of microviruses (CHAPTER 2). Fortunately, much is already known about the structure of MCPs within the Microviridae family $[79,80]$, thus metagenomic sequence data from BSL and other metagenomes were utilized to evaluate the scope of diversity within this family of viral proteins, and to garner new insights into how these proteins may have evolved and adapted to different environments. While similar analyses have been done utilizing metagenomic data [201-203], none are on a comparable scale to the study described herein, or have focused specifically on the structural dynamics of virus protein evolution.

A recent explosion of microvirus MCP sequence data from metagenomic surveys has offered an unprecedented opportunity to conduct an exhaustive structure-based analysis of this protein family [45]. Structure-based sequence analysis demonstrated that inferred conservative and semi-conservative nonsynonymous substitutions largely appear to be associated with internal secondary structures within each MCP, and that radical nonsynonymous substitutions are prevalent in the tri-fold loop protrusion (Figures $\mathbf{2 . 5}$ and 2.8). Strict conservation of sequence identity throughout this diverse protein family, 
however, was not determined to be strongly correlated with secondary or tertiary structures within the protein. Instead, the most strictly conserved amino acids spanning the largest number of taxa appear to be localized to regions involved in protein-protein interactions required for assembly of the virion (Figures 2.6, 2.7 and2.8). Surprisingly, many of the residues that are strictly conserved among smaller groups of more closely related taxa appear to form banded "girder"-like structures which extend laterally along the entire length of MCP dimers within the virion (Figure 2.8). Being that this pattern of conservation has never before been observed in this group of viral proteins, it is not known what function these conserved structures may serve.

Another phenomenon observed in both of the BSL gokushoviruses is an overall increase in the isoelectric point (pI) of the major capsid protein, relative to all other gokushovirus MCPs from circum-neutral environments (Table 2.2). The specific reason for this difference is not entirely clear, however, it is tempting to speculate that it is an adaptation to the highly acidic conditions of the BSL environment. A worthwhile and feasible future research endeavor, therefore, might involve evaluating all capsid protein sequences detected within BSL and other acidic environments, to assess whether increased $\mathrm{pI}$ is an attribute common to all viruses in acidic environments.

Genomic context analysis expands the potential modalities of virus evolution:

Genomic context analysis, again made possible by thorough sequencing of the BSL sample and the efficient assembly of contigs, revealed a unique interviral gene 
transfer event having transpired between highly disparate single-stranded (ss)RNA and ssDNA virus groups. Two large open reading frames were predicted within a single BSL contig roughly $4 \mathrm{~kb}$ in length, and subsequently determined by sequence comparison to be related to genes from very different virus groups. One of these genes is only known to exist in RNA viruses which completely lack a DNA stage in their life-cycle. However, the other gene is only found in ssDNA virus groups. This strange juxtaposition of unrelated viral genes implied that a circular ssDNA virus apparently acquired a capsid protein $(\mathrm{CP})$ gene from a linear ssRNA virus, presumably during co-infection of the same host cell (Figure 3.1). This seemingly unlikely gene transfer event resulted in the formation of a novel virus genome referred to herein as an RNA-DNA Hybrid Virus (RDHV).

The discovery of the BSL RDHV genome, and three RDHV-like scaffold sequences in the Global Ocean Survey database [34] (CHAPTER 3), prompted several other researchers to engage in a concerted search for similar viruses in different environments. As several other surveys of DNA metagenomes have also uncovered evidence of RNA virus sequences $[38,42]$, it seemed likely that the sequence reads in these datasets were fragmentary and not capable of being assembled into long contigs, thus making it impossible to observe whether these RNA virus sequences were associated with genes from DNA viruses. Recently however, putative RDHV-like genomes have been identified in freshwater lake cultures of Daphnia mendotae [41], the marine / estuarine waters of Tampa Bay, Florida [204], and from a search of 103 separate metagenomic datasets and whole genome shotgun libraries [205]. It is not yet known, 
however, whether the RDHV virus genomes found thus far represent a monophyletic group, or whether similar gene transfer events have transpired numerous times between ssDNA and ssRNA viruses of the same general type to create a polyphyletic group of RDHV-like genomes.

One reason to suggest a polyphyletic lineage is that the Rep and capsid protein genes found within the RDHV-like group occur in both uni- and ambisense orientations $[204,205]$. If the group were monophyletic, one might expect to find these genes oriented exclusively in the unisense orientation found in the original BSL-RDHV. Additionally, a genome encoding a circovirus-like Rep and a capsid protein gene exhibiting weak but significant similarity to that of the Satellite Tobacco Necrosis RNA virus, was recently found in a dragonfly metavirome [110]. As this RNA virus-like CP lacks detectable similarity to the RDHV CP protein sequence, it suggests that Rep-encoding ssDNA viruses are capable of acquiring cistrons from other types of RNA viruses (and perhaps host-derived mRNAs) and incorporating them into their genomes.

However, there is also evidence to support a monophyletic lineage. For example, of the 13 genomes found by Roux et. al., [205] five have circovirus-like Reps, seven have nanovirus-like Reps and one has a Rep most closely related to those found in geminiviruses. Interestingly, all of the capsid proteins harbored by these viruses, however, do not appear to have diverged significantly. This implies that the ancestral capsid protein was acquired once by a plasmid or ssDNA virus, resulting in the first RDHV genome, and that the descendents of this original genome have subsequently 
obtained or exchanged homologous Rep genes from other closely related groups of circo-, nano- and geminiviruses by lateral gene transfer [205].

Whatever the case may be, the manner in which interviral recombination could have occurred between RNA and DNA viruses remains a mystery. One scenario suggests that Rep gene mRNA from a ssDNA virus was incorporated into a tombusvirus-like RNA genome, and was subsequently converted to DNA by reverse transcription to form the circular ssDNA genome [206]. However, the ligase and endonuclease activities of the ssDNA virus Rep protein implicates its possible involvement in the acquisition of RNA molecules during the infection phase [40]. Several interesting scenarios describing how interviral hybridization might have transpired have been proposed, including the possibility that Rep may have directly ligated two RNA and DNA molecules together during the rolling circle replication cycle [40]. While the ssDNA virus Rep protein is not known to directly ligate DNA and RNA molecules together, a precedent for this scenario derives from the Vaccinia poxvirus. Intronless host-derived genes, ostensibly acquired through the direct ligation of host mRNAs, have been detected at the terminal ends of the Vaccinia DNA genome [207]. The Vaccinia DNA ligase enzyme was subsequently shown to be capable of directly ligating RNA-DNA molecules together [208].

Whether RDHV was formed by the ligation of RNA and DNA molecules or not, all hybridization scenarios require that the RNA virus cistron be, at some point, reverse transcribed into DNA. One possible explanation for how this might have occurred is that the ancestral RNA virus also encoded an reverse transcriptase (RT)-like enzyme, or that a retrovirus was also co-infecting the cell at the time of genome hybridization. Reverse 
transcription of the ssRNA virus $\mathrm{CP}$ cistron, however, could have occurred before the gene was acquired by the ssDNA virus [206], as most cells also possess non-viral reverse transcriptase activity [209]. Thus a parsimonious scenario would be that host-derived RT machinery was responsible for reverse-transcribing the RNA CP cistron, and that genomic hybridization resulted from the ligation of two DNA molecules following reverse transcription of the ssRNA cistron into DNA.

Furthermore, simultaneous co-infection with RNA and DNA viruses may not have even been necessary for the hybridization to occur. Endogenous non-retroviral RNA sequences have been found embedded in the genomes of plants and fungi [165-167], thus it is conceivable that the capsid protein gene originated from an ancestral tombusvirus that had been integrated into the genome of the host organism. As Rep sequences and whole genomes from ssDNA viruses and plasmids have also been found integrated into the genomes of bacteria [77] and many different eukaryotic organisms [155, 169, 210], the hybridization event(s) that formed RDHV may have occurred in hosts harboring endogenous forms of these viruses.

While many intriguing hypotheses have been proposed, it is likely that only painstaking laboratory research will reveal the mechanism which generated the RDHV. Regardless, the discovery of this unique virus genome in BSL has substantially expanded our understanding of viral genomic evolution. 


\section{Concluding remarks and commentary:}

The science of metagenomics continues to revolutionize the field of virus ecology, as thousands of novel viruses and viral genes are discovered in the environment. However, metagenomic data can reveal far more than a mere inventory of viruses present within an ecosystem. The body of work presented herein demonstrates how metagenomic data can also be used to chart the dark, unknown recesses of virus sequence-space, to discover new viruses and viral genes, and to better understand the dynamics of structural and genomic evolution in the virosphere. The potential insights to be gained, however, are reliant upon the quality of the sequence data produced, the volume of annotated reference sequence data in the databases, and the repertoire of tools that are created to efficiently process and analyze the vast amount of data produced by metagenomic surveys. The critical importance of thorough sequencing, which is facilitated by the acquisition of a low-complexity sample, is becoming better appreciated as the field of metagenomics matures and evolves. Future technological advancements in the field of viral metagenomics will undoubtedly foster a better understanding and appreciation of the enigmatic world of viruses and their importance within the biosphere. 


\section{REFERENCES}

1. Sorey, M.L., E.M. Colvard, and Geological Survey (U.S.), Measurements of heat and mass flow from thermal areas in Lassen Volcanic National Park, California, 1984-93. Water-resources investigations report. 1994, Menlo Park, Calif. and Denver, Colo.: U.S. Geological Survey, USGS Earth Science Information Center, Open-File Reports Section distributor. iii, 35 p.

2. Siering, P.L., et al., Microbial biogeochemistry of Boiling Springs Lake: a physically dynamic, oligotrophic, low-pH geothermal ecosystem. Geobiology, 2013. 11(4): p. 356-76.

3. Brown, P.B. and G.V. Wolfe, Protist genetic diversity in the acidic hydrothermal environments of Lassen Volcanic National Park, USA. J Eukaryot Microbiol, 2006. 53(6): p. 420-31.

4. Wilson, M.S., et al., Novel archaea and bacteria dominate stable microbial communities in North America's Largest Hot Spring. Microb Ecol, 2008. 56(2): p. 292-305.

5. Geological Survey of California. and J.D. Whitney, Geology. 1865, Philadelphia, Caxton Press of Sherman and Co. v. $<1>$.

6. Lloyd, E.F. and R.F. Keam, Waimangu Geology In: New Zealand volcanology, central volcanic region; New Zealand Geological Survey handbook. New Zealand Dept of Scientific and Industrial Research Information series,, ed. B.N.

Thompson, et al. 1965, Wellington: New Zealand Dept. of Scientific and Industrial Research. 211 p.

7. Danovaro, R., et al., Major viral impact on the functioning of benthic deep-sea ecosystems. Nature, 2008. 454(7208): p. 1084-7.

8. Suttle, C.A., Marine viruses--major players in the global ecosystem. Nat Rev Microbiol, 2007. 5(10): p. 801-12.

9. Jiao, N., et al., Microbial production of recalcitrant dissolved organic matter: long-term carbon storage in the global ocean. Nat Rev Microbiol, 2010. 8(8): p. 593-9.

10. Rohwer, F. and R.V. Thurber, Viruses manipulate the marine environment. Nature, 2009. 459(7244): p. 207-12.

11. Dinsdale, E.A., et al., Functional metagenomic profiling of nine biomes. Nature, 2008. 452(7187): p. 629-32.

12. Williamson, S.J., et al., Lysogenic virus-host interactions predominate at deepsea diffuse-flow hydrothermal vents. ISME J, 2008. 2(11): p. 1112-21.

13. Ackermann, H.-W. and M.S. DuBow, Viruses of prokaryotes. 1987, Boca Raton, Fla.: CRC Press.

14. Goldenfeld, N. and C. Woese, Biology's next revolution. Nature, 2007. 445(7126): p. 369.

15. Koonin, E.V., Taming of the shrewd: novel eukaryotic genes from RNA viruses. BMC Biol, 2010. 8: p. 2.

16. Koonin, E.V., T.G. Senkevich, and V.V. Dolja, The ancient Virus World and evolution of cells. Biol Direct, 2006. 1: p. 29. 
17. Forterre, P., The two ages of the RNA world, and the transition to the DNA world: a story of viruses and cells. Biochimie, 2005. 87(9-10): p. 793-803.

18. Forterre, P., The origin of viruses and their possible roles in major evolutionary transitions. Virus Res, 2006. 117(1): p. 5-16.

19. Ortmann, A.C., et al., Hot crenarchaeal viruses reveal deep evolutionary connections. Nat Rev Microbiol, 2006. 4(7): p. 520-8.

20. Prangishvili, D., et al., Structural and genomic properties of the hyperthermophilic archaeal virus ATV with an extracellular stage of the reproductive cycle. J Mol Biol, 2006. 359(5): p. 1203-16.

21. Diemer, G., J. Kyle, and K. Stedman, Counting viruses using polycarbonate Track Etch ${ }^{\mathrm{TM}}$ membrane filters as an alternative to Anodisc ${ }^{\mathrm{TM}}$ membrane filters. 2012, Portland State University: http://web.pdx.edu/ kstedman/PCTE_virus_counting_protocol.pdf.

22. Hurwitz, B.L., et al., Evaluation of methods to concentrate and purify ocean virus communities through comparative, replicated metagenomics. Environ Microbiol, 2013. 15(5): p. 1428-40.

23. Bench, S.R., et al., Metagenomic characterization of Chesapeake Bay virioplankton. Appl Environ Microbiol, 2007. 73(23): p. 7629-41.

24. Li, W., J.C. Wooley, and A. Godzik, Probing metagenomics by rapid cluster analysis of very large datasets. PLoS One, 2008. 3(10): p. e3375.

25. Angly, F.E., et al., The marine viromes of four oceanic regions. PLoS Biol, 2006. 4(11): p. e368.

26. Roux, S., et al., Metavir: a web server dedicated to virome analysis. Bioinformatics, 2011. 27(21): p. 3074-5.

27. Angly, F., et al., PHACCS, an online tool for estimating the structure and diversity of uncultured viral communities using metagenomic information. BMC Bioinformatics, 2005. 6: p. 41.

28. Sun, S., et al., Community cyberinfrastructure for Advanced Microbial Ecology Research and Analysis: the CAMERA resource. Nucleic Acids Res, 2011. 39(Database issue): p. D546-51.

29. Pedulla, M.L., et al., Origins of highly mosaic mycobacteriophage genomes. Cell, 2003. 113(2): p. 171-82.

30. Fischer, D. and D. Eisenberg, Finding families for genomic ORFans. Bioinformatics, 1999. 15(9): p. 759-62.

31. Toplin, J.A., et al., Biogeographic and phylogenetic diversity of thermoacidophilic cyanidiales in Yellowstone National Park, Japan, and New Zealand. Appl Environ Microbiol, 2008. 74(9): p. 2822-33.

32. Meyer, F., et al., The metagenomics RAST server - a public resource for the automatic phylogenetic and functional analysis of metagenomes. BMC Bioinformatics, 2008. 9: p. 386.

33. Schoenfeld, T., et al., Assembly of viral metagenomes from yellowstone hot springs. Appl Environ Microbiol, 2008. 74(13): p. 4164-74. 
34. Diemer, G.S. and K.M. Stedman, A novel virus genome discovered in an extreme environment suggests recombination between unrelated groups of RNA and DNA viruses. Biol Direct, 2012. 7: p. 13.

35. Roux, S., et al., Chimeric viruses blur the borders between the major groups of eukaryotic single-stranded 4 DNA viruses Nature Communications, In Press.

36. Rosario, K., et al., Metagenomic analysis of viruses in reclaimed water. Environ Microbiol, 2009. 11(11): p. 2806-20.

37. Rosario, K., S. Duffy, and M. Breitbart, Diverse circovirus-like genome architectures revealed by environmental metagenomics. J Gen Virol, 2009. 90(Pt 10): p. 2418-24.

38. Whon, T.W., et al., Metagenomic characterization of airborne viral DNA diversity in the near-surface atmosphere. J Virol, 2012. 86(15): p. 8221-31.

39. Yoshida, M., et al., Metagenomic analysis of viral communities in (hado)pelagic sediments. PLoS One, 2013. 8(2): p. e57271.

40. Stedman, K., Mechanisms for RNA capture by ssDNA viruses: grand theft RNA. J Mol Evol, 2013. 76(6): p. 359-64.

41. Hewson, I.N., Gabriel Li, WenFang LaBarre, Brenna A. Aguirre, Isabel Barbosa, Jorge G. Breitbart, Mya Greco, Anthony W. Kearns, Colleen M. Looi, Alexander Schaffner, Lindsay R. Thompson, Philip D. Hairston, Nelson G., Jr.,

Metagenomic identification, seasonal dynamics, and potential transmission mechanisms of a Daphnia-associated single-stranded DNA virus in two temperate lakes. Limnology and Oceanography, 2013. 58(5): p. 1605-1620.

42. López-Bueno, A., et al., High diversity of the viral community from an Antarctic lake. Science, 2009. 326(5954): p. 858-61.

43. Hewson, I., et al., Investigation of viruses in Diporeia spp. from the Laurentian Great Lakes and Owasco Lake as potential stressors of declining populations. Journal of Great Lakes Research, 2013. 39(3): p. 499 - 506.

44. Roux, S., et al., Assessing the diversity and specificity of two freshwater viral communities through metagenomics. PLoS One, 2012. 7(3): p. e33641.

45. Roux, S., et al., Evolution and diversity of the Microviridae viral family through a collection of 81 new complete genomes assembled from virome reads. PLoS One, 2012. 7(7): p. e40418.

46. Pietilä, M.K., et al., An ssDNA virus infecting archaea: a new lineage of viruses with a membrane envelope. Mol Microbiol, 2009. 72(2): p. 307-19.

47. Pietilä, M.K., et al., The single-stranded DNA genome of novel archaeal virus halorubrum pleomorphic virus 1 is enclosed in the envelope decorated with glycoprotein spikes. J Virol, 2010. 84(2): p. 788-98.

48. Sencilo, A., et al., Related haloarchaeal pleomorphic viruses contain different genome types. Nucleic Acids Res, 2012. 40(12): p. 5523-34.

49. Breitbart, M., et al., Phage community dynamics in hot springs. Appl Environ Microbiol, 2004. 70(3): p. 1633-40.

50. Snyder, J.C., et al., Virus movement maintains local virus population diversity. Proc Natl Acad Sci U S A, 2007. 104(48): p. 19102-7. 
51. Tucker, K.P., et al., Diversity and distribution of single-stranded DNA phages in the North Atlantic Ocean. ISME J, 2011. 5(5): p. 822-30.

52. Emerson, J.B., et al., Dynamic viral populations in hypersaline systems as revealed by metagenomic assembly. Appl Environ Microbiol, 2012. 78(17): p. 6309-20.

53. Duffy, S., L.A. Shackelton, and E.C. Holmes, Rates of evolutionary change in viruses: patterns and determinants. Nat Rev Genet, 2008. 9(4): p. 267-76.

54. de Wit, R. and T. Bouvier, 'Everything is everywhere, but, the environment selects'; what did Baas Becking and Beijerinck really say? Environ Microbiol, 2006. 8(4): p. 755-8.

55. Srinivasiah, S., et al., Phages across the biosphere: contrasts of viruses in soil and aquatic environments. Res Microbiol, 2008. 159(5): p. 349-57.

56. Thurber, R.V., Current insights into phage biodiversity and biogeography. Curr Opin Microbiol, 2009. 12(5): p. 582-7.

57. Yin, Y. and D. Fischer, Identification and investigation of ORFans in the viral world. BMC Genomics, 2008. 9: p. 24.

58. Rohwer, F., Global phage diversity. Cell, 2003. 113(2): p. 141.

59. Hatfull, G.F., et al., Exploring the mycobacteriophage metaproteome: phage genomics as an educational platform. PLoS Genet, 2006. 2(6): p. e92.

60. Hurwitz, B.L. and M.B. Sullivan, The Pacific Ocean virome (POV): a marine viral metagenomic dataset and associated protein clusters for quantitative viral ecology. PLoS One, 2013. 8(2): p. e57355.

61. Duhaime, M.B. and M.B. Sullivan, Ocean viruses: rigorously evaluating the metagenomic sample-to-sequence pipeline. Virology, 2012. 434(2): p. 181-6.

62. Brum, J.R., A.I. Culley, and G.F. Steward, Assembly of a marine viral metagenome after physical fractionation. PLoS One, 2013. 8(4): p. e60604.

63. Cantalupo, P.G., et al., Raw sewage harbors diverse viral populations. MBio, 2011. 2(5).

64. Schoenfeld, T., et al., Functional viral metagenomics and the next generation of molecular tools. Trends Microbiol, 2010. 18(1): p. 20-9.

65. Schloss, P.D. and J. Handelsman, Biotechnological prospects from metagenomics. Curr Opin Biotechnol, 2003. 14(3): p. 303-10.

66. Yin, Y. and D. Fischer, On the origin of microbial ORFans: quantifying the strength of the evidence for viral lateral transfer. BMC Evol Biol, 2006. 6: p. 63.

67. Siew, N. and D. Fischer, Structural biology sheds light on the puzzle of genomic ORFans. J Mol Biol, 2004. 342(2): p. 369-73.

68. Sharon, I., et al., Comparative metagenomics of microbial traits within oceanic viral communities. ISME J, 2011. 5(7): p. 1178-90.

69. Rosario, K. and M. Breitbart, Exploring the viral world through metagenomics. Curr Opin Virol, 2011. 1(4): p. 289-97.

70. Blanco, L., et al., Highly efficient DNA synthesis by the phage phi 29 DNA polymerase. Symmetrical mode of DNA replication. J Biol Chem, 1989. 264(15): p. 8935-40. 
71. Lane, D., 16S/23S rRNA Sequencing, in Nucleic Acid Techniques in Bacterial Systematics, E. Stackebrandt and M. Goodfellow, Editors. 1991, Wiley: Chichester. p. 115-175.

72. Henn, M.R., et al., Analysis of high-throughput sequencing and annotation strategies for phage genomes. PLoS One, 2010. 5(2): p. e9083.

73. Altschul, S.F., et al., Gapped BLAST and PSI-BLAST: a new generation of protein database search programs. Nucleic Acids Res, 1997. 25(17): p. 3389-402.

74. Pruitt, K.D., et al., NCBI Reference Sequences (RefSeq): current status, new features and genome annotation policy. Nucleic Acids Res, 2012. 40(Database issue): p. D130-5.

75. Smoot, M.E., et al., Cytoscape 2.8: new features for data integration and network visualization. Bioinformatics, 2011. 27(3): p. 431-2.

76. Brentlinger, K.L., et al., Microviridae, a family divided: isolation, characterization, and genome sequence of phiMH2K, a bacteriophage of the obligate intracellular parasitic bacterium Bdellovibrio bacteriovorus. J Bacteriol, 2002. 184(4): p. 1089-94.

77. Krupovic, M. and P. Forterre, Microviridae goes temperate: microvirus-related proviruses reside in the genomes of Bacteroidetes. PLoS One, 2011. 6(5): p. e19893.

78. Thomas, V., N. Casson, and G. Greub, Criblamydia sequanensis, a new intracellular Chlamydiales isolated from Seine river water using amoebal coculture. Environ Microbiol, 2006. 8(12): p. 2125-35.

79. McKenna, R., et al., Atomic structure of single-stranded DNA bacteriophage phi X174 and its functional implications. Nature, 1992. 355(6356): p. 137-43.

80. Chipman, P.R., et al., Structural analysis of the Spiroplasma virus, SpV4: implications for evolutionary variation to obtain host diversity among the Microviridae. Structure, 1998. 6(2): p. 135-45.

81. Fane BA, B.K., Burch AD, Chen M, Hafenstein S, Moore E, Novak CR, Uchiyama A, the Microviridae, in The Bacteriophages, R. Calendar, Editor. 2006, Oxford University Press: Oxford ; New York. p. 129-145.

82. Liu, Y. and I. Bahar, Sequence evolution correlates with structural dynamics. Mol Biol Evol, 2012. 29(9): p. 2253-63.

83. Worth, C.L., S. Gong, and T.L. Blundell, Structural and functional constraints in the evolution of protein families. Nat Rev Mol Cell Biol, 2009. 10(10): p. 709-20.

84. Lee, K.H., et al., First-step mutations for adaptation at elevated temperature increase capsid stability in a virus. PLoS One, 2011. 6(9): p. e25640.

85. Crill, W.D., H.A. Wichman, and J.J. Bull, Evolutionary reversals during viral adaptation to alternating hosts. Genetics, 2000. 154(1): p. 27-37.

86. Bamford, D.H., R.M. Burnett, and D.I. Stuart, Evolution of viral structure. Theor Popul Biol, 2002. 61(4): p. 461-70.

87. Khayat, R., et al., Structure of an archaeal virus capsid protein reveals a common ancestry to eukaryotic and bacterial viruses. Proc Natl Acad Sci U S A, 2005.

102(52): p. 18944-9. 
88. Rice, G., et al., The structure of a thermophilic archaeal virus shows a doublestranded DNA viral capsid type that spans all domains of life. Proc Natl Acad Sci U S A, 2004. 101(20): p. 7716-20.

89. Benson, S.D., et al., Viral evolution revealed by bacteriophage PRD1 and human adenovirus coat protein structures. Cell, 1999. 98(6): p. 825-33.

90. Yooseph, S., et al., The Sorcerer II Global Ocean Sampling expedition: expanding the universe of protein families. PLoS Biol, 2007. 5(3): p. e16.

91. Poon, A. and L. Chao, The rate of compensatory mutation in the DNA bacteriophage phiX174. Genetics, 2005. 170(3): p. 989-99.

92. Cuevas, J.M., S. Duffy, and R. Sanjuán, Point mutation rate of bacteriophage PhiX174. Genetics, 2009. 183(2): p. 747-9.

93. Stern, A. and R. Sorek, The phage-host arms race: shaping the evolution of microbes. Bioessays, 2011. 33(1): p. 43-51.

94. Lipson, S.M. and G. Stotzky, Effect of proteins on reovirus adsorption to clay minerals. Appl Environ Microbiol, 1984. 48(3): p. 525-30.

95. Hsu, B.M., et al., Evaluation of enterovirus recovery in surface water by different adsorption and elution procedures. Chemosphere, 2007. 66(5): p. 964-9.

96. Goyal, S.M. and C.P. Gerba, Comparative adsorption of human enteroviruses, simian rotavirus, and selected bacteriophages to soils. Appl Environ Microbiol, 1979. 38(2): p. 241-7.

97. Dowd, S.E., et al., Delineating the specific influence of virus isoelectric point and size on virus adsorption and transport through sandy soils. Appl Environ Microbiol, 1998. 64(2): p. 405-10.

98. Gerba, C.P., Applied and theoretical aspects of virus adsorption to surfaces. Adv Appl Microbiol, 1984. 30: p. 133-68.

99. Sobsey, M.D., et al., Interactions and survival of enteric viruses in soil materials. Appl Environ Microbiol, 1980. 40(1): p. 92-101.

100. Needleman, S.B. and C.D. Wunsch, A general method applicable to the search for similarities in the amino acid sequence of two proteins. J Mol Biol, 1970. 48(3): p. 443-53.

101. Tamura, K., et al., MEGA5: molecular evolutionary genetics analysis using maximum likelihood, evolutionary distance, and maximum parsimony methods. Mol Biol Evol, 2011. 28(10): p. 2731-9.

102. Saitou, N. and M. Nei, The neighbor-joining method: a new method for reconstructing phylogenetic trees. Mol Biol Evol, 1987. 4(4): p. 406-25.

103. Bryson, V., H.J. Vogel, and Rutgers University. Institute of Microbiology., Evolving genes and proteins; a symposium held at the Institute of Microbiology of Rutgers, with support from the National Science Foundation. 1965, New York,: Academic Press. xxi, 629 p.

104. Petersen, T.N., et al., CPHmodels-3.0-remote homology modeling using structureguided sequence profiles. Nucleic Acids Research, 2010. 38: p. W576-W581.

105. Pei, J., M. Tang, and N.V. Grishin, PROMALS3D web server for accurate multiple protein sequence and structure alignments. Nucleic Acids Res, 2008. 36(Web Server issue): p. W30-4. 
106. Humphrey, W., A. Dalke, and K. Schulten, VMD: Visual molecular dynamics. Journal of Molecular Graphics, 1996. 14(1): p. 33-\&.

107. Walker, J.M., The proteomics protocols handbook. 2005, Totowa, N.J.: Humana Press. xviii, 988 p.

108. Dolinsky, T.J., et al., PDB2PQR: an automated pipeline for the setup of PoissonBoltzmann electrostatics calculations. Nucleic Acids Res, 2004. 32(Web Server issue): p. W665-7.

109. Dolinsky, T.J., et al., PDB2PQR: expanding and upgrading automated preparation of biomolecular structures for molecular simulations. Nucleic Acids Res, 2007. 35(Web Server issue): p. W522-5.

110. Rosario, K., et al., Diverse circular ssDNA viruses discovered in dragonflies (Odonata: Epiprocta). J Gen Virol, 2012. 93(Pt 12): p. 2668-81.

111. Felsenstein, J., Confidence Limits on Phylogenies: An Approach Using the Bootstrap. Evolution, 1985. 39(4): p. 783-791.

112. Suttle, C., Crystal ball. The viriosphere: the greatest biological diversity on Earth and driver of global processes. Environ Microbiol, 2005. 7(4): p. 481-2.

113. Fuhrman, J.A., Marine viruses and their biogeochemical and ecological effects. Nature, 1999. 399(6736): p. 541-548.

114. Kristensen, D.M., et al., New dimensions of the virus world discovered through metagenomics. Trends Microbiol, 2010. 18(1): p. 11-9.

115. Hambly, E. and C.A. Suttle, The viriosphere, diversity, and genetic exchange within phage communities. Curr Opin Microbiol, 2005. 8(4): p. 444-50.

116. Wu, D., et al., Stalking the fourth domain in metagenomic data: searching for, discovering, and interpreting novel, deep branches in marker gene phylogenetic trees. PLoS One, 2011. 6(3): p. e18011.

117. Moreira, D. and P. López-García, Ten reasons to exclude viruses from the tree of life. Nat Rev Microbiol, 2009. 7(4): p. 306-11.

118. Lopez, P. and E. Bapteste, Molecular phylogeny: reconstructing the forest. C R Biol, 2009. 332(2-3): p. 171-82.

119. Lima-Mendez, G., A. Toussaint, and R. Leplae, Analysis of the phage sequence space: the benefit of structured information. Virology, 2007. 365(2): p. 241-9.

120. Dolja, V.V. and E.V. Koonin, Common origins and host-dependent diversity of plant and animal viromes. Current Opinion in Virology, 2011. 1(5): p. 322-331.

121. Krupovic, M., et al., Genomics of bacterial and archaeal viruses: dynamics within the prokaryotic virosphere. Microbiol Mol Biol Rev, 2011. 75(4): p. 610-35.

122. Hendrix, R.W., Bacteriophage genomics. Curr Opin Microbiol, 2003. 6(5): p. 506-11.

123. Lefeuvre, P., et al., Widely conserved recombination patterns among singlestranded DNA viruses. J Virol, 2009. 83(6): p. 2697-707.

124. Martelli, G.P., et al., Family Flexiviridae: a case study in virion and genome plasticity. Annu Rev Phytopathol, 2007. 45: p. 73-100.

125. Monjane, A.L., et al., Recombination hotspots and host susceptibility modulate the adaptive value of recombination during maize streak virus evolution. BMC Evol Biol, 2011. 11: p. 350. 
126. Park, J., et al., Phylogenetic lineage of Tobacco leaf curl virus in Korea and estimation of recombination events implicated in their sequence variation. Virus Res, 2011. 159(2): p. 124-31.

127. Sztuba-Solińska, J., et al., RNA-RNA recombination in plant virus replication and evolution. Annu Rev Phytopathol, 2011. 49: p. 415-43.

128. Lima-Mendez, G., A. Toussaint, and R. Leplae, A modular view of the bacteriophage genomic space: identification of host and lifestyle marker modules. Res Microbiol, 2011. 162(8): p. 737-46.

129. Yokoi, T., S. Yamashita, and T. Hibi, The nucleotide sequence and genome organization of Sclerophthora macrospora virus A. Virology, 2003. 311(2): p. 394-9.

130. Heller-Dohmen, M., et al., The nucleotide sequence and genome organization of Plasmopara halstedii virus. Virol J, 2011. 8: p. 123.

131. Gorbalenya, A.E., E.V. Koonin, and Y.I. Wolf, A new superfamily of putative NTP-binding domains encoded by genomes of small DNA and RNA viruses. FEBS Lett, 1990. 262(1): p. 145-8.

132. Faurez, F., et al., Replication of porcine circoviruses. Virol J, 2009. 6: p. 60.

133. Mankertz, A., et al., Molecular biology of Porcine circovirus: analyses of gene expression and viral replication. Vet Microbiol, 2004. 98(2): p. 81-8.

134. Walker, S.L., R.S. Wonderling, and R.A. Owens, Mutational analysis of the adeno-associated virus Rep68 protein: identification of critical residues necessary for site-specific endonuclease activity. J Virol, 1997. 71(4): p. 2722-30.

135. Laufs, J., et al., Identification of the nicking tyrosine of geminivirus Rep protein. FEBS Lett, 1995. 377(2): p. 258-62.

136. Vega-Rocha, S., et al., Solution structure of the endonuclease domain from the master replication initiator protein of the nanovirus faba bean necrotic yellows virus and comparison with the corresponding geminivirus and circovirus structures. Biochemistry, 2007. 46(21): p. 6201-12.

137. Vega-Rocha, S., et al., Solution structure, divalent metal and DNA binding of the endonuclease domain from the replication initiation protein from porcine circovirus 2. J Mol Biol, 2007. 367(2): p. 473-87.

138. Vadivukarasi, T., K.R. Girish, and R. Usha, Sequence and recombination analyses of the geminivirus replication initiator protein. J Biosci, 2007. 32(1): p. 17-29.

139. Walker, S.L., R.S. Wonderling, and R.A. Owens, Mutational analysis of the adeno-associated virus type 2 Rep68 protein helicase motifs. J Virol, 1997. 71(9): p. 6996-7004.

140. Koonin, E.V., A common set of conserved motifs in a vast variety of putative nucleic acid-dependent ATPases including MCM proteins involved in the initiation of eukaryotic DNA replication. Nucleic Acids Res, 1993. 21(11): p. 2541-7.

141. James, J.A., et al., Crystal structure of the SF3 helicase from adeno-associated virus type 2. Structure, 2003. 11(8): p. 1025-35. 
142. Koonin, E.V., et al., The Big Bang of picorna-like virus evolution antedates the radiation of eukaryotic supergroups. Nat Rev Microbiol, 2008. 6(12): p. 925-39.

143. Wada, Y., et al., The structure of melon necrotic spot virus determined at $2.8 \mathrm{~A}$ resolution. Acta Crystallogr Sect F Struct Biol Cryst Commun, 2008. 64(Pt 1): p. 8-13.

144. Hopper, P., S.C. Harrison, and R.T. Sauer, Structure of tomato bushy stunt virus. $V$. Coat protein sequence determination and its structural implications. J Mol Biol, 1984. 177(4): p. 701-13.

145. Harrison, S.C., et al., Tomato bushy stunt virus at 2.9 A resolution. Nature, 1978. 276(5686): p. 368-73.

146. Olson, A.J., G. Bricogne, and S.C. Harrison, Structure of tomato busy stunt virus IV. The virus particle at 2.9 A resolution. J Mol Biol, 1983. 171(1): p. 61-93.

147. Dolja, V.V. and E.V. Koonin, Phylogeny of capsid proteins of small icosahedral RNA plant viruses. J Gen Virol, 1991. 72 ( Pt 7): p. 1481-6.

148. Campbell, J.W., et al., Calcium binding sites in tomato bushy stunt virus visualized by Laue crystallography. J Mol Biol, 1990. 214(3): p. 627-32.

149. Wu, Y.M., et al., Role of the DxxDxD motif in the assembly and stability of betanodavirus particles. Arch Virol, 2008. 153(9): p. 1633-42.

150. Laakso, M.M. and L.A. Heaton, Asp-->Asn substitutions in the putative calciumbinding site of the turnip crinkle virus coat protein affect virus movement in plants. Virology, 1993. 197(2): p. 774-7.

151. Rossmann, M.G., et al., Structural comparisons of some small spherical plant viruses. J Mol Biol, 1983. 165(4): p. 711-36.

152. Abbate, E.A., J.M. Berger, and M.R. Botchan, The X-ray structure of the papillomavirus helicase in complex with its molecular matchmaker E2. Genes Dev, 2004. 18(16): p. 1981-96.

153. Hickman, A.B., et al., Structural unity among viral origin binding proteins: crystal structure of the nuclease domain of adeno-associated virus Rep. Mol Cell, 2002. 10(2): p. 327-37.

154. Campos-Olivas, R., et al., The structure of a replication initiator unites diverse aspects of nucleic acid metabolism. Proc Natl Acad Sci U S A, 2002. 99(16): p. 10310-5.

155. Gibbs, M.J., et al., Two families of rep-like genes that probably originated by interspecies recombination are represented in viral, plasmid, bacterial, and parasitic protozoan genomes. Mol Biol Evol, 2006. 23(6): p. 1097-100.

156. Venter, J.C., et al., Environmental genome shotgun sequencing of the Sargasso Sea. Science, 2004. 304(5667): p. 66-74.

157. Hendrix, R.W., et al., Evolutionary relationships among diverse bacteriophages and prophages: all the world's a phage. Proc Natl Acad Sci U S A, 1999. 96(5): p. 2192-7.

158. Rokyta, D.R., et al., Horizontal gene transfer and the evolution of microvirid coliphage genomes. J Bacteriol, 2006. 188(3): p. 1134-42. 
159. Zoll, J., J.M. Galama, and F.J. van Kuppeveld, Identification of potential recombination breakpoints in human parechoviruses. J Virol, 2009. 83(7): p. 3379-83.

160. Shi, B.J., R.H. Symons, and P. Palukaitis, The cucumovirus $2 b$ gene drives selection of inter-viral recombinants affecting the crossover site, the acceptor RNA and the rate of selection. Nucleic Acids Res, 2008. 36(4): p. 1057-71.

161. White, K.A. and T.J. Morris, Recombination between Defective Tombusvirus Rnas Generates Functional Hybrid Genomes. Proceedings of the National Academy of Sciences of the United States of America, 1994. 91(9): p. 3642-3646.

162. Morse, M.A., A.C. Marriott, and P.A. Nuttall, The glycoprotein of Thogoto virus (a tick-borne orthomyxo-like virus) is related to the baculovirus glycoprotein GP64. Virology, 1992. 186(2): p. 640-6.

163. Gibbs, M.J. and G.F. Weiller, Evidence that a plant virus switched hosts to infect a vertebrate and then recombined with a vertebrate-infecting virus. Proc Natl Acad Sci U S A, 1999. 96(14): p. 8022-7.

164. Krupovic, M., J.J. Ravantti, and D.H. Bamford, Geminiviruses: a tale of a plasmid becoming a virus. BMC Evol Biol, 2009. 9: p. 112.

165. Taylor, D.J. and J. Bruenn, The evolution of novel fungal genes from nonretroviral RNA viruses. BMC Biol, 2009. 7: p. 88.

166. Klenerman, P., H. Hengartner, and R.M. Zinkernagel, A non-retroviral RNA virus persists in DNA form. Nature, 1997. 390(6657): p. 298-301.

167. Chiba, S., et al., Widespread endogenization of genome sequences of nonretroviral RNA viruses into plant genomes. PLoS Pathog, 2011. 7(7): p. e1002146.

168. Horie, M. and K. Tomonaga, Non-retroviral fossils in vertebrate genomes. Viruses, 2011. 3(10): p. 1836-48.

169. Liu, H., et al., Widespread horizontal gene transfer from double-stranded RNA viruses to eukaryotic nuclear genomes. J Virol, 2010. 84(22): p. 11876-87.

170. Zhuang, F., et al., Linear group II intron RNAs can retrohome in eukaryotes and may use nonhomologous end-joining for cDNA ligation. Proc Natl Acad Sci U S A, 2009. 106(43): p. 18189-94.

171. Brosius, J. and H. Tiedge, Reverse transcriptase: mediator of genomic plasticity. Virus Genes, 1995. 11(2-3): p. 163-79.

172. Villarreal, L.P. and G. Witzany, Viruses are essential agents within the roots and stem of the tree of life. J Theor Biol, 2010. 262(4): p. 698-710.

173. Botstein, D., A theory of modular evolution for bacteriophages. Ann N Y Acad Sci, 1980. 354: p. 484-90.

174. Hendrix, R.W., et al., The origins and ongoing evolution of viruses. Trends Microbiol, 2000. 8(11): p. 504-8.

175. Lasken, R.S. and T.B. Stockwell, Mechanism of chimera formation during the Multiple Displacement Amplification reaction. BMC Biotechnol, 2007. 7: p. 19.

176. Kumar, G., et al., Genomic DNA preparation using illustra GenomiPhi V2 and HY DNA Amplification kits. Nature Methods, 2007: p. An30-An32. 
177. Zuker, M., Mfold web server for nucleic acid folding and hybridization prediction. Nucleic Acids Res, 2003. 31(13): p. 3406-15.

178. Waterhouse, A.M., et al., Jalview Version 2--a multiple sequence alignment editor and analysis workbench. Bioinformatics, 2009. 25(9): p. 1189-91.

179. Quevillon, E., et al., InterProScan: protein domains identifier. Nucleic Acids Res, 2005. 33(Web Server issue): p. W116-20.

180. Finn, R.D., et al., The Pfam protein families database. Nucleic Acids Res, 2010. 38(Database issue): p. D211-22.

181. Lambert, C., et al., ESyPred3D: Prediction of proteins $3 D$ structures. Bioinformatics, 2002. 18(9): p. 1250-6.

182. Roberts, E., et al., MultiSeq: unifying sequence and structure data for evolutionary analysis. BMC Bioinformatics, 2006. 7: p. 382.

183. Edwards, R.A. and F. Rohwer, Viral metagenomics. Nat Rev Microbiol, 2005. 3(6): p. 504-10.

184. Delwart, E.L., Viral metagenomics. Rev Med Virol, 2007. 17(2): p. 115-31.

185. Bexfield, N. and P. Kellam, Metagenomics and the molecular identification of novel viruses. Vet J, 2011. 190(2): p. 191-8.

186. Wooley, J.C., A. Godzik, and I. Friedberg, A primer on metagenomics. PLoS Comput Biol, 2010. 6(2): p. e1000667.

187. Tyson, G.W., et al., Community structure and metabolism through reconstruction of microbial genomes from the environment. Nature, 2004. 428(6978): p. 37-43.

188. Dinsdale, E.A., et al., Multivariate analysis of functional metagenomes. Front Genet, 2013. 4: p. 41.

189. Solonenko, S.A. and M.B. Sullivan, Preparation of metagenomic libraries from naturally occurring marine viruses. Methods Enzymol, 2013. 531: p. 143-65.

190. Willner, D. and P. Hugenholtz, From deep sequencing to viral tagging: recent advances in viral metagenomics. Bioessays, 2013. 35(5): p. 436-42.

191. Ignacio-Espinoza, J.C., S.A. Solonenko, and M.B. Sullivan, The global virome: not as big as we thought? Curr Opin Virol, 2013. 3(5): p. 566-71.

192. von Mering, C., et al., Quantitative phylogenetic assessment of microbial communities in diverse environments. Science, 2007. 315(5815): p. 1126-30.

193. Li, L.L., et al., Bioprospecting metagenomes: glycosyl hydrolases for converting biomass. Biotechnol Biofuels, 2009. 2: p. 10.

194. Lorenz, P. and J. Eck, Metagenomics and industrial applications. Nat Rev Microbiol, 2005. 3(6): p. 510-6.

195. Perić-Concha, N. and P.F. Long, Mining the microbial metabolome: a new frontier for natural product lead discovery. Drug Discov Today, 2003. 8(23): p. 1078-84.

196. Greene, L.H., et al., The CATH domain structure database: new protocols and classification levels give a more comprehensive resource for exploring evolution. Nucleic Acids Res, 2007. 35(Database issue): p. D291-7.

197. Dessailly, B.H., et al., PSI-2: structural genomics to cover protein domain family space. Structure, 2009. 17(6): p. 869-81. 
198. Chubb, D., et al., Sequencing delivers diminishing returns for homology detection: implications for mapping the protein universe. Bioinformatics, 2010. 26(21): p. 2664-71.

199. Goldstein, R.A., The structure of protein evolution and the evolution of protein structure. Curr Opin Struct Biol, 2008. 18(2): p. 170-7.

200. Valas, R.E., S. Yang, and P.E. Bourne, Nothing about protein structure classification makes sense except in the light of evolution. Curr Opin Struct Biol, 2009. 19(3): p. 329-34.

201. Alquezar-Planas, D.E., et al., Discovery of a divergent HPIV4 from respiratory secretions using second and third generation metagenomic sequencing. Sci Rep, 2013. 3: p. 2468.

202. Bhat, A., et al., Isolation of cold-active, acidic endocellulase from Ladakh soil by functional metagenomics. Extremophiles, 2013. 17(2): p. 229-39.

203. Yang, C., et al., Characterization of a novel metagenome-derived 6-phospho- $\beta$ glucosidase from black liquor sediment. Appl Environ Microbiol, 2013. 79(7): p. 2121-7.

204. McDaniel, L.D., et al., Comparative metagenomics: natural populations of induced prophages demonstrate highly unique, lower diversity viral sequences. Environ Microbiol, 2013.

205. Roux, S., et al., Chimeric viruses blur the borders between the major groups of eukaryotic single-stranded DNA viruses. Nat Commun, 2013. 4: p. 2700.

206. Krupovic, M., Recombination between RNA viruses and plasmids might have played a central role in the origin and evolution of small DNA viruses. Bioessays, 2012. 34(10): p. 867-70.

207. Johnson, G.P., S.J. Goebel, and E. Paoletti, An update on the vaccinia virus genome. Virology, 1993. 196(2): p. 381-401.

208. Sekiguchi, J. and S. Shuman, Ligation of RNA-containing duplexes by vaccinia DNA ligase. Biochemistry, 1997. 36(29): p. 9073-9.

209. Finnegan, D.J., Retrotransposons. Curr Biol, 2012. 22(11): p. R432-7.

210. Delwart, E. and L. Li, Rapidly expanding genetic diversity and host range of the Circoviridae viral family and other Rep encoding small circular ssDNA genomes. Virus Res, 2012. 164(1-2): p. 114-21. 


\section{APPENDIX:}

\section{Chapter 3 Copyright Information}

Chapter 3 of this dissertation was previously published as a journal article in BioMed Central's Biology Direct open-access journal (http://www.biologydirect.com/content/7/1/13). The content and title of the journal article, "A novel virus genome discovered in an extreme environment suggests recombination between unrelated groups of RNA and DNA viruses", is one and the same as chapter 3 of this dissertation. Copyright on any research article in a journal published by BioMed Central is retained by the author(s), and is governed by the Creative Commons Attribution License 4.0 (http://creativecommons.org/licenses/by/4.0/legalcode). Authors are permitted to grant any third party the right to use the article freely as long as its integrity is maintained and its original authors, citation details and publisher are identified. Thus, permission is hereby granted by the authors, Geoffrey Diemer and Kenneth Stedman, to Portland State University to re-publish the journal article as chapter 3 of this dissertation. 